Journal of Patient-Centered

\title{
Abstracts From the 22nd Annual Health Care Systems Research Network Conference, April 13-16, 2016, Atlanta, Georgia
}

Follow this and additional works at: https://aah.org/jpcrr

Part of the Diseases Commons, Health and Medical Administration Commons, Health Information Technology Commons, Medical Education Commons, Medical Specialties Commons, and the Public Health Commons

\section{Recommended Citation}

Abstracts from the $22^{\text {nd }}$ annual Health Care Systems Research Network Conference, April 13-16, 2016, Atlanta, Georgia. J Patient Cent Res Rev. 2016;3:167-228. doi: 10.17294/2330-0698.1413

Published quarterly by Midwest-based health system Advocate Aurora Health and indexed in PubMed Central, the Journal of Patient-Centered Research and Reviews (JPCRR) is an open access, peer-reviewed medical journal focused on disseminating scholarly works devoted to improving patient-centered care practices, health outcomes, and the patient experience. 


\section{Abstracts From the $22^{\text {nd }}$ Annual Health Care Systems Research Network Conference, April 13-16, 2016, Atlanta, Georgia}

\section{AGING AND MULTIMORBIDITY}

B4-1:

Anesthesia Exposure and Risk of Dementia and Alzheimer's Disease: A Prospective Study

Erin J. Aiello Bowles, ${ }^{1}$ Eric B. Larson, ${ }^{1,2}$ Ryan P. Pong, ${ }^{3}$ Rod L. Walker, ${ }^{1}$ Melissa L. Anderson, ${ }^{1}$ Onchee $\mathrm{Yu},{ }^{1}$ Shelly L. Gray, ${ }^{4}$ Paul K. Crane, ${ }^{2}$ Sascha Dublin ${ }^{1,5}$

${ }^{1}$ Group Health Research Institute, Group Health Cooperative, Seattle, WA; ${ }^{2}$ Division of General Internal Medicine, Department of Medicine, University of Washington, Seattle, WA; ${ }^{3}$ Department of Anesthesiology, Virginia Mason Medical Center, Seattle, WA; ${ }^{4}$ School of Pharmacy, University of Washington, Seattle, WA; ${ }^{5}$ Department of Epidemiology, University of Washington, Seattle, WA

Background/Aims: Studies have demonstrated conflicting results about the association between anesthesia exposure and subsequent dementia risk. However, prior studies were retrospective, collecting data on anesthesia exposure after determining dementia status. We used prospectively collected data to evaluate the associations between anesthesia and risk of dementia or Alzheimer's disease (AD).

Methods: We conducted a cohort study among communitydwelling members of the Adult Changes in Thought cohort $(\mathrm{N}=3,988)$ who were age 65 or older and free of dementia at baseline. Participants self-reported all prior surgical procedures with general or neuraxial (spinal or epidural) anesthesia at baseline and reported new procedures every two years. We compared people who had high-risk surgery with general anesthesia, other surgery with general anesthesia, and other surgery with neuraxial anesthesia exposures to those who had no surgery and no anesthesia. We used Cox proportional hazards models to estimate hazard ratios (HR) and $95 \%$ confidence intervals $(\mathrm{CI})$ for dementia and $\mathrm{AD}$ associated with time-varying lifetime and recent (past 5 years) anesthesia exposures.

Results: At baseline, 254 (6\%) people reported never having anesthesia; $248(6 \%)$ had one or more high-risk surgeries with general anesthesia, 3,363 (84\%) had one or more other surgeries with general anesthesia, and $123(3 \%)$ had one or more surgeries with neuraxial anesthesia. Lifetime exposure of high-risk surgery with general anesthesia was not associated with an increased risk of dementia (HR: 0.86, 95\% CI: $0.58-1.28$ ) or AD (HR: $0.95,95 \%$ CI: $0.61-1.49)$ relative to no history of anesthesia. People with lifetime exposure of other surgery with general anesthesia had a lower risk of dementia (HR: $0.63,95 \%$ CI: 0.46-0.85) and AD (HR: 0.65, 95\% CI: 0.46-0.93) than people with no history of anesthesia. There was no association between recent anesthesia exposure and dementia or $\mathrm{AD}$. When comparing the results for highrisk surgery with general anesthesia directly to other surgery with general anesthesia, HR was 1.37 (95\% CI: 1.04-1.80) for dementia and 1.46 (95\% CI: 1.07-1.99) for AD.

Conclusion: Anesthesia exposure was not associated with an increased risk of dementia or AD in older adults. Future studies should continue to explore whether there is truly an increased risk of dementia and $\mathrm{AD}$ following high-risk surgery.

Keywords: anesthesia, dementia

A4-2:

Advance Care Planning Practices in Caring for Vulnerable Elders: An Analysis of Electronic Health Record Data and Interviews With Physicians

Ellis C. Dillon, ${ }^{1}$ Judith Chuang, ${ }^{1}$ Atul Gupta, ${ }^{2}$ Ming Tai-Seale ${ }^{1}$

${ }^{1}$ Palo Alto Medical Foundation Research Institute, Palo Alto, CA; ${ }^{2}$ Department of Economics, Stanford University, Stanford, CA

Background/Aims: Because patients with serious illnesses often reach a point when they cannot speak for themselves, it is critical to discuss advance care planning (ACP) and document patient preferences via advance health care directives (AHCD) and physician orders for life-sustaining treatment (POLST). We aim to characterize ACP discussion and documentation practices and determine how a palliative care program established in 2010 at the Palo Alto Medical Foundation influenced ACP practices.

Methods: We analyzed Palo Alto Medical Foundation patients $\geq 65$ years old who presented from 2013 to 2014 and had no record of ACP prior to 2013 in the electronic health record problem list. Logistic regression examined the relationship between ACP documentation and 1) type of serious illness (defined by the National Committee for Quality Assurance Palliative Care measurement set), 2) travel distance to palliative care specialists, and 3) patient and physician characteristics. We also interviewed physicians $(\mathrm{N}=15)$ in primary care, oncology, pulmonology and cardiology about ACP.

Results: Among 65,253 patients with no ACP prior to 2013, 11.6\% had ACP documentation by the end of 2014 and $88.4 \%$ did not. Among those with serious illness, at the end of 2 years $21.13 \%$ had ACP (12.09\% AHCD, 5.42\% POLST, $3.62 \%$ both) and $78.87 \%$ had no ACP. Patients 
with chronic obstructive pulmonary disease were more likely to have AHCD (odds ratio [OR]: 1.094, $\mathrm{P}<0.01$ ) and POLST (OR: 1.215, $\mathrm{P}<0.01$ ). Greater travel distance to palliative care lowered the likelihood of documented AHCD (OR: 0.904, $\mathrm{P}<0.01$ ) and POLST (OR: 0.866, $\mathrm{P}<0.01$ ). Male, Asian and black patients were less likely to have AHCD. Patients with brain cancer (OR: 4.165, $\mathrm{P}<0.01$ ), esophageal cancer (OR: 4.697, $\mathrm{P}<0.05)$ and debility (OR: 1.923, $\mathrm{P}<0.01)$ were more likely to have POLST. Some interviewed physicians believed ACP discussions were every physician's responsibility; others preferred appointing a "quarterback" (preferably a primary care physician). Interviewees suggested three stages of ACP: 1) initial patient-physician conversations, 2) patient investigates/ discusses with family, and 3) follow-up with physician.

Conclusion: The overwhelming majority of older patients without preexisting ACP do not have ACP documented at the end of a two-year period, even among patients with serious illnesses. Systematic efforts are needed to eliminate gender and racial disparities, integrate palliative care and train current health care teams to improve ACP.

Keywords: advance care planning, electronic health record

A4-3:

Patient-Reported Outcomes Collected as Part of the Medicare Annual Wellness Visit in the Health Care Systems Research Network

Heather Tabano, ${ }^{1}$ Thomas Gill, ${ }^{2}$ Kathryn Anzuoni, ${ }^{3}$ Heather Allore, ${ }^{2}$ Ann Gruber-Baldini, ${ }^{4}$ Sascha Dublin, ${ }^{5}$ Thomas Elliott, ${ }^{6}$ David Ganz, ${ }^{7}$ Ming Tai-Seale, ${ }^{8}$ Jerry Gurwitz, ${ }^{3}$ Elizabeth Bayliss ${ }^{1}$

${ }^{1}$ Institute for Health Research, Kaiser Permanente Colorado Denver, CO; ${ }^{2}$ Yale School of Medicine, New Haven, CT; ${ }^{3}$ Meyers Primary Care Institute, Worcester, MA; "University of Maryland School of Medicine, Baltimore, MD; ${ }^{5}$ Group Health Research Institute, Group Health Cooperative, Seattle, WA; ${ }^{6}$ HealthPartners Institute, Bloomington, MN; ${ }^{7}$ UCLA Department of Medicine, Los Angeles, CA; ${ }^{8} \mathrm{Pal}$ ( Alto Medical Foundation Research Institute, Palo Alto, CA

Background/Aims: For seniors with multiple chronic conditions, patient-reported outcomes (PROs) can provide meaningful information on patients' health, well-being and effects of treatment that transcend specific conditions. Effective use of PROs for clinical care and research requires an understanding of their availability and accessibility.

Methods: The data and measures workgroup of the HCSRNOAIC Advancing Geriatric Infrastructure and Network Growth (AGING) Initiative conducted an electronic survey to inventory PROs available at each HCSRN site and to understand their storage and accessibility. PRO domains inventoried included: self-rated health, functional status, mood, pain, cognition, fall risk and advance directives. For each domain, sites were asked to list specific measures used and to describe PRO data collection, storage and extraction. The inventory was distributed through the HCSRN governing board to representatives at each site.

Results: To date, 15 of 19 HCSRN sites have fully or partially completed the inventory. Four were unable to participate due to lack of PRO data or resources. The majority of sites rely on a combination of systematic outreach and patient initiation to complete the annual wellness visit (AWV) questionnaire, with the most complete data coming from the past 5 years. The percentage of Medicare patients completing an AWV questionnaire varies among sites, ranging from 5\% to $43 \%$. The most common method of storage is via "Smart Text" within text fields of the electronic health record. For the AWV, most sites address three or more of the PRO domains included in the inventory and many address additional domains as part of the AWV such as oral health, diet, exercise, incontinence, substance use and social needs.

Conclusion: Although there is some overlap, the method of collection, storage and availability of PROs gathered as part of the Medicare AWV vary widely across HCSRN sites. While there has been an improvement in the availability of this information in recent years, most sites' AWV questionnaire coverage remains low. The final inventory results will inform data infrastructure development and data collection processes across the HCSRN, and will facilitate engagement in multisite aging research related to multiple chronic conditions.

Keywords: aging, patient-reported outcomes

A4-4:

Multiple Chronic Conditions Associated With Hip Fracture Outcomes Among Males

Laurel A. Copelend, ${ }^{1}$ Charlene Quinn, ${ }^{2}$ Eileen M. Stock, ${ }^{1}$ Jinmyoung Cho, ${ }^{1}$ Rashmita Basu, ${ }^{1}$ John E. Zeber, ${ }^{1}$ Brian K. Ahmedani ${ }^{3}$

${ }^{1}$ Center for Applied Health Research, Baylor Scott \& White Health, Temple, TX; ${ }^{2}$ University of Maryland School of Medicine, Baltimore, MD; ${ }^{3}$ Center for Health Policy and Health Services Research, Henry Ford Health System, Detroit, MI

Background/Aims: Driven by an agenda to reduce hospital readmissions in integrated payer-provider systems, this study examined the association of multiple chronic conditions (MCC) with outcomes among hip fracture patients, including 30-day readmission and mortality. Models were developed in the Veterans Health Administration (VA) and will be applied to HCSRN sites in this collaboration among a Claude D. Pepper Older American Independence Center, two HCSRN sites and the VA.

Methods: The retrospective study used VA administrative data including the Corporate Data Warehouse, a snapshot of the VA's all-electronic medical records system updated nightly. Eligibility criteria were: age $>50$, male, hip fracture diagnosis (ICD-9 code 820) and inpatient hip fracture repair from October 2008 to September 2009. The primary independent variable of interest was number of chronic conditions per the Charlson and Elixhauser indices of comorbidity. Logistic regression modeled 30-day readmission, 1-year and 5-year 
mortality as a function of MCC, age, race/ethnicity, marriage status and VA Priority (i.e. 50-100\% disabled vs. other status). Odds ratio (OR) with $95 \%$ confidence interval (CI) estimated increased risk per MCC.

Results: The sample included 3,851 veterans with mean age of 75 (SD: 11, range: $50-100$ ); 18\% were minority race/ ethnicity, $23 \%$ were $50-100 \%$ disabled and $45 \%$ were married. The top five MCC were hypertension (67\%), diabetes (31\%), chronic obstructive pulmonary disease (29\%), deficiency anemia (25\%) and depression (24\%). MCC per patient averaged 4.1 (SD: 2.7, range: 0-15). Adverse outcomes 30-day readmission (16\%), 1-year mortality (31\%) and 5-year mortality $(65 \%)$ - were common. MCC were associated with greater risk of 30-day readmission (OR: 1.14, 95\% CI: 1.10 1.17), 1-year mortality (OR: 1.18, 95\% CI: 1.14-1.21) and 5-year mortality (OR: 1.27, 95\% CI: 1.23-1.31).

Conclusion: MCC greatly increased risk of adverse outcomes following hip fracture repair among men, irrespective of specific types of conditions. Each additional condition increased risk of readmission by about $14 \%$ so that risk would be doubled for a person with 7 disorders. Risk of 1-year mortality doubled at $5 \mathrm{MCC}$ and of 5-year mortality at 4 MCC. Supportive services for patients and families may need to incorporate information regarding risks for patients with MCC. Next steps include assessing the role of diabetes, dementia and pharmacotherapy in the relationship between MCC and hip fracture outcomes, and replicating the study among private not-for-profit patients in HCSRN.

Keywords: hip fractures, chronic disease

\section{PS1-18:}

\section{A Pilot Study of a Tailored Community Volunteer Intervention for Isolated Older Individuals}

\section{Andrew L. Avins, ${ }^{1}$ Andrea Altschuler ${ }^{1}$ \\ ${ }^{1}$ Division of Research, Kaiser Permanente Northern California, Oakland, CA}

Background/Aims: Socially isolated older individuals show greatly diminished quality of life, poorer outcomes with chronic medical conditions, greater and accelerated cognitive decline and a higher incidence of depression. Social isolation is also one of the strongest known predictors of mortality, with risks equivalent to or greater than well-known risk factors such as smoking, excessive alcohol consumption and obesity. Observational evidence suggests community volunteer work could help reduce these negative consequences, but a largescale intervention study of volunteer work tailored to the interests and values of each individual has not been conducted to date and the feasibility of conducting such a trial has never been assessed.

Methods: We conducted a small, prospective, single-cohort pilot study of community volunteer work tailored to each participant's interests in order to test its feasibility for use in a fully powered clinical trial. Eligible patients were $>60$ years old and scored $>40$ on the UCLA Loneliness Scale (Version 3). Participants were interviewed at baseline, and several relevant volunteer opportunities were identified (using volunteermatch.com and local resources) from which participants selected the most appealing opportunities. Study staff handled all arrangements for initiating the participant's volunteer work, and participants were asked to volunteer at least 4 hours per week. Participants were assessed after 2 and 4 months for social isolation, mood, self-esteem, quality of life and positive states of mind.

Results: Of 12 patients who enrolled in the study, 8 participated in the volunteer intervention. All participants had positive experiences, and almost all continued working in their volunteer positions after study closeout. Significant withinperson improvements were identified in UCLA Loneliness $(-5.1, \mathrm{P}<0.03)$ and the Rosenberg Self-Esteem Scale $(+3.4$, $\mathrm{P}<0.01)$ at 4 months; nonsignificant improvements were observed in all other measures. Qualitative data revealed universally positive experiences, with some participants expressing profoundly beneficial changes in quality of life.

Conclusion: A community volunteer intervention, tailored to each individual's interests and values, was found to be feasible for testing in a future clinical trial, and the intervention appears promising. Several important lessons were learned; recruitment and other challenges were noted, and methods for addressing these difficulties were identified.

Keywords: volunteerism, social isolation

PS1-19:

Multiple Chronic Conditions and Psychosocial Limitations in a Contemporary Cohort of Patients Hospitalized With an Acute Coronary Syndrome

Mayra Tisminetzky, ${ }^{1,2}$ Jerry Gurwitz,,2,3 David D. McManus, ${ }^{1,2,4}$ Jane S. Saczynski, ${ }^{1,2,3}$ Molly E. Waring, ${ }^{1}$ Nathaniel Erskine, ${ }^{1}$ Milena Anatchkova, ${ }^{1}$ David C. Parish, ${ }^{5}$ Darleen Lessard, ${ }^{1}$ Catarina Kiefe, ${ }^{1}$ Robert Goldberg ${ }^{1,2,4}$

${ }^{1}$ Department of Quantitative Health Sciences, University of Massachusetts Medical School, Worcester, MA; ${ }^{2}$ Meyers Primary Care Institute, Worcester, MA; ${ }^{3}$ Division of Geriatric Medicine, University of Massachusetts Medical School, Worcester, MA; ${ }^{4}$ Division of Cardiovascular Medicine, University of Massachusetts Medical School, Worcester, MA; ${ }^{5}$ Department of Community Medicine, Mercer University School of Medicine, Macon, GA

Background/Aims: As adults live longer, multiple chronic conditions have become more prevalent over the past several decades. We describe the prevalence of and patient characteristics associated with cardiac and noncardiac-related multimorbidities in patients discharged from the hospital after an acute coronary syndrome.

Methods: We studied 2,174 patients discharged from the hospital after an acute coronary syndrome at six medical centers in Massachusetts and Georgia between April 2011 and May 2013. Hospital medical records yielded clinical information, including presence of 8 cardiac-related and 8 
noncardiac-related morbidities on admission. We assessed multiple psychosocial characteristics during the index hospitalization using standardized in-person instruments.

Results: The mean age of the study sample was 61 years, $67 \%$ were men, and $81 \%$ were non-Hispanic whites. The most common cardiac-related morbidities were hypertension, hyperlipidemia and diabetes (76\%, 69\% and 31\%, respectively). Arthritis, chronic pulmonary disease and depression $(20 \%$, $18 \%$ and $13 \%$, respectively) were the most common noncardiac morbidities. Patients with 4 or more morbidities $(37 \%$ of the population) were slightly older and more likely to be women than those with 0-1 morbidity; they were also heavier and more likely to be cognitively impaired (26\% vs. $12 \%)$, have symptoms of moderate/severe depression (31\% vs. 15\%), have high perceived stress (48\% vs. 32\%), have a limited social network ( $22 \%$ vs. $15 \%$ ), have low health literacy (42\% vs. $31 \%$ ) and have low health numeracy (54\% vs. $42 \%)$.

Conclusion: Multimorbidity — highly prevalent in patients hospitalized with an acute coronary syndrome - is associated with psychosocial characteristics. This emphasizes the challenge of caring for these patients, which extends well beyond acute coronary syndrome management.

Keywords: multimorbidity, acute coronary syndrome

\section{PS-20: \\ Impact of Multimorbidity on Clinical Outcomes in Older Adults With Cardiovascular Disease: A Literature Review}

\author{
Mayra Tisminetzky, ${ }^{1}$ Robert Goldberg, ${ }^{1,3}$ Jerry $\mathrm{H}$. \\ Gurwitz $^{2}$
}

${ }^{1}$ Meyers Primary Care Institute, Worcester, MA; ${ }^{2}$ Department of Quantitative Health Sciences, University of Massachusetts, Worcester, MA; ${ }^{3}$ Division of Geriatric Medicine, University of Massachusetts, Worcester, MA

Background/Aims: We aimed to synthesize the current literature on the magnitude and impact of multiple chronic conditions on clinical outcomes, including total in-hospital and postdischarge mortality and hospitalizations, in older patients with cardiovascular disease.

Methods: A systematic review was conducted. Four electronic databases and article bibliographies were searched for publications from 2005 to 2015 that assessed the impact of multimorbidity on clinical outcomes in the elderly with cardiovascular disease. Identified studies were screened using predefined criteria for eligibility.

Results: Fifteen studies met our inclusion criteria. Multimorbidity was assessed by simply counting morbidities and/or by the Charlson and Elixhauser indices. Most the studies reported a significant association between number of morbidities or selected morbidities and the risk of dying; the most frequent conditions examined were diabetes, chronic kidney disease, anemia, chronic pulmonary disease and dementia/cognitive impairment.

Conclusion: There are limited data available on the magnitude and impact of multiple chronic conditions on clinical outcomes, including hospitalization and short- and long-term mortality, in patients with cardiovascular disease, and essentially no data on universal health outcomes (i.e. health-related quality of life, symptom burden and function). There is also a lack of consistency in the manner in which the burden of multimorbidity is assessed and characterized. Very few studies have addressed the true complexity of older patients with cardiovascular disease; how best to characterize multimorbidity in these patients and to relate this to clinical outcomes remains a substantial challenge.

Keywords: multimorbidity, cardiovascular disease

\section{PS-21: \\ New News From the Adult Changes in Thought Study: A Long-Standing Living Laboratory of Aging Funded for Five More Years}

Eric B. Larson, ${ }^{1}$ Erin J. Aiello Bowles, ${ }^{1}$ Rod L. Walker, ${ }^{1}$ Melissa L. Anderson, ${ }^{1}$ Darlene White, ${ }^{1}$ KatieRose Richmire, ${ }^{1}$ William W. Lee, ${ }^{1}$ Steven L. Balch, ${ }^{1}$ Andrea Z. LaCroix, ${ }^{2}$ Dori Rosenberg, ${ }^{1}$ Sascha Dublin, ${ }^{1}$ Paul K. Crane $^{3}$

${ }^{1}$ Group Health Research Institute, Group Health Cooperative, Seattle, WA; ${ }^{2}$ Department of Family Medicine and Public Health, University of CaliforniaSan Diego, San Diego, CA; ${ }^{3}$ Department of Medicine, University of Washington, Seattle, WA

Background/Aims: Delivery system-based research in enumerated populations provides a unique opportunity to study complex chronic diseases as they develop. Studies of disorders that affect people late in life can be particularly valuable. The Adult Changes in Thought (ACT) study was recently awarded five more years of funding. This makes ACT one of the longest continually funded studies and establishes it as a preeminent living laboratory for aging research.

Methods: Originally a joint project between Group Health and the University of Washington, the ACT study recruited a cohort of randomly selected Group Health enrollees over age 65 without dementia from 1994 to 1996 . Initially 2,581 persons were recruited, but in response to cohort shrinkage over time, we developed expansion and ongoing replacement strategies to maintain a currently active cohort of approximately 2,000 living persons who are followed every 2 years. The original outcomes of interest in ACT were Alzheimer's disease and related dementias based on standardized diagnostic methods supplemented by neuropathologic outcomes. This funding cycle we've added a new outcome "resilience" to specifically study persons who defy expectations by avoiding cognitive decline and frailty well into late life.

Results: ACT has enrolled over 5,000 subjects to date and includes over 1,000 cases of incident dementia (over $70 \%$ Alzheimer's disease) and over 600 autopsy cases, of which about half have extensive frozen tissues from a rapid autopsy protocol. The laboratory now includes a biobank with a vast array of genomic information along with a population-based neuropathology biobank that is unique worldwide. The new 
funding cycle will include state-of-the-art measures of physical activity (e.g. accelerometers, inclinometers) to determine how physical activity and sedentary time affect outcomes, especially resilience, and also include unique neuropathologic markers (synaptosomes and histelide) to better understand brain aging. ACT has always supported other investigations, and we are well equipped to support HCSRN scientists who wish to use our living learning laboratory of aging.

Conclusion: The long-running ACT study in the new funding cycle will focus on the science of aging and multimorbidity, understanding resiliency and robust aging, and sharing data from our extensive data repository for other studies of interest. Keywords: aging, resiliency

PS-22:

A Systematic Review of Conceptual Frameworks of
Medical Complexity and New Model Development

Leah Zullig, ${ }^{1}$ Heather E. Whitson, ${ }^{2}$ S. Nicole Hastings, ${ }^{1}$

Christopher Beadles, ${ }^{3}$ Julia Krauchanka, ${ }^{4}$ Igor Akushevich, ${ }^{5}$ Matthew Maciejewski ${ }^{1}$

${ }^{1}$ Health Services Research and Development, Durham VA Medical Center, Duke University Medical Center, Durham, NC; ${ }^{2} \mathrm{HCSRN}-\mathrm{OAIC}$ AGING Initiative, Durham VA Medical Center, Duke University Medical Center, Durham, NC; ${ }^{3}$ Health Services Research, University of North Carolina at Chapel Hill, Chapel Hill, NC; ${ }^{4}$ Duke University, Durham, NC; ${ }^{5}$ Center for Population Health, Duke University, Durham, NC

Background/Aims: Patient complexity entails multimorbidity as well as interacting contextual factors that affect patient risk for adverse outcomes. Our objective was to develop a conceptual model of factors relating to patients' medical complexity by addressing gaps identified in a review of published conceptual models.

Methods: We searched for English-language MEDLINE papers published between Jan. 1, 2004, and Jan. 16, 2014. Two reviewers independently evaluated abstracts. All authors contributed to the development of the conceptual model in an iterative process.

Results: From 1,606 identified abstracts, six conceptual models were selected. One additional model was identified through reference review. These models included works by Capobianco and Lio (2013), Giovannetti et al. (2013), Grembowski et al. (2014), Holzhausen et al. (2011), Piette and Kerr (2006), Safford et al. (2007) and Shippee et al. (2010). Each model had strengths, but several constructs were not fully considered: 1) contextual factors such as interpersonal, organizational or community relations; 2) dynamics of complexity as a condition that fluctuates over time; 3) patient preferences; 4) acute health shocks and medical events that influence health; and 5) resilience in the face of such stressors. To build on prior literature while addressing these gaps, we developed a new model, the "Cycle of Complexity." This model illustrates relationships between acute shocks and health events, health care access and utilization, patient workload and capacity, and patient preferences in the context of interpersonal, organizational and community factors. It can be used to understand within-person changes over time as well as between-person differences at a single point in time. The Cycle of Complexity can be applied to evaluation of multiple outcomes and acknowledges possible health decline as well as resilience.

Conclusion: The Cycle of Complexity model may inform studies on the etiology of and changes in patient complexity, the relationship between complexity and patient outcomes, and intervention development to improve modifiable elements of care for complex patients. The model may be helpful in applying big data resources to address research questions relevant to medically complex populations.

Keywords: multimorbidity, resilience

\section{CANCER}

B3-1:

Survival After Recurrence of Stage I-III Breast, Colorectal, and Lung Cancer

Michael J. Hassett, ${ }^{1}$ Hajime Uno, ${ }^{2}$ Angel M. Cronin, ${ }^{1}$ Nikki M. Carroll, ${ }^{3}$ Mark C. Hornbrook, ${ }^{4}$ Debra Ritzwoller ${ }^{3}$

${ }^{1}$ Dana-Farber Cancer Institute, Boston, MA; ${ }^{2}$ Harvard Medical School, Boston, MA; 'Institute for Health Research, Kaiser Permanente Colorado, Denver, CO; ${ }^{4}$ Center for Health Research, Kaiser Permanente Northwest, Portland, OR

Background/Aims: Cancer recurrence is common, costly and lethal. Little is known about it in community-based populations, in part because electronic health records and tumor registries usually lack recurrence status. We examined determinants of survival duration after recurrence of breast, colorectal and lung cancers using two databases with excellent recurrence and death data.

Methods: Recurrence data were abstracted from medical records in the Cancer Care Outcomes Research and Surveillance (CanCORS) study and by two Cancer Research Network (CRN) sites - Kaiser Permanente Colorado and Kaiser Permanente Northwest. Death data were extracted from death certificates collected from the National Death Index or health plans. Claims and utilization data provided information on comorbidities and treatments. CanCORS enrolled only those patients who consented, whereas the HCSRN-CRN sample included all eligible patients who received care. This analysis included 1,653 patients who developed recurrence after having had definitive therapy for stage I-III cancer and were followed for at least one day after recurrence.

Results: Restricted mean survival times (RMST) after recurrence for breast, colorectal and lung cancer patients were 34.0, 27.7 and 16.9 months, respectively. Multivariable models revealed lower RMST in breast cancer was associated with: age $\geq 70$ (vs. $<55)$, higher comorbidity burden $(2+$ vs. 0 ), stage III (vs. stage 1), chemotherapy for primary tumor (vs. no chemotherapy), and distant/regional recurrence vs. 
local. Higher RMST in breast cancer was associated with: longer time to recurrence, and stage III and radiation for primary tumor (vs. stage I or no radiation for primary tumor). Lower RMST in colorectal cancer was associated with: age $\geq$ 70 (vs. < 55), comorbidity burden $\geq 1$ (vs. 0), distant/regional recurrence (vs. local), and radiation therapy for recurrence (vs. no radiation). Higher RMST in colorectal cancer was associated with: chemotherapy for recurrence but not for primary (vs. no chemotherapy for primary or recurrence). Higher RMST in lung cancer was associated with: income $\geq$ $\$ 60,000$ (vs. $<\$ 20,000$ ). Lower RMST in lung cancer was associated with: stage III (vs. stage I), and interaction of income $\geq \$ 60,000$ and comorbidity burden $\geq 2$ (vs. income $<$ $\$ 60,000$ or comorbidity of 0 ).

Conclusion: Breast and colorectal, but not lung, cancer patients with local recurrences had longer survival than those with regional/distant recurrences. Shorter time to recurrence was associated with inferior survival after recurrence among breast cancer patients only. Original cancer stage was a predictor of survival after recurrence; original cancer grade was not.

Keywords: cancer recurrence, cancer outcomes

\section{B3-2:}

\section{Development of Data Infrastructure to Monitor Lung Cancer Screening Use and Outcomes in Four Cancer Research Network Sites}

V. Paul Doria-Rose, ${ }^{1}$ Lori C. Sadoka, ${ }^{2}$ Christine M. Neslund-Dudas, ${ }^{3}$ Debra P. Ritzwoller, ${ }^{4}$ Heather S. Feigelson, ${ }^{4}$ Michael J. Simoff, ${ }^{5}$ Lawrence H. Kushi, ${ }^{2}$ Michael K. Gould 6

${ }^{1}$ Healthcare Delivery Research Program, National Cancer Institute, Bethesda, MD; '2Division of Research, Kaiser Permanente Northern California, Oakland, CA; ${ }^{3}$ Department of Public Health Sciences, Henry Ford Health System, Detroit, MI; ${ }^{4}$ Institute for Health Research, Kaiser Permanente Colorado, Denver, CO; ${ }^{5}$ Henry Ford Hospital, Henry Ford Health System, Detroit, MI; ${ }^{6}$ Department of Research \& Evaluation, Kaiser Permanente Southern California, Pasadena, CA

Background/Aims: With the National Lung Screening Trial finding a $20 \%$ reduction in lung cancer mortality among individuals screened by low-dose computed tomography (LDCT) versus chest radiography, and the subsequent U.S. Preventive Services Task Force (USPSTF) grade B recommendation, lung cancer screening is reemerging as a priority area in cancer prevention research. Questions remain, however, as to whether the ratio of benefits to harm observed in the National Lung Screening Trial will differ in routine clinical practice. Furthermore, in its National Coverage Determination, the Centers for Medicare \& Medicaid Services (CMS) required that all facilities performing LDCT submit screening data to an approved registry. To facilitate research, quality improvement and compliance with CMS requirements, there is a need to create data systems to monitor LDCT screening.

Methods: The Cancer Research Network (CRN) provides infrastructure support to promote cancer research within integrated health care systems. Four CRN sites received pilot funding from the National Cancer Institute starting in December 2014 to develop capacity to collect data regarding several aspects of LDCT screening: eligibility (e.g. smoking status), exam indication (e.g. screening or surveillance), exam results (Lung-RADS category, nodule characteristics), and early outcomes (lung cancer detection, follow-up diagnostic testing).

Results: The four sites include the Henry Ford Health System and three Kaiser Permanente regions (Colorado, Northern California and Southern California). All sites use USPSTF recommendations (or slight variations) for screening eligibility; collectively, approximately 150,000 health plan members within these systems are eligible for screening. Implementation of screening ranged from a small pilot program starting at two medical centers to more broad availability at the other sites, with varying degrees of outreach to promote screening. Strategies to collect information on LDCT include referral templates that ascertain eligibility and exam indication, use of hashtags for extraction of LungRADS category from radiology reports, retrieval of structured data from electronic health records and natural language processing to ascertain nodule characteristics.

Conclusion: Screening registry data collection is often laborintensive, requiring manual abstraction of medical record information. The development of strategies and tools to efficiently extract LDCT screening data from medical record systems will facilitate research to assess the utilization and outcomes of lung cancer screening as it is implemented in community settings.

Keywords: lung cancer, screening

B3-3: Feasibility of Establishing a Cohort in the Setting of
U.S. Health Care Systems

Amanda Black, ${ }^{1}$ Amy Berrington de Gonzalez, ${ }^{1}$ Robert Hoover, ${ }^{1}$ LeeAnn M. Rohm, ${ }^{2}$ Andrew Sterrett, ${ }^{2}$ Nicolas Wentzensen, ${ }^{1}$ Michelle Wrenn, ${ }^{2}$ Hannah P. Yang, ${ }^{1}$ Heather Spencer Feigelson ${ }^{2}$

${ }^{1}$ Division of Cancer Epidemiology and Genetics, National Cancer Institute, Bethesda, MD; 'Institute for Health Research, Kaiser Permanente Colorado, Denver, CO

Background/Aims: Epidemiologic cohorts have contributed substantially to our understanding of potential risk factors and biological markers for cancer and other chronic diseases. Integrated health care systems offer a promising setting to establish a new large-scale prospective biospecimen-based cohort for research on disease etiology and outcomes. Health care systems with electronic medical records provide some unique advantages for establishing the next generation of cohort studies. Our objective in this Kaiser Permanente Colorado (KPCO) pilot study was to assess the feasibility of leveraging existing health care system infrastructure to establish a prospective cohort.

Methods: In 2014, a total of 1,350 eligible KPCO members 
(aged 40-74 years, cancer-free, KPCO member for at least 12 months) were invited to participate, 850 via email and 500 via mail. Participants were asked to provide a blood sample and to complete a self-report risk factor survey. An interactive voice response system was used for participant reminders.

Results: Of those randomized to receive an email invitation to participate, $630(74 \%)$ had a valid email address; $98 \%(n=490)$ in the mail arm had a valid mailing address for a total of 1,120 contactable members. Overall, 115 individuals consented to participate, with greater recruitment success in the email arm $(12 \%)$ than in the mail arm $(8 \%)$. Of those who consented, $86(75 \%)$ provided a blood sample and $94(82 \%)$ completed and returned the survey by mail. In both arms, more females than males consented, with the highest consent rate among 60-69-year-olds and the lowest among 40-49-year-olds. The majority of participants in both arms were non-Hispanic white. Compared to the mail arm, there was a greater consent rate among women (68\% vs. 57\%), African-Americans (5\% vs. $3 \%$ ) and Hispanics (22\% vs. $17 \%$ ) in the email arm. There was a lower consent rate in the email arm among members over 70 years old ( $9 \%$ vs. $20 \%$ ).

Conclusion: The success of this study demonstrates the feasibility of implementing a biospecimen-based cohort in a health care system setting. The study also served to highlight potential problems as well as solutions to these issues.

Keywords: feasibility study, cancer

\section{B3-4:}

\section{Rapid Case Ascertainment - Two Algorithms to Identify Newly Diagnosed Cancer}

Christina L. Clarke, ${ }^{1}$ Sheila Weinmann, ${ }^{2}$ Stephen K. Van Den Eeden, ${ }^{3}$ Don Bachman, ${ }^{2}$ Jun Shan, ${ }^{3}$ Michelle Wrenn, ${ }^{1}$ Heather S. Feigelson ${ }^{1}$

${ }^{1}$ Institute for Health Research, Kaiser Permanente Colorado, Denver, CO; ${ }^{2}$ Center for Health Research, Kaiser Permanente Northwest, Portland, OR; ${ }^{3}$ Division of Research, Kaiser Permanente Northern California, Oakland, CA

Background/Aims: The Kaiser Permanente Research Bank is a biobanking effort that includes a general cohort, a pregnancy cohort and a cancer cohort. A priority of the cancer cohort is rapid case identification (RCA) of newly diagnosed members. Because of the large volume of cancer cases diagnosed each year across Kaiser Permanente, this method must be automated and utilize electronic files; manual review of records is not feasible. We developed two algorithms using pathology reports that could identify most newly diagnosed cancers within days of diagnosis.

Methods: We developed two RCA algorithms; one utilized systematized nomenclature of medicine (SNOMED) codes and one used natural language processing (NLP) to read directly from the pathology report. All available pathology reports with SNOMED coding for 2013 were analyzed with SAS software at Kaiser Permanente Northwest (KPNW), Colorado (KPCO) and Northern California (KPNC), and all available 2013 pathology reports from KPCO were analyzed for NLP and processed using Linguamatics I2E. Cancer cases that were flagged using SNOMED or NLP were compared to the 2013 Virtual Tumor Registry (VTR, the gold standard) at each participating site to obtain sensitivity and specificity of the algorithm. We analyzed the data against all reportable cancers as well as against those that were diagnosed within a Kaiser Permanente facility and through pathology (limited VTR).

Results: Using the full VTR, the sensitivity for the SNOMED algorithm was $54 \%, 60 \%$ and $85 \%$ for KPCO, KPNW and KPNC, respectively. Specificity was $94 \%$, 94\% and $90 \%$, respectively. When using the limited VTR, the sensitivity increased to $75 \%, 74 \%$ and $94 \%$, respectively. The NLP algorithm at KPCO resulted in a higher sensitivity (58\% full VTR, 80\% limited VTR) than the SNOMED algorithm but a lower specificity (89\%). Importantly, the RCA methods capture a wide range of cancer types across all stages.

Conclusion: Our two RCA algorithms are able to capture up to $80 \%$ of all cancer cases diagnosed while minimizing the number of people falsely identified as having cancer. These methods should help us minimize survival bias in the cancer cohort and possibly allow us to collect pretreatment blood samples on a portion of our cohort members.

Keywords: algorithm, cancer

\section{PS2-41: \\ Process-Related Quality Measures of Early- and Late-Stage Laryngeal Cancer Care}

\section{Steven Chang ${ }^{1}$}

\section{${ }^{1}$ Department of Public Health Sciences, Henry Ford Health System, Detroit, MI}

Background/Aims: The purpose of this study was to evaluate the quality measures established by the American Head and Neck Society (AHNS) quality committee. The measures were intended to assess quality care in treating laryngeal cancer beyond the traditional measures of survival and locoregional control. This study also attempted to identify specific measures from within AHNS guidelines that may relate to locoregional control and/or survival.

Methods: Retrospective review of patients with laryngeal cancer at a tertiary, academic center between 1991 and 2012. Patients were divided into early stage and late stage based on American Joint Committee on Cancer staging and analyzed separately. The major measures of quality, including overall survival, proper staging of the tumor prior to initiation of treatment, documentation of adverse pathology features (perineural invasion, lymphovascular invasion, positive margin, extracapsular spread), appropriate referral for adjuvant therapy (radiation and/or chemotherapy) and proper adherence to follow-up per National Comprehensive Cancer Network guidelines, were examined. The influence of these measures on survival also was analyzed.

Results: A total of 806 cases of laryngeal cancer were identified. Of those, $76.9 \%$ had proper documentation of tumor-node-metastasis staging. Of the 602 staged, 56\% were 
early stage and 44\% were late stage, $55 \%$ had documentation of adverse pathology, $52 \%$ were given appropriate referral for radiation therapy and $70 \%$ had appropriate follow-up. There was a statistically significant difference in survival for those patients who received appropriate initial staging when compared to nonstaged cases. This significance persisted when controlling for early- versus late-stage cases.

Conclusion: This study supports the validity of processrelated quality measures from the AHNS. This is the first study of head and neck cancers suggesting that processrelated measures can influence outcome measures such as overall survival.

Keywords: head and neck cancer, outcomes and quality

\section{PS2-42:}

\section{Multiple Myeloma Data in the Cancer Research Network}

\section{Rebecca A. Ziebell, ${ }^{1}$ Jessica Chubak, ${ }^{1}$ Mara M. Epstein ${ }^{2}$ \\ ${ }^{1}$ Group Health Research Institute, Group Health Cooperative, Seattle, WA; ${ }^{2}$ Meyers Primary Care Institute, Worcester, MA}

Background/Aims: Multiple myeloma is a rare plasma cell cancer that disproportionately affects men and blacks/ African-Americans. Research supported by initiatives such as the Cancer Research Network (CRN) is needed to advance knowledge about this uncommon but increasingly prevalent disease. The CRN is a consortium funded by the National Cancer Institute to support cancer research among a subset of HCSRN sites. The data collected and maintained by CRN sites facilitate a variety of multiple myeloma research opportunities.

Methods: Data from eight funded and three affiliate CRN sites were included in this analysis. Counts and characteristics of multiple myeloma diagnoses during 2003-2012 were obtained from the CRN Cancer Counter, an informatics tool that provides aggregate data to assist in study planning. Sitespecific descriptive analyses included annual counts of incident cases as well as demographic distributions. Updated counts, enrollee retention metrics and cancer treatment information will be obtained from each site's Virtual Data Warehouse in early 2016 via a centrally developed SAS software program.

Results: During 2003-2012, 5,137 multiple myeloma cases were diagnosed among $11 \mathrm{CRN}$ sites. Annual diagnoses increased steadily from 405 cases in 2003 to 610 in 2012. The majority of diagnoses $(61 \% ; n=3,117)$ occurred among persons age 65 or older, although that percentage ranged from $37 \%$ to $70 \%$ across sites. Males accounted for $57 \%(n=2,920)$ of diagnoses with a range of $49-63 \%$ across sites. Blacks/ African-Americans comprised $13 \%(n=686)$ of total diagnoses, although two sites contributed $>80 \%$ of those cases. Among persons with known ethnicity, Hispanics accounted for $7 \%$ $(n=289)$ of diagnoses, with one site contributing nearly $80 \%$ of those cases.

Conclusion: The CRN supports the infrastructure to maintain high-quality data (from tumor registries as well as internal health plan systems) on patients diagnosed with cancer at participating sites. Furthermore, the multisite CRN environment allows for the study of rare cancers among a large, demographically diverse patient population. Thus, the CRN provides a setting that is well suited for research in what is potentially one of the largest multiple myeloma cohorts with longitudinal data.

Keywords: multiple myeloma, cancer

\section{PS-43: \\ Determinants of Patient Choice of Health Care Providers for Breast Cancer Treatment}

Ashish Rai, ${ }^{1}$ Caroline A. Thompson, ${ }^{2}$ Allison W. Kurian, ${ }^{3}$ Harold S. Luft ${ }^{1}$

${ }^{1}$ Palo Alto Medical Foundation for Health Care, Research and Education, Palo Alto Medical Foundation Research Institute, Palo Alto, CA; ${ }^{2}$ Division of Epidemiology \& Biostatistics, Graduate School of Public Health, San Diego State University, San Diego, CA; ${ }^{3}$ Department of Medical Oncology, Stanford University School of Medicine, Stanford, CA

Background/Aims: Unless they are constrained by their insurance coverage, patients routinely seen within an integrated delivery system (IDS) have choices as to where they will be treated. If the IDS provides a reasonably broad range of cancer services, a major alternative will be an academic medical center (AMC). We hypothesize that among breast cancer patients initially diagnosed in a communitybased IDS, the likelihood of seeking cancer-related care in a nearby AMC will be related to: a) cancer severity, b) other comorbidities, c) geographic proximity to the AMC vs. the IDS - likely more important for interventions requiring frequent visits (e.g. chemo vs. surgery), d) sociodemographic factors, e) "connectedness" of the patient to the IDS, and f) enrollment in a capitated plan.

Methods: The Oncoshare database includes all patients diagnosed with or treated for breast cancer between 2000 and 2013 at Palo Alto Medical Foundation (PAMF), a community IDS, or Stanford University Medical Center, a neighboring AMC. The database links electronic health records from both institutions with data from the California Cancer Registry. We will examine treatment venues for patients initially diagnosed at PAMF using electronic health records data to determine baseline health, comorbidities and breast cancer treatments and California Cancer Registry data for tumor characteristics, sociodemographic factors (based on census tract) and primary payer at diagnosis. Patient "connectedness" is measured by duration with the primary care provider and number of other specialists seen at PAMF. We will use multiple variable logistic regression models to examine determinants of choice of IDS vs. AMC providers for breast cancer treatment.

Results: During the studied period, 3,784 women were newly diagnosed at PAMF; $80 \%$ received all their treatment at $\mathrm{PAMF}, 5 \%$ received all their postdiagnostic care at the $\mathrm{AMC}$, and 4\% received treatment at both sites during their initial cancer episode. Treatment sites were unknown for $11 \%$ 
of patients. Analyses to understand these patterns of where patients seek care are underway.

Conclusion: Observational studies often contrast resource use and outcomes across delivery systems. If patients have choices among systems, however, it is critical to understand whether their reasons for seeking care at different sites may partially account for observed differences in performance.

Keywords: patterns of care, breast cancer

PS-44:

Restructuring Oncology Payments: Estimates of Resource Use by Phases of Oncology Care in Breast Cancer Patients

Ashish Rai, ${ }^{1}$ Su-Ying Liang, ${ }^{1}$ Caroline A. Thompson, ${ }^{2}$ Harold S. Luft ${ }^{1}$

${ }^{1}$ Palo Alto Medical Foundation for Health Care, Research and Education, Palo Alto Medical Foundation Research Institute, Palo Alto, CA; '2Division of Epidemiology \& Biostatistics, Graduate School of Public Health, San Diego State University, San Diego, CA

Background/Aims: Under the fee-for-service system, oncologists do not get paid for many high-value and potentially cost-saving services, but may generate revenue from markups on certain chemotherapy drugs. The inappropriate incentives were at the core of an alternative payment model proposed by American Society of Clinical Oncology (ASCO). ASCO proposed new payment groups to support vital diagnostic, treatment and care management services not compensated under the current system. The aims are to: a) estimate the cost of oncology care by types of services for each payment group proposed by ASCO; b) estimate time and resource use for each payment group; and c) assess whether cost, resource use and clinician time varies by clinical measures.

Methods: We will use electronic health records (EHR) data to identify breast cancer patients diagnosed or treated from 2012 to 2014 at a large health care system in Northern California. Billings will be assigned to payment groups (e.g. new patient, treatment month, transition month, monitoring month) following ASCO's proposed algorithm. Costs will be stratified by service types (e.g. infusion, drug expenses, lab tests) and payment groups. We will use EHR access log data to estimate clinician time by service or activity type (e.g. treatment planning, patient education, etc.) within each payment group. Comorbidities and treatment regimens will be ascertained from patient encounter data for relevant diagnosis codes. Natural language processing algorithms will be used to extract patient performance status (Eastern Cooperative Oncology Group or Karnofsky functional status). Drug toxicity and drug administration factors will be calculated according to the Moffitt scale and the Michiana Hematology Oncology patient acuity scale. Average cost, time and resource use from aims (a) and (b) will be adjusted by a composite factor comprising of comorbidity score, performance status, drug toxicity factor and drug administration factor.

Results: We have obtained IRB approval and are extracting
EHR data for the study. Based on past annual averages, we expect to have data on approximately 2,500 breast cancer patients diagnosed or treated at the health care system.

Conclusion: This study will provide preliminary but critical evidence needed for consideration and implementation of ASCO's payment model.

Keywords: payment reform, episodes of care

PS-45:

Health Care Utilization and Cancer Incidence Following Solid Organ Transplant

Nirupa R. Ghai, ${ }^{1}$ Steven J. Jacobsen, ${ }^{1}$ Michael J. Silverberg, ${ }^{2}$ Neil M. Kogut, ${ }^{3}$ MaLou A. Valencia, ${ }^{4}$ ChuLing Yu, ${ }^{5}$ Virginia P. Quinn ${ }^{1}$

${ }^{1}$ Department of Research \& Evaluation, Kaiser Permanente Southern California, Pasadena, CA; ${ }^{2}$ Division of Research, Kaiser Permanente Northern California, Oakland, CA; ${ }^{3}$ Kaiser Permanente Southern California, Pasadena, CA; ${ }^{4}$ National Transplant Service, Kaiser Permanente Southern California, Los Angeles, CA; ${ }^{5}$ Mid-Atlantic Permanente Research Institute, Kaiser Permanente Mid-Atlantic States, Rockville, MD

Background/Aims: Population-based registry studies have found increases in lung, kidney, skin and thyroid cancers among organ transplant recipients compared with the general population. These studies link data from national transplant services and state cancer registries, thereby limiting the ability to describe recommended health care utilization, including preventive services (e.g. influenza vaccinations) and outpatient visits (9 within transplant year), and incident diagnoses of hypertension and diabetes (estimated to be $50 \%$ and $18 \%$, respectively). The goal of this study was to characterize health care utilization and cancer incidence among solid organ transplant recipients in Kaiser Permanente Southern California (KPSC).

Methods: KPSC transplant registry data was linked to electronic medical records on solid organ transplants from 1990 to 2014. Data was stratified by kidney, liver, heart and lung transplants.

Results: Among 4,336 transplant recipients, 62\% (2,703/4,336) were white, $80 \%(3,621 / 4,336)$ were $>35$ years old, $27 \%$ $(1,158 / 4,336)$ were past smokers and the mean membership length was 5.6 years. Past smoking was highest for lung transplant recipients $(34 \%, 63 / 185)$ and liver transplant recipients $(33 \%$, $418 / 1,271$ ). Survival among all transplant recipients was $89 \%$ at 2 years posttransplant yet decreased to $65 \%$ at 10 years, with the lowest survival among lung recipients (35\% at 10 years). Within the first year posttransplant, recipients had a mean number of 5 primary care visits, 7 nephrology visits and 2 visits each for dermatology, urology, and obstetrics and gynecology (women). Influenza vaccination rates have increased over time and were as high as $96 \%$ among lung recipients during the $2014-$ 2015 influenza season. Roughly, 12\% $(322 / 2,601)$ and 14\% $(151 / 1,082)$ of transplant recipients had an incident diagnosis of hypertension and diabetes. Finally, there was increased risk of 
all cancers excluding nonmelanoma skin cancer (standardized incidence ratio: $2.15,95 \%$ confidence interval: $1.93-2.39$ ), with the largest incidence for non-Hodgkin's lymphoma, lung cancer and kidney cancer.

Conclusion: Transplant recipients in KPSC met the recommended number of outpatient visits, including specialty care visits, within the first year posttransplant. Incidences of hypertension and diabetes were lower than expected, and influenza vaccination rates were high. There was a twofold increase in cancer incidence among the solid organ transplant population.

Keywords: transplant, cancer incidence

\section{ECl Awardee:}

The Impact of Clinical Guidelines on Prostate Cancer Screening Practices in a Population-Based Setting, 2000-2013

Mara M. Epstein, ${ }^{1}$ Daniel M. Frendl, ${ }^{2}$ Hassan Fouayzi, ${ }^{1}$ Richard Krajenta, ${ }^{3}$ Benjamin A. Rybicki, ${ }^{3}$ Mitchell $\mathrm{H}$. Sokoloff ${ }^{4}$

${ }^{1}$ Meyers Primary Care Institute, Worcester, MA; ${ }^{2}$ Department of Quantitative Health Sciences, University of Massachusetts Medical School, Worcester, MA; ${ }^{3}$ Department of Public Health Sciences, Henry Ford Health System, Detroit, MI; ${ }^{4}$ Department of Urology, University of Massachusetts Medical School, Worcester, MA

Background/Aims: Controversial clinical guidelines have recommended against widespread prostate-specific antigen (PSA) testing, despite evidence suggesting a benefit in certain populations. This study evaluates the impact of the publication of prostate cancer screening trial results in 2009 and changes to the U.S. Preventive Services Task Force (USPSTF) guidelines in 2012 on temporal trends in the use of PSA tests among men 40-80 years old, with follow-up through 2013.

Methods: Men aged 40-80 without a history of prostate cancer who sought care at Fallon Health (Meyers Primary Care Institute [MPCI]) or Henry Ford Health System (HFHS) contributed to this analysis. We counted one PSA test per person per calendar year, with rates defined per 1,000 personyears. Men were censored based on prostate cancer diagnosis, PSA test results $\geq 4 \mathrm{ng} / \mathrm{mL}$, disenrollment, death or end of follow-up (Dec. 31, 2014). We also examined trends in PSA testing among high-risk men (African-American, family history of prostate cancer). Test rates were compared among three time periods: 2000-2008, 2009-2012 and 2013-2014.

Results: On average, 17,144 men at MPCI and 16,733 men at HFHS had a PSA test each year. Mean age at PSA test was 57 years, which increased over time at both sites. PSA test rates at MPCI declined 33\% over the study period from an average of 474 tests/1,000 person-years (2000-2008) to $391 / 1,000$ person-years (2009-2012) to $317 / 1,000$ personyears (2013). At HFHS, PSA test rates rose gradually from 2000 to 2008 , with between $30 \%$ and $52 \%$ of the eligible population $(20,838$ of 69,550 men in 2000 and 19,366 of 37,434 men in 2008) undergoing $\geq 1$ test, followed by a $21 \%$ decline from 2008 to 2013 (7,151 of 23,749 men in 2013). At both sites, this decline was attenuated among high-risk men. Conclusion: This analysis of two population-based electronic health datasets provides evidence of decreasing use of PSA testing over time, although high-risk populations experienced a lesser decline. Although we are unable to determine causality, it is plausible that results of recent screening trials and/or restrictive changes to the USPSTF guidelines have impacted PSA testing practices over the past 14 years. As a next step, we will investigate trends in medical follow-up to elevated PSA test results.

Keywords: prostate cancer, screening

\section{CARE IMPROVEMENT}

B4-1:

Impact of a Medically Complex Patient Care Model on Cost and Utilization Among Adolescents and Young Adults With Special Health Care Needs

\author{
Daniel D. Maeng, ${ }^{1}$ Susan R. Snyder, ${ }^{1}$ Thomas W. Davis ${ }^{2}$
}

${ }^{1}$ Center for Health Research, Geisinger Health System, Danville, PA; '2Department of Internal Medicine, Geisinger Health System, Danville, PA

Background/Aims: New evidence supports complex care management teams to improve outcomes and reduce costs for the small proportion of patients who account for a majority of health care expenditures. However, there is a lack of evidence specific to the adolescents and young adults with special health care needs in the transition age group, most of whom are covered by Medicaid. Geisinger has implemented a new care model - the Comprehensive Care Clinic (CCC) - focused on such patients. The CCC is comprised of a care team consisting of a super-generalist physician, advanced practitioner, pharmacist and a nurse case manager who provides close follow-up. Its clinical redesign includes advanced visit types, clinic hours for emergent situations and provider time outside of visits to coordinate interactions of the CCC team members with the patient, family caregiver and other health care providers. A central feature of the $\mathrm{CCC}$ is its reliance on a single care plan tailored to each patient to plan collaboratively for preventive and routine care as well as managing exacerbation and facilitating self-management education.

Methods: Geisinger Health Plan claims data covering a 19-month period ending September 31, 2014, were obtained for the 83 Medicaid patients enrolled in CCC as of April 2015, yielding 1,425 member-month observations. The dependent variables were total expenditure (allowed amounts including prescription drugs) and acute care utilization (inpatient admissions and emergency room [ER] visits). The key explanatory variable was an indicator for whether the member was enrolled in CCC in a given month of the study period. Multivariate regression models with patient fixed effects were estimated to obtain adjusted differences in cost and utilization between months in which the patients were enrolled and not enrolled in the CCC.

Results: CCC enrollment was associated with $28 \%$ reduction 
in per-member-per-month total cost $(\$ 3,931$ observed vs. $\$ 5,451$ expected, $\mathrm{P}=0.028$ ), driven by large reductions in acute inpatient admissions and ER visits.

Conclusion: A clinical redesign involving longer visits, clinic time for emergent needs, a coordinated care plan and a partnership among super generalists, nurse care manager and pharmacists can potentially reduce total cost and acute care utilization during transition from pediatrics to adult medicine. Keywords: medically complex, Medicaid

\section{B4-2:}

\section{Hepatitis C Virus Infection and Increasing Screening and Linkage to Care in a Large Integrated Health System}

Carla V. Rodriguez, ${ }^{1}$ Kevin B. Rubenstein, ${ }^{1}$ Haihong $\mathrm{Hu},{ }^{1}$ Benjamin P. Linas, ${ }^{2}$ Michael Horberg ${ }^{1}$

\section{${ }^{1}$ Mid-Atlantic Permanente Research Institute, Kaiser Permanente Mid-Atlantic States, Rockville, MD; ${ }^{2}$ Department of Infectious Diseases, Boston University School of Medicine, Boston, MA}

Background/Aims: With increased national focus on diagnosing and linking patients to care or treatment, we describe trends in hepatitis $\mathrm{C}$ virus (HCV) screening and linkage to care in the Kaiser Permanente Mid-Atlantic States health system, particularly among those born between 1945 and 1965 (birth cohort). We observed 665,345 adults enrolled in Kaiser Permanente Mid-Atlantic States from 2003 to 2015. Methods: We used survival methods to describe factors associated with time to antibody and confirmatory (RNA/ genotype) testing. Logistic regression was used to describe odds of $\mathrm{HCV}$ infection and gastroenterology/infectious disease visit or treatment (linkage).

Results: The annual screening rate increased steadily from 23.6 to 70.8 per 1,000 person-years, with the sharpest increase after 2013. Overall, 19\% (123,572 of 665,345) were screened for HCV. Screening among the birth cohort was lower than among noncohort members. However, the gap shrank in later years of study. Significant positive predictors of screening included drug use, hepatitis B and human immunodeficiency virus (HIV) status, female sex, living in lower-income areas and elevated alanine aminotransferase (ALT). Among antibodypositive adults, $86 \%(3,643$ of 4,242) received confirmatory testing. A prior gastroenterology/infectious disease visit and being seen in Baltimore clinics were associated with increased rates of confirmatory testing. We identified 3,310 with chronic HCV by RNA or genotype $(2.7 \%$ of the 124,841 persons ever antibody-tested, RNA-tested or genotyped). Blacks, birth cohort members, males, those with history of illicit drug use and those with elevated ALT were associated with higher odds of HCV infection. Hispanics, patients with HIV or hepatitis B, and those from higher-income areas were at lower risk. Among patients with chronic HCV, 86\% $(2,844$ of 3,310 ) were linked to care. Predictors of linkage include elevated ALT, HIV status and black or Hispanic race.

Conclusion: We report higher rates of $\mathrm{HCV}$ screening, confirmatory testing and linkage compared to similar cohorts. Although HCV screening is still predominantly risk-based, birth cohort screening is increasing in concert with national guidelines. Our data support other studies that suggest that sexual intercourse, even among men who have sex with men, is not a primary means of HCV transmission, and that risk of HCV is higher in low-income areas. These data provide important parameters from which to base and compare future interventions to increase screening, care and treatment.

Keywords: hepatitis C, epidemiology

\section{B4-3:}

Working Towards De-Implementation: A MixedMethods Study in Breast Cancer Surveillance Care

Erin E. Hahn, ${ }^{1}$ Corrine E. Munoz-Plaza, ${ }^{1}$ Jianjian Wang, ${ }^{1}$ Jazmine Garcia Delgadillo, ${ }^{1}$ Brian S. Mittman, ${ }^{1}$ Michael K. Gould ${ }^{1}$

\section{${ }^{1}$ Department of Research \& Evaluation, Kaiser Permanente Southern California, Pasadena, CA}

Background/Aims: De-implementing commonly used but ineffective clinical practices is an important component of quality. Oncology offers several opportunities to reduce use of ineffective practices based on guidelines from the American Society of Clinical Oncology (ASCO). We studied the use of one such practice, biomarker blood tests for breast cancer surveillance, within an integrated health care system. We documented utilization patterns and explored provider perceptions and attitudes to inform de-implementation efforts. Methods: Using a sequential explanatory mixed-methods design, we identified a cohort of early-stage breast cancer survivors and calculated the number and frequency of biomarker tests during an 18-month posttreatment period. We identified high- and low-utilizing medical centers and conducted semistructured qualitative interviews with oncologists in both types of centers, guided by the Theoretical Domains Framework. Interviews were transcribed, coded and analyzed.

Results: Among 7,363 patients diagnosed during 2009-2012, 40,114 biomarker tests were ordered for $41 \%$ of patients. We found significant variation by medical center: $5-78 \%$ of eligible patients received a test. We interviewed 18 oncologists in highand low-utilizing centers. Several themes emerged, including: 1) Awareness of nonadherence: oncologists reported awareness of current ASCO guidelines and agreed that biomarkers are not clinically useful; high utilizers acknowledge intentional nonadherence ("We all know we shouldn't do this but do it anyways."); 2) Anxiety: despite agreeing that biomarkers aren't useful, some oncologists are anxious about missing a recurrence and want to do "all possible" to prevent this; high utilizers perceive that patients are highly anxious and desire a quantitative test for reassurance ("They need a number."); and 3) Perceived patient expectations: oncologists perceive competition from other systems and are concerned about perception of withholding care ("If [competitor] does it ... patients expect it.") and implications for patient satisfaction. Conclusion: Barriers to de-implementation are numerous and 
complex. Traditional strategies of practice change based on increasing awareness and knowledge (provider education, electronic alerts) are unlikely to be effective. Multifaceted, multilevel strategies deployed to address consumer-, clinicianand system-related barriers are required. Research-based development and evaluation of multilevel de-implementation strategies is critical and will help develop valuable insights and theories regarding the determinants of clinical practices and opportunities to influence them.

Keywords: implementation science, mixed-methods research

\section{B4-4:}

\section{Care Redesign in Joint Care}

Tamara Cull, ${ }^{1}$ Steve Brown, ${ }^{1}$ Samantha Mochaitis, ${ }^{1}$ Greg Rennirt ${ }^{2}$

\section{${ }^{1}$ Catholic Health Initiatives, Englewood, CO; ${ }^{2}$ KentuckyOne Health, Louisville, KY}

Background/Aims: The goals of population health are to improve the health status of defined populations by focusing on quality, cost and experience of care (Triple Aim). With the Centers for Medicare \& Medicaid Services' development of bundled payment programs (e.g. Bundled Payments for Care Improvement, Comprehensive Care for Joint Replacement) as well as other quality-focused advances in the industry, there are increased avenues to incentivize physician and hospitals to collaborate to achieve common goals around quality, experience and cost.

Methods: The efforts focused on leveraging a 9-step care redesign model to evaluate best practices in joint care across 11 state health care systems. Workflows were evaluated and opportunities for care improvement were identified and implemented. The Total Knee Best Practice Change Assessment brought about enhancements, which included the engagement of care management/navigation beginning at the preoperative stage and continuing through the entire episode of care. These efforts included robust patient optimization, education and engagement while leveraging effective analytics to inform our efforts.

Results: Programs that significantly lowered post-acute care costs (specifically, costs related to admission to skilled nursing facilities) achieved substantial savings in post-acute care. In addition, we observed a decrease in readmissions, a decrease in skilled nursing facility utilization and an increase in patient satisfaction. Key lessons learned include: a) the significance of physician leadership in implementing organizational change, and b) the importance of $24 / 7$ patient access to care management to reduce emergency department utilization.

Conclusion: Findings from this study have direct implications for ongoing episode-based payment initiatives aimed at improving quality and decreasing costs, as they suggest that episode-based payment models have the potential to decrease total costs per episode.

Keywords: care redesign, value
PS1-23:

A Depression Care Management Project to Improve Treatment Coordination and Outcomes in Patients With Comorbid Conditions

John E. Zeber, ${ }^{1}$ Laurel A. Copeland, ${ }^{1}$ Duke Ruktanonchai, ${ }^{2}$ I-Chia Liao, ${ }^{1}$ Judy Embry ${ }^{3}$

${ }^{1}$ Center for Applied Health Research, Baylor Scott \& White Health, Temple, TX; ${ }^{2}$ Baylor Scott \& White Health Plan, Temple, TX; ${ }^{3}$ Department of Family Medicine, Baylor Scott \& White Health, Temple, TX

Background/Aims: Appropriate clinical management of major depression and comorbid medical conditions represents a significant challenge for providers. Adopting a patent-centered treatment perspective, qualified nonphysician behavioral health specialists can support monitoring complex needs, enhance care provision and satisfaction with medical encounters, and improve costeffective outcomes. This demonstration project evaluated a depression management program, examining effects on depressive symptoms, chronic disease and medication management, utilization and treatment costs.

Methods: Beginning January 2015, the program offers enrollment to primary care patients scoring above 15 on the Patient Health Questionnaire-9 (PHQ-9). Individuals receive an integrated, holistic health care plan, psychiatric consultations as needed, education concerning medications and side effects, and proactive follow-up. Besides facilitating team communication, the care manager provides evidence-based brief counseling (e.g. motivational interviewing, behavioral activation) and initiates treatment modifications to optimize clinical gains. As participants complete 6 months or achieve remission of depression, we evaluate changes in PHQ-9 and satisfaction ratings while linking to administrative data on utilization, prescriptions and costs.

Results: To date, 58 patients are enrolled, with mean age of 51.5 (range: 12-88) and $72 \%$ women. Among early notable findings, substantial improvements were observed in abatement of clinical depression. Of 48 individuals with subsequent PHQ-9s, their mean decline was 7.7 points; $50 \%$ exhibited remittance and $86 \%$ showed clinical improvement. Other analysis suggests a slight drop in emergency visits and fewer prescriptions across most psychotropic and medical drug classes, controlling for program duration. Patient satisfaction with the program is quite positive, especially perceived inclusion when discussing treatment strategies and provider attention to patient mood. Ongoing work will examine changes in specialty mental health care, admissions and costs in comparison to a similar clinic cohort, matching for demographics and clinical characteristics.

Conclusion: Consistent with national efforts to better engage patients in their health care decisions, this care management program employs highly qualified, lower-cost providers for most encounters. Such personalized, coordinated treatment of comorbid conditions within a primary care environment offers tremendous promise for improving depression and 
quality of life while proactively reducing unnecessary treatment and system costs. Further longitudinal analyses will monitor these impressive short-term benefits as we fully examine implementation of a truly patient-centered, feasible care approach.

Keywords: depression, care management

\section{PS1-24:}

Timeliness of Follow-Up to a Positive Fecal Immunochemical Test Result Among Community Health Center Patients

\section{Ann Oluloro, ${ }^{1}$ Amanda F. Petrik, ${ }^{1}$ Gloria Coronado, ${ }^{1}$ Tanya Kapka, ${ }^{2}$ Jennifer S. Rivelli ${ }^{1}$ \\ ${ }^{1}$ Center for Health Research, Kaiser Permanente Northwest, Portland, OR; ${ }^{2}$ Virginia Garcia Memorial Health, Cornelius, OR}

Background/Aims: Colorectal cancer is the fourth most common cancer and the third-leading cause of cancer death in the United States. Fecal testing, including fecal immunochemical testing (FIT), has been proven to reduce mortality from colorectal cancer. Such mortality reductions can only be achieved, however, if those with abnormal test results receive follow-up colonoscopies. Completion rates for follow-up colonoscopy are low, especially in community health centers, where many Hispanics receive care. As part of the Strategies and Opportunities to STOP Colon Cancer in Priority Populations (STOP CRC) project, we examined rates of adherence to follow-up colonoscopy, time to colonoscopy completion and characteristics of patients who complete colonoscopy after abnormal FIT results compared to those who do not.

Methods: Virginia Garcia Memorial Health Center was the clinic partner in this project. Project staff reviewed charts from patients who had abnormal FIT results following the STOP CRC outreach program. Reviews of electronic medical charts ascertained patient demographic characteristics, referral to colonoscopy (yes/no), date of referral and reason (if not made), colonoscopy completion (yes/no) and date of colonoscopy and reason (if not completed). Bivariate analyses and regression analyses were used to examine associations and complete mediator analysis.

Results: A total of 56 patients had abnormal FIT results; $29(52 \%)$ were Hispanic and 31 (55\%) were female. Fortyfive $(80 \%)$ patients received referral for colonoscopy, with a median time to referral of 2 days. Of the 56 patients, 32 (57\%) had evidence of a completed colonoscopy in their medical charts. Latinos were less likely than non-Latino whites to have completed a colonoscopy (44.8\% vs. $70.4 \%)$. The median time to colonoscopy completion was 62 days. Females were less likely than males to complete their colonoscopy within 60 days of a positive FIT result (odds ratio: $0.21,95 \%$ confidence interval: $0.05-0.96$ ). Mediation analysis indicated that time to referral was not a mediator between patient-level factors and completion of follow-up colonoscopy.
Conclusion: Our findings suggest improvements are needed to increase rates of follow-up colonoscopy completion, especially among females and Hispanic patients. Future research might explore the role that clinic- and patient-level factors play in colonoscopy completion.

Keywords: federally qualified health center, colorectal cancer

\section{PS1-26: \\ Understanding Asian Patients' Lower Satisfaction With Health Care}

Lu Wah Hung, ${ }^{1}$ Katherine Gillespie, ${ }^{1}$ Shuting Liang, ${ }^{1}$ Rachel Schwartz, ${ }^{1}$ Sukyung Chung, ${ }^{1}$ Meghan C. Halley ${ }^{1}$

${ }^{1}$ Palo Alto Medical Foundation Research Institute, Palo Alto Medical Foundation for Health Care, Research and Education, Palo Alto, CA

Background/Aims: Asians now exceed Latinos as the highest proportion of new immigrants in the United States, totaling over 18 million. Asian patients report lower satisfaction with their health care than do non-Hispanic white (NHW) patients. The goal of this comparative, mixed-methods study was to explore Asian patients' expectations and experiences with health care compared to NHW patients as well as how cultural norms related to evaluation may contribute to lower patient satisfaction among Asians.

Methods: From July 2014 to August 2015 we conducted a comparative mixed-methods study with Chinese, South Asian and NHW patients from a large multispecialty clinic in Northern California. Electronic health records were used to select patients for recruitment, and invitations were sent via letter, phone and/or email. Chinese patients include those whose preferred language was Mandarin, Cantonese or English. As English is widely spoken among the South Asian patient population, these patients were screened for acculturation at recruitment to include equal numbers of high- and low-acculturation patients. Patients participated in either a focus group or cognitive interview, and all participants completed surveys including demographics and standardized measures of acculturation, health care expectations and satisfaction. Focus group participants discussed their expectations and experiences of care, while cognitive interview participants shared the cognitive processes underlying their evaluation of a standardized health care visit vignette. Focus groups and surveys for Chinese patients were conducted in their preferred language. All cognitive interviews were conducted in English.

Results: We conducted focus groups with 58 Chinese (21 English-, 21 Mandarin-, and 16 Cantonese-speaking), 27 South Asian (14 high and 13 low acculturation) and 12 NHW patients, and cognitive interviews with 11 Chinese, 13 South Asian and $10 \mathrm{NHW}$ patients (total $\mathrm{N}=131$ ). A comparative mixed-methods analysis is currently underway, employing descriptive and bivariate statistics and an integrated deductive and inductive thematic analysis. Results will compare subgroups of Asian and NHW patients in terms 
of: 1) expectations and experiences with health care, and 2) cognitive processes underlying evaluation of health care experiences.

Conclusion: Pending results will provide a comparative examination of differences between Asian and NHW patients' reported satisfaction with care and the factors underlying these differences.

Keywords: patient satisfaction, racial/ethnic differences

\section{PS1-27:}

Patterns of Multiple Emergency Department Visits: A Discretionary Care-Seeking Behavior?

\author{
Daniel D. Maeng, ${ }^{1}$ John B. Bulger ${ }^{2}$ \\ ${ }^{1}$ Geisinger Center for Health Research, Danville, PA; \\ ${ }^{2}$ Geisinger Health System, Geisinger Health Plan, \\ Danville, PA
}

Background/Aims: Identifying an effective strategy to reduce emergency department (ED) visits remains challenging. This study seeks to empirically identify patient characteristics and utilization patterns that are significantly associated with multiple ED visits by patients within a 1 -year period. The rationale is that a single ER visit is likely to be subject to random variation that is inherently difficult to predict. This study focuses only on Geisinger Health Plan members who had at least one ED visit in a 2-year period (2013-2014) across all plan types for which Geisinger Health Plan was "at risk" (i.e. Medicaid, Medicare, commercial, the Exchange) and identifies patient characteristics and other care utilizations (e.g. high-end diagnostic imaging, outpatient surgery, physician office visits) that are associated with multiple ED visits within a 12-month period.

Methods: The sample was restricted to adult Geisinger Health Plan members ( $\geq 18$ years old) and included 67,997 unique members. Two multivariate logistic regression models were estimated: 1) a model that includes primary care physician (PCP) fixed effects, and 2) another model that does not include PCP fixed effects. The PCP fixed effects were used to examine if the PCP influences the likelihood of multiple ED visits.

Results: More frequent physician office visits were associated with greater likelihood of multiple ED visits $(\mathrm{P}<0.001)$. Multiple use of high-end diagnostic imaging (computed tomography and/or magnetic resonance imaging) and multiple outpatient surgeries also were both associated with greater likelihood of multiple ED visits $(\mathrm{P}<0.001)$. These associations remain significant even after controlling for age (older patients were less likely to make multiple ED visits), gender, plan types (Medicaid members were more likely to have multiple ED visits than members with other plan types) and chronic conditions. The odds ratios obtained with and without PCP fixed effects were similar, indicating that PCPs do not contribute significantly to patients' likelihoods of multiple ED visits.

Conclusion: These patterns suggest that some patients may be seeking outpatient care that is not explained by their clinical and demographical characteristics alone. More studies are necessary to explore the underlying mechanism that explains such an apparent care-seeking behavior.

Keywords: emergency department, claims data

PS1-28:

Validation of Colorectal Cancer Screening in the Electronic Health Record for Identifying Patients Due for Screening in a Pragmatic Trial

Amanda F. Petrik, ${ }^{1}$ Bev Green, ${ }^{2}$ William Vollmer, ${ }^{1}$ Thuy Let, ${ }^{3}$ Barbara Bachman, ${ }^{1}$ Erin Keast, ${ }^{1}$ Gloria Coronado ${ }^{1}$

${ }^{1}$ Center for Health Research, Kaiser Permanente Northwest, Portland, OR; ${ }^{2}$ Group Health Research Institute, Group Health Cooperative, Seattle, WA; ${ }^{3} \mathrm{OCHIN}$, Portland, OR

Background/Aims: In 2015 an estimated 143,000 adults in the United States will be diagnosed with colorectal cancer, and 52,000 will die from the disease. Despite this knowledge, colorectal cancer screening rates remain low. Electronic health records (EHR) hold much promise for helping to close this gap by identifying eligible individuals who are overdue for or have never completed colorectal cancer screening; however, important shortfalls to this approach remain. Records of colonoscopy completion are frequently missing in the EHR. Variation in workflow and documentation can lead to incomplete capture of colorectal cancer screening and testing events. Through the Strategies and Opportunities to STOP Colon Cancer in Priority Populations (STOP CRC) study, we conducted a data validation to understand the data being captured in the EHR and completeness of the data.

Methods: We selected a stratified random sample of 800 study participants from 26 participating clinics to compare the EHR to chart audit data. A trained validation specialist completed the abstraction qualifying data of eligible and ineligible patients.

Results: Comparing EHR data to chart audits, we found 88\% $(459 / 520)$ of individuals were correctly classified as eligible for program inclusion. EHR data correctly identified $96 \%$ (269/280) of excluded patients. Of the patients incorrectly classified as eligible, $83.6 \%(51 / 61)$ of disagreements were due to evidence of a prior colonoscopy or referral that was not captured in recognizable fields in the EHR.

Conclusion: If our goal to increase CRC screening uptake is achieved, there will be an even greater need to improve data capture of all screening events, document completion of diagnostic testing after a positive test, conduct surveillance exams and provide appropriate outreach to patients needing repeated screening. While the need for better populationbased data is not unique to CRC screening, it provides an important example of using population-based data for not only tracking needed care but also directly delivering needed interventions.

Keywords: validation, electronic health record 


\section{CHRONIC CONDITIONS, OBESITY, DIABETES, CARDIOVASCULAR DISEASE}

A1-1:

\section{Relation of Change in Weight Status to the Development of Hypertension in Children and Adolescents}

Patrick J O'Connor, ${ }^{1}$ Emily D. Parker, ${ }^{1}$ Alan R. Sinaiko, ${ }^{2}$ Elyse O. Kharbanda, ${ }^{1}$ Karen L. Margolis, ${ }^{1}$ Matthew F. Daley, ${ }^{1}$ Nicole K. Trower, ${ }^{1}$ Nancy E. Sherwood, ${ }^{1}$ Louise C. Greenspan, ${ }^{3,4}$ Joan C. Lo, ${ }^{3}$ David J. Magid ${ }^{5}$

${ }^{1}$ HealthPartners Institute for Education and Research, Minneapolis, MN; ${ }^{2}$ Department of Pediatrics, University of Minnesota, Minneapolis, MN; ${ }^{3}$ Kaiser Permanente Northern California, Oakland, CA; ${ }^{4}$ University of California, San Francisco, San Francisco, CA; ${ }^{5}$ Institute for Health Research, Kaiser Permanente Colorado, Denver, CO

Background/Aims: This study examined the association of body mass index (BMI) percentile and change in BMI percentile to change in blood pressure (BP) percentile and development of hypertension in children and adolescents.

Methods: This retrospective cohort included 101,725 subjects aged 3-17 years from three health systems across the United States. Height, weight, age, sex and BP measures were extracted from electronic health records, and then age/ sex/height-adjusted BP percentiles and BMI percentiles were computed. Mixed linear regression estimated change in systolic BP percentile, and proportional hazards regression was used to estimate risk of incident hypertension associated with BMI percentile and change in BMI percentile.

Results: The largest increases in BP percentile were observed among children and adolescents who became obese or maintained obesity. Over a median 3.1-year follow-up, $0.4 \%$ of subjects developed hypertension. Obese children aged 3-11 had 3.5-fold increased risk of developing hypertension compared with normal weight. Obese adolescents aged 12-17 had 3.2fold increased risk of developing hypertension compared with normal weight. Children and adolescents who stayed obese had 5.4- and 4.8-fold increased risk of developing hypertension, respectively, compared with those who maintained a normal weight. Children who became obese and adolescents who became overweight had 2.6- and 2.3-fold increased risk of developing hypertension, respectively.

Conclusion: We observed a strong, statistically significant association between increasing BMI percentile and increases in BP percentile, with risk of incident hypertension primarily associated with obesity. The adverse impact of weight gain and obesity in this young cohort over a short period of time underscores the need for effective strategies for prevention of overweight and obesity in youth to slow progression toward diabetes and cardiovascular disease later in life.

Keywords: pediatrics, obesity
A1-2:

Five-Year Weight Change Trajectories in Roux-en-Y Gastric Bypass Patients

Michelle R. Lent, ${ }^{1}$ G. Craig Wood, ${ }^{1}$ Adam Cook, ${ }^{1} \mathrm{H}$. Lester Kirchner, ${ }^{1}$ Sharon Larson, ${ }^{1}$ David B. Sarwer, ${ }^{2}$ Christopher D. Still ${ }^{1}$

${ }^{1}$ Center for Health Research, Geisinger Health System, Danville, PA; ${ }^{2}$ Center for Obesity Research and Education, Temple University, Philadelphia, PA

Background/Aims: Roux-en-Y gastric bypass (RYGB) is a popular weight-loss procedure, but not all patients experience optimal long-term outcomes. Previous studies indicate that bariatric surgery patients with similar preoperative profiles may respond differently to the same surgical intervention for severe obesity. This study's primary aim was to identify long-term weight change trajectories in a large cohort of RYGB patients over 5 years.

Methods: This longitudinal study followed adult RYGB patients preoperatively to 5 years postoperatively. Patients were recruited from 2004 to 2014 from the bariatric surgery program of a large comprehensive rural medical center. Demographic and weight information was obtained through the electronic medical record. The study's primary outcome, percent total body weight loss (\% TBWL), was calculated using the weight at time of surgery and all weights available in the electronic medical record as part of routine clinical care through 5 years after surgery. Weight loss trajectories were then estimated using a semiparametric latent class growth modeling approach.

Results: The majority of RYGB participants $(\mathrm{N}=3,215)$ were female $(80 \%, 2,575$ of 3,215$)$; mean preoperative body mass index was $49.4 \pm 8.8 \mathrm{~kg} / \mathrm{m}^{2}$. Four distinct weight loss trajectories were identified for the 5 years following RYGB. In our sample, $16.8 \%(541$ of 3,215$)$ of participants failed to maintain $20 \%$ of their initial weight loss, representing a "suboptimal" weight loss trajectory group. Two additional groups (combined $15.9 \%, 513$ of 3,215) reached similar weight loss endpoints at 5 years (approximately 40\% TBWL), with a subset of these participants following a slower but steady trajectory $(7.6 \%, 245$ of 3,215$)$. Finally, a fourth group $(67.2 \%, 2,161$ of 3,215$)$ reached a weight loss nadir of about $30 \%$ and maintained $25 \%$ weight loss at Year 5.

Conclusion: Weight change trajectories after RYGB are not uniform, with some patients experiencing suboptimal weight loss of less than $20 \%$ by Year 5 . Greater understanding of the individual-level characteristics that distinguish patients with suboptimal weight change may help to improve patient and procedure selection.

Keywords: obesity, bariatric surgery 
A1-3:

Improving Confidence to Eat Better Among Young Adults - Gender Differences in 3-Month Results of the MENU GenY Online Intervention

\section{Gwen Alexander, ${ }^{1}$ Andrew Taylor, ${ }^{1}$ Margaret Rukstalis, ${ }^{2}$ Ken Resnicow ${ }^{3}$ \\ ${ }^{1}$ Department of Public Health Sciences, Henry Ford Health System, Detroit, MI; ${ }^{2}$ Center for Health Research, Geisinger Health System, Danville, PA; ${ }^{3}$ Center for Health Communications Research, University of Michigan, Ann Arbor, MI}

Background/Aims: Improving food choices requires both internal resources, particularly confidence in ability to change, and external challenges or temptations. Selfefficacy and social influence, especially through informal close relationships, play important roles in health behavior. We assessed dietary and self-efficacy changes related to dietary choices by gender and initial fruit and vegetable intake among young adults enrolled in a randomized online intervention trial to improve food choices.

Methods: Young adults, 21-30 years old, from integrated health systems in urban Michigan and rural Pennsylvania enrolled and were randomized into Arm 1 (control website), Arm 2 (tailored and age-targeted website) or Arm 3 (Arm 2 website + email coaching). Baseline and 3-month online surveys included questions assessing fruit and vegetable $(\mathrm{F} / \mathrm{V})$ intake, and confidence about eating healthy foods in a number of circumstances (e.g. when really hungry, tired, with friends). Repeated measures ANOVA and F-tests compared overall and groups by study arm, gender and baseline $\mathrm{F} / \mathrm{V}$ intake.

Results: Of 1,390 ( $81 \%$ of baseline, $71 \%$ women) completing the 3-month survey, mean gain in $\mathrm{F} / \mathrm{V}$ intake was 1.0 (SD: 1.4), 1.0 (SD: 1.5) and 1.2 (SD: 1.5) servings by Arms 1, 2 and 3, respectively; those with low (1 or fewer) $\mathrm{F} / \mathrm{V}$ at baseline $(\mathrm{n}=153)$ improved by $2+$ servings, across arms, with no differences by gender. Greatest self-efficacy improvement, regardless of gender, was in eating well in front of friends $(\mathrm{P}<0.0001)$, making good choices eating out $(\mathrm{P}<0.0001)$ and eating healthy when depressed/in a bad mood $(\mathrm{P}<0.0001)$; men also gained in self-efficacy to eat healthy around junk food $(\mathrm{P}<0.001)$. The low baseline $\mathrm{F} / \mathrm{V}$ group resembled men and gained self-efficacy to prepare healthy meals $(\mathrm{P}<0.001)$ and avoid higher-fat foods.

Conclusion: Internal perceptions of self-efficacy across a number of social situations and internal conditions in both men and women corresponded to personal experience of working to eat more F/V. More self-confidence improvements occurred in those starting with lowest rates of healthy eating, and those who ultimately made the greatest gains in dietary change, regardless of gender.

Keywords: nutrition, young adults
A1-4:

Qualitative Data from a Trial of Home Blood Pressure Telemonitoring and Pharmacist Management (Hyperlink)

Anna R. Bergdall, ${ }^{1}$ JoAnn M. Sperl-Hillen, ${ }^{1}$ Patrick J. O'Connor, ${ }^{1}$ Steve E. Asche, ${ }^{1}$ Benjamin F. Crabtree, ${ }^{2}$ Emily A. Smith, ${ }^{3}$ Rachel A. Nyober, ${ }^{1}$ Steven P. Dehmer, ${ }^{1}$ Michael V. Maciosek, ${ }^{1}$ Pamela A. Pawlowski, ${ }^{1}$ Nicole K. Trower, ${ }^{1}$ Karen L. Margolis ${ }^{1}$

${ }^{1}$ HealthPartners Institute for Education and Research, Minneapolis, MN; ${ }^{2}$ Department of Research \& Education, Rutgers Robert Wood Johnson Medical School, New Brunswick, NJ; ${ }^{3}$ University of Minnesota School of Public Health, Minneapolis, MN

Background/Aims: Hyperlink was a cluster-randomized intervention trial in HealthPartners clinics from 2009 to 2013 with nonintervention follow-up through 2015 (60 months). Participants had uncontrolled hypertension. Telemonitoring intervention patients had improved blood pressure control at 6 months compared with usual care patients $(72 \%$ vs. $45 \%, \mathrm{P}<0.001)$. Intervention effects narrowed at $12(72 \%$ vs. $53 \%, \mathrm{P}=0.005)$ and 18 months ( $72 \%$ vs. $57 \%, \mathrm{P}=0.003)$; 60-month blood pressure data will be complete in October 2015. We conducted a mixed-methods analysis combining our quantitative results with patient, clinical and other organizational stakeholder perspectives to learn how to optimize the intervention for the most patients and implement this intervention in our care setting.

Methods: We collected three sources of qualitative data: seven patient focus groups stratified by 6-18-month blood pressure outcomes, four structured interviews with intervention pharmacists, and interviews (currently being collected) with key organizational stakeholders. Focus group and structured interview data were analyzed by a team of five using grounded theory. Initial themes were identified and coded in NVivo10.

Results: Qualitative data revealed several initial themes. First, patients valued trust in the patient-provider relationship and good communication between providers. Second, patients have varying goals with medications and successfully initiating/adhering to treatment is better when provider understands and respects the patient's perspective on medications. Finally, intervention patients benefited from seeing their own blood pressure data (reinforcement) and a trusted provider seeing their data (accountability). Pharmacist interviews agreed with these themes, revealing key insights about intervention design including: length of intervention, addressing relapse, and meeting individual patient's needs with effective use of data and lifestyle counseling. Results of 60-month blood pressure outcomes will be analyzed in the context of these initial findings, and qualitative findings will be further refined. Stakeholder interview results about implementation are forthcoming.

Conclusion: Findings suggest the need for several adaptations to the intervention before implementation in practice: provision of blood pressure monitors for ongoing use, a shorter duration with ability to re-engage if blood pressure 
becomes uncontrolled, more tailoring of the intervention to individual needs, and better communication and handoffs between pharmacists and physicians.

Keywords: telemonitoring, hyperlink

B1-1:

Preventive Service Use Among People With Serious Mental IIInesses: Results From the PRIME Study

Bobbi Jo. Yarborough, ${ }^{1}$ Nancy Perrin, ${ }^{1}$ Elizabeth Shuster, ${ }^{1}$ Scott P. Stumbo, ${ }^{1}$ Micah T. Yarborough, ${ }^{1}$ Carla A. Green ${ }^{1}$

${ }^{1}$ Center for Health Research, Kaiser Permanente Northwest, Portland, OR

Background/Aims: People with serious mental illnesses (SMI) experience excess morbidity and premature mortality resulting from preventable conditions. In response, concerns have been raised that people with SMI may not receive adequate preventive services. Understanding preventive service use patterns may help improve care delivery and reduce health disparities in this population.

Methods: Participants included all adults $\geq 18$ years with at least one prior 12-month health care visit in a large integrated service system (Kaiser Permanente Northwest [KPNW], $\mathrm{n}=366,194)$ or in participating federally qualified health centers and safety-net clinics in 18 states (OCHIN, n=437,082). We used electronic medical record data to examine receipt of needed preventive care, measured by 12 services (e.g. flu shots, mammogram screening). We computed proportion of needed care by dividing the number of out-of-date services by the number of eligible services and multiplying by 100. Mean rates for four diagnostic groups - schizophrenia spectrum disorders, bipolar disorders/affective psychoses, major depressive disorders, anxiety disorders - were compared to patients without SMI diagnoses. All models were adjusted for patient characteristics (e.g. age, gender, Medicaid/Medicare status) and service use.

Results: At OCHIN, all SMI groups had lower rates of needed preventive services (schizophrenia: 34.12, bipolar disorders/ affective psychoses: 35.68, major depressive disorders: 36.30, anxiety: 38.53) than non-SMI patients (39.97). Similarly, needed services did not differ or were lower among KPNW members with SMI, with bipolar disorders/affective psychoses (18.64) and major depressive disorders (18.63) showing significantly lower needed services than non-SMI members (20.64). Proportions of individuals with SMI completing cholesterol and diabetes screening were higher than those without SMI at both sites. At KPNW, individuals with SMI were more likely to have a recorded body mass index than individuals without SMI, but at OCHIN the reverse pattern was observed. Colorectal cancer screening did not differ by diagnosis but was more likely to occur at KPNW than at OCHIN. Blood pressure screening was common across diagnostic groups and sites. Flu vaccinations were uncommon, particularly at OCHIN.

Conclusion: Contrary to hypotheses, individuals with serious mental illnesses received more preventive services than individuals without mental illnesses. Adjusting for number of health care visits reduced, but did not eliminate, these differences.

Keywords: preventive service use, serious mental illness

B1-2:

Abdominal Aortic Calcification on Lateral Spine Bone Density Test Images: Potential Role in Cardiovascular Disease Risk Screening

John T. Schousboe, ${ }^{1}$ Mary Sue Beran ${ }^{1}$

${ }^{1}$ Park Nicollet Institute, Minneapolis, MN

Background/Aims: Abdominal aortic calcification (AAC) is associated with incident clinical cardiovascular disease independent of other clinical risk factors. The aorta lies immediately anterior to the lumbar spine, and AAC can be accurately scored on bone density lateral spine images. Our objective was to estimate the proportion of individuals $65-80$ years old undergoing bone densitometry who are not known a priori to be at high risk of cardiovascular disease (based on prior diagnoses and Framingham hard coronary heart disease [CHD] 10-year risk score), but who have a high level of AAC (AAC-24 Framingham score $\geq 5$ ).

Methods: AAC was scored on lateral spine bone density images for 1,499 randomly selected patients age $65-80$ at a large urban community health care delivery organization, blinded to patient characteristics. Established diagnoses of cardiovascular disease or diabetes mellitus were determined by identification of appropriate ICD-9 diagnosis codes at provider visits. Framingham 10-year hard CHD risk scores were calculated from clinical data (systolic blood pressure, total and high-density-lipoprotein cholesterol, smoking status, use of antihypertensive medication) extracted from the electronic health record.

Results: Mean age of the study cohort was 71 years; $92.9 \%$ were female, $94.1 \%$ were Caucasian, $14.0 \%$ had preexisting cardiovascular disease, $10.7 \%$ had preexisting diabetes mellitus, and $24.7 \%$ had a Framingham hard CHD risk score $\geq 7.5 \%$. A total of 490 patients $(32.7 \%)$ had no AAC, 603 $(40.2 \%)$ had mild to moderate AAC (AAC-24 score of 1 to 4), and 406 (27.1\%) had a high level of AAC; 184 patients $(12.3 \%)$ had both a high level of AAC and were not previously known to be at high risk based on preexisting clinical cardiovascular disease, diabetes mellitus or a Framingham hard CHD risk score $\geq 7.5 \%$.

Conclusion: The proportion of those age 65 to 80 undergoing bone densitometry who are not known to be at high risk of incident cardiovascular disease but who have high AAC is sufficient that densitometric lateral spine imaging at the time of bone densitometry may have a role in cardiovascular disease risk screening, considering bone densitometry is recommended at least once for all women $\geq 65$ years old and men $\geq 70$ years.

Keywords: abdominal aortic calcification, incident clinical cardiovascular disease 
B1-3:

Overuse and Underuse of Aspirin for Primary Prevention of Cardiovascular Events in Primary Care

JoAnn M. Sperl-Hillen, ${ }^{1}$ Patrick J. O'Connor, ${ }^{1}$ Karen L. Margolis, ${ }^{1}$ Alan L. Crain, ${ }^{1}$ Heidi L. Ekstrom ${ }^{1}$

\section{${ }^{1}$ HealthPartners Institute for Education and Research, Minneapolis, $M N$}

Background/Aims: The U.S. Preventive Services Task Force (USPSTF) currently recommends aspirin for primary prevention of coronary heart disease in men 45-79 years old and strokes in women 55-79 years old when the potential cardiovascular disease benefit outweighs the potential harm of gastrointestinal hemorrhage. The complexity and time required to assess risks and benefits for primary prevention can be a barrier for providers to giving patients USPSTF-consistent recommendations, resulting in potential overuse and underuse.

Methods: As part of a National Institutes of Health-funded randomized trial to lower cardiovascular risk, we developed a sophisticated web-based electronic health record (EHR)integrated tool to guide aspirin recommendations as determined by algorithms assessing USPSTF criteria and major bleeding risks. Baseline data was collected for whether aspirin was algorithmically indicated (or not) for all patients at their first eligible primary care encounter in 20 clinics over 18 months. The analysis included patients age 18-75 (mean 58.4) with elevated cardiovascular disease risk (mean 10-year ASCVD risk 24.7\%) and excluded patients with congenital heart defects or diabetes. Aspirin overuse and underuse was determined by comparing concordance with: a) the algorithm's aspirin recommendation, and b) EHR-medication documentation of aspirin.

Results: Of the 11,682 patients meeting eligibility criteria at baseline, aspirin was indicated in 8,722 (74.7\%) and not indicated in 2,960 (25.3\%). Among patients with an aspirin indication, 6,493/8,722 (74.4\%) did not have aspirin documented (underuse). Among patients without an aspirin indication, 1,021/2,960 (34.4\%) had aspirin documented (overuse).

Conclusion: Overall, 7,514/11,682 (64.3\%) of patients who met study inclusion criteria for age and cardiovascular risk exhibited either potential overuse or underuse of aspirin for primary cardiovascular disease prevention. Despite expected missing documentation of aspirin due to its overthe-counter availability, which would result in measures of greater underuse and lower overuse than actuality, it is clear that patient aspirin use is very commonly inconsistent with USPSTF guidelines. The recommendation to consider colorectal benefits in the latest USPSTF draft could make decisions about aspirin appropriateness even more complex. EHR-based tools to help providers assess individualized risks and benefits of aspirin could greatly improve the quality of aspirin recommendations and potentially reduce costly cardiovascular disease events while simultaneously reducing rates of aspirin-related hazards.

Keywords: aspirin use, primary prevention
B1-4:

Leveraging Self-Report, Electronic Health Record and Human Resource Data to Estimate the Impact of Diabetes Mellitus on Worker Productivity

David C. Tabano, ${ }^{1}$ Arne L. Beck, ${ }^{1}$ Melissa L. Anderson, ${ }^{2}$ Paul A. Fishman, ${ }^{2}$ David C. Grossman, ${ }^{2}$ Debra P. Ritzwoller ${ }^{1}$

${ }^{1}$ Institute for Health Research, Kaiser Permanente Colorado, Denver, CO; ${ }^{2}$ Group Health Research Institute, Seattle, WA

Background/Aims: The economic burden of diabetes mellitus is high in the United States, increasing health care utilization and reducing workforce participation. Worker productivity is adversely impacted by complications related to diabetes. Estimating rates of absenteeism, presenteeism and other productivity measures can quantify the impact of diabetes in the U.S. workforce.

Methods: We used data from the Value-Based Benefit Design Health and Wellness Study Phase II (VBD), a multiyear prospective study of employees surveyed from Group Health Cooperative and Kaiser Permanente Colorado from Jan. 1, 2010, to Dec. 31, 2013. The VBD survey questionnaire includes self-reported data on physical activity at work and at home, the SF- $12^{\circledR}$ Health Survey, and self-reported data on absenteeism, presenteeism and lost productivity. These data were linked to the Virtual Data Warehouse (VDW) from both sites to determine whether respondents were diagnosed with diabetes. We applied the standardized relative resource cost algorithm to the VDW utilization data to calculate total health care costs per respondent.

Results: Across both sites 3,891 respondents to the baseline survey, all of whom provided consent to have their data included in the study, were analyzed. Of these, 286 (7.4\%) were identified as having diabetes. Compared to respondents without diabetes, respondents with diabetes had lower rates of positive overall health status ( $74 \%$ vs. $92 \%$ ), higher mean days worked while not feeling well (1.6 days vs. 0.9 days) and lower average physical health composite scale scores (46 vs. 52.6). Respondents with diabetes reported higher rates of presenteeism (1.1 vs. 0.7 hours per week), absenteeism (1.4 vs. 1.1 hours per week) and productivity due to illnesses (2.5 vs. 1.8 hours per week) compared to respondents without diabetes. Crude odds ratios for respondents with diabetes were higher for absenteeism $(1.35,95 \%$ confidence interval [CI]: 0.97-1.87), presenteeism (1.63, 95\% CI: $1.27-2.08)$ and lost productivity $(1.51,95 \%$ CI: $1.18-1.92)$. Average total health care costs were $\$ 1,222.98$ higher for respondents with diabetes.

Conclusion: Our preliminary results suggest employees with diabetes experience higher rates of presenteeism and lost productivity compared to employees without diabetes. Linking patient-reported outcomes to VDW and human resources data provides a detailed understanding of patient experience in the workforce.

Keywords: productivity, diabetes 
PS1-02:

Factors Associated With Longevity Among OldestOld Male Patients With Hip Fractures

Jinmyoung Cho, ${ }^{1,2}$ Eileen M. Stock, ${ }^{1,2}$ John E. Zeber, ${ }^{1,2}$ Brian K. Ahmedani, ${ }^{3}$ Rashmita Basu, ${ }^{1}$ Charlene C. Quinn, ${ }^{4}$ Laurel A. Copeland ${ }^{1,2}$

${ }^{1}$ Center for Applied Health Research, Baylor Scott \& White Health, Temple, TX; ${ }^{2}$ Texas A\&M Health Science Center, Round Rock, TX; ${ }^{3}$ Center for Health Policy and Health Services Research, Henry Ford Health System, Detroit, MI; ${ }^{4}$ University of Maryland School of Medicine, Baltimore, MD

Background/Aims: Few studies address the management and mortality of oldest-old male patients with hip fractures, although such events are increasing. This study identified factors associated with survival among male patients with surgically repaired hip fractures applying classification techniques to multiple chronic conditions (MCC), focusing on differences between patients $\geq 85$ years old and younger patients age $50-84$.

Methods: Data from the Veterans Health Administration identified 4,059 male patients age 50-100 with both hip fracture diagnosis and repair (index event) during fiscal years 2005-2009 (i.e. October 2004-September 2009). MCC was defined by Charlson and Elixhauser comorbidity conditions after eliminating overlapping diagnoses. A preliminary Cox regression model examined the association of MCC with survival. Latent class analysis produced classes using MCC, medications, use of physical therapy and demographic covariates (age, race/ethnicity, marital status, VA Priority denoting highly disabled). Cox proportional hazards modeled association between class and 5-year survival.

Results: The sample included 942 patients of age $\geq 85$ years $(23 \%)$. Survival censored at 5 years postoperation averaged 1,030 days, or almost 3 years. The oldest patients were more likely to be married ( $48 \%$ vs. $41 \%$ ) and to die within 6 months (36\% vs. $17 \%$ younger); minority race/ethnicity was similar, as was mean MCC (3.9 [SD: 2.8; median: 4.0] vs. 3.9 [SD: 2.7; median: 4.0]). Preoperative physical therapy was equivalent ( $24 \%$ vs. $24 \%$ ), but postoperative physical therapy was less common for those age $\geq 85(20 \%$ vs. $32 \%)$. Oldest patients also averaged fewer medication classes (5.1 vs. 5.5 preop; 6.4 vs. 7.4 postop). In a preliminary adjusted model, mortality risk increased by $13 \%$ per additional MCC (hazard ratio: $1.13,95 \%$ confidence interval: $1.11-1.15)$. After latent class analysis with three classes identified, $43 \%$ of oldest-old were classified primarily white and healthier, $43 \%$ white and sicker with more medication classes and $14 \%$ primarily black with alcohol abuse. Similar trends were observed for younger patients (36\%, 53\% and $11 \%$, respectively).

Conclusion: Although the oldest-old male population represents a relatively small proportion of patients, their longevity may be related to various health care factors. Close investigation of classification of individuals with MCC and hip fractures may help target interventions within health care settings and contribute to healthy aging.

Keywords: oldest-old male patients, chronic conditions
PS2-03:

Analysis of an Integrated Diabetes Care Program and Impact on Health Outcomes and Utilization

\author{
James M. Pitcavage, ${ }^{1}$ Wen Feng, ${ }^{1}$ Daniel D. Maeng ${ }^{1}$
}

${ }^{1}$ Center for Health Research, Geisinger Health System, Danville, PA

Background/Aims: For the past decade, Geisinger Health System has coordinated care for patients with diabetes using an integrated diabetes care model. This model utilizes the electronic health record to coordinate a program to improve physician adherence to nationally recommended diabetes measures. The aim of this study is to assess which patient level factors are associated with improved health outcomes for patients who receive care in this integrated diabetes program compared to patients with diabetes who receive nonintegrated diabetes care.

Methods: Study population consists of Geisinger Health Plan members with diabetes in the year 2013. Geisinger Health Plan is a private nonprofit insurance provider in Pennsylvania. The study examined 1,692 patients with diabetes (866 in the intervention group, 826 in the control group). This cross-sectional study uses survey data matched to administrative claims data to assess factors associated with health outcomes and costs. Chi-squared tests were used to determine whether there is a significant difference between patients with diabetes in an integrated program compared to patients in nonintegrated programs.

Results: Mean age of the study population was 67.8 years, and $45.4 \%$ were female. There were no significant differences between study cohorts regarding demographic characteristics including smoking and exercise habits. Subjects in the intervention group (i.e. received care in the integrated diabetes program) had statistically significantly higher scores for patient activation and for follow-up care coordination from their respective primary care provider. Subjects in the treatment group were more likely to feel that their doctor listens attentively to them $(\mathrm{P}=0.019)$ as well as more likely to be referred to a diabetes education class $(\mathrm{P}=0.001)$. There were no significant differences on emergency department use by each group. Cost results are pending.

Conclusion: Preliminary results show that patients who received care in the integrated diabetes care program score higher on patient activation measures as well as goal setting and follow-up coordination compared to patients who are not within the integrated diabetes program. Subsequent analyses will be conducted to study the relationship between outcomes and health care utilization.

Keywords: integrated care, diabetes 
PS2-04:

Human Resource Costs of Implementing a Tiered Team Care Model for Chronically III Patients According to Lean Management Principles

Laura Panattoni, ${ }^{1}$ Ellis C. Dillon, ${ }^{2}$ Lily Hurlimann, ${ }^{2}$ Meg Durbin, ${ }^{2}$ Ming Tai-Seale ${ }^{2}$

${ }^{1}$ Quality and Safety Initiatives, Qualis Health, Seattle, WA; 'Palo Alto Medical Foundation for Health Care, Research and Education, Palo Alto, CA

Background/Aims: The published literature on managing chronic conditions includes mostly randomized controlled trials, but is silent on implementation in real-world practice. We documented the Palo Alto Medical Foundation's (PAMF) human resource costs of developing and implementing a new chronic care model called Champion. Champion includes proactive outreach to patients with uncontrolled diabetes and hypertension, a redesigned primary care visit and health coaching. Champion aimed to improve patient and provider experience and outcomes, as well as cover costs, through expanding the role of nonlicensed personnel.

Methods: One clinic with 38 physicians serving 29,000 adult patients implemented Champion. We used an "activity-based costing" approach to investigate the organizational process. Activity-based costing divides indirect human resource costs, which dominate health system transformation, into specific activities. We searched the Microsoft Outlook calendars of key informants to determine time spent on Champion activities. Activity cost was calculated as the sum of hours spent by employees multiplied by their actual wages. The time period analyzed covered development, rollout and stabilization (July 2012 through May 2014).

Results: Implementation involved 162 employees - 30 managers, 46 physicians, 54 staff and 32 consultants - and cost $\$ 1,309,883$ (17,102 hours). Activities followed lean management principles: management guidance meetings, rapid process improvement workshops, value stream mapping, and final performance review. The 32 consultants were the most expensive employee-type: $\$ 402,190,6,343$ hours. The 54 staff were the least expensive: $\$ 262,210,6,183$ hours. The most expensive activity was the 15 employees who worked full time on implementation ( 3 managers, 5 consultants, 4 physician piloteers, 3 health coaching program staff). The health coaching staff worked 5,328 hours and cost $\$ 210,348$. Frontline provider trainings, which included all physicians and medical assistants, extended over 3 days $(2,352$ hours total) and cost $\$ 225,307$.

Conclusion: PAMF invested significant resources $(\$ 1,309,883)$ to Champion. The standard work developed is fundamental for future spread. PAMF may recover some of the implementation costs from additional revenues as physicians address unmet needs of chronic care patients. Champion's long-term financial sustainability should be evaluated as PAMF moves further into the era of accountable care, in which value of services brings more reward than volume of services.

Keywords: chronic conditions, cost
PS2-05:

The Burden of Hidradenitis Suppurativa in Maccabi Healthcare Services: Real-World Data Analysis

\author{
Dalit Litmanovitch-Goldstein, ${ }^{1}$ Vardda Shalev, ${ }^{1}$ Gabriel \\ Chodick $^{1}$
}

${ }^{1}$ Maccabi Institute for Health Services Research, Maccabi Healthcare Services, Tel-Aviv, Israel

Background/Aims: Hidradenitis suppurativa is a chronic, recurrent, inflammatory skin disease that presents with painful inflamed lesions in the apocrine gland-bearing areas of the body. Little is known on its medical and economic burden.

Methods: This retrospective cohort study was conducted in Maccabi Healthcare Services (MHS) using de-identified datasets. Included in this analysis were all MHS members with hidradenitis suppurativa diagnosed from Jan. 1, 2004, to Dec. 31, 2014. Data included sociodemographic details, chronic comorbid conditions and health care services utilization as well as prescriptions dispensed within 14 days after visits with hidradenitis suppurativa diagnosis.

Results: A total of 14,700 MHS members were detected to have at least one hidradenitis since 2004. Included in this study are 3,506 patients age 20 years and older with hidradenitis suppurativa (mean age: $38.5 \pm 12.8$; 63\% female). Among study patients, $25.4 \%$ were obese, $24.7 \%$ suffered depression and $35.3 \%$ were treated with antidepressive therapy. In 2013, $39 \%$ and $29 \%$ of the patients visited a dermatologist and a surgeon, respectively. Medications frequently prescribed and purchased are topical antibiotics, topical steroids combined with antibiotics and systemic antibiotics minocycline. Medical procedures most common among study patients were wound treatment, incision and drainage, both during first year after index hidradenitis suppurativa diagnosis and annually.

Conclusion: The present study is one of the first to use realworld data on the burden of hidradenitis suppurativa. Results indicate that hidradenitis suppurativa is a relatively common inflammatory skin disease in Israel with a considerable medical and economic burden.

Keywords: hidradenitis suppurativa, burden

\section{COMMUNICATION, DISSEMINATION, IMPLEMENTATION}

B5-1:

Large-Scale Implementation of Alcohol SBIRT in Adult Primary Care in an Integrated Health Care Delivery System: Lessons From the Field

Stacy A. Sterling, ${ }^{1}$ Thekla B. Ross,${ }^{1}$ Constance Weisner ${ }^{1}$

${ }^{1}$ Division of Research, Kaiser Permanente Northern California, Oakland, CA

Background/Aims: Screening, Brief Intervention, and Referral to Treatment (SBIRT) has been shown to be 
an efficacious intervention for risky drinkers and is recommended by numerous national organizations (NIH, USPSTF, SAMHSA, CDC). Nevertheless, large-scale implementation of SBIRT has proven to be challenging.

Methods: The roll-out of systematic adult alcohol SBIRT in Kaiser Permanente Northern California built on and scaled up the findings from a National Institute on Alcohol Abuse and Alcoholism-funded clusterrandomized implementation trial (ADVISe) of different modalities of SBIRT delivery - primary care provider (PCP)-delivered versus delivery by nonphysician provider. Based on findings from ADVISe, a hybrid SBIRT model was chosen for implementation in which evidence-based alcohol screening instruments and clinical decision support tools were developed for the health system's electronic health record, medical assistants were trained to screen all patients in adult medicine and PCPs were trained in brief intervention and referral to treatment techniques. Curricula and procedures were developed for medical assistant and PCP training, skills reinforcement and troubleshooting. Structures and protocols were created for communicating provider- and facility-level screening and brief intervention performance. We developed a network of "alcohol champions" across the health system within adult medicine and established collaborative relationships between medicine and chemical dependency programs to facilitate the seamless referral to and receipt of patients in specialty substance abuse treatment.

Results: Since the initial roll-out in July 2013, there have been 2,502,455 alcohol screenings on unique adult primary care patients; 3,575,039 total screenings have occurred including follow-up screenings for risky drinkers. The $86 \%$ regional rate of alcohol screening has exceeded tobacco and exercise screening. Brief intervention rates across the 15 medical centers in the region range from $31 \%$ to $71 \%$, with a current regional mean brief intervention rate of $54 \%$.

Conclusion: We will discuss the challenges to and critical stakeholder involvement in the successful largescale implementation of research findings and make recommendations for structures and approaches that may facilitate adoption of SBIRT within health systems.

Keywords: SBIRT, implementation

B5-2:

Evaluating Shared Decision-Making Across Clinical Topics in Primary Care: An Exploratory Study Using the OPTION ${ }^{5}$ Observer Measure

Ellis C. Dillon, ${ }^{1}$ Cheryl D. Stults, ${ }^{1}$ Caroline Wilson, ${ }^{1}$ Amy Meehan, ${ }^{1}$ Judith Chuang, ${ }^{1}$ Martina Li, ${ }^{1}$ Glyn Elwyn, ${ }^{2}$ Dominick Frosch, ${ }^{1}$ Ming Tai-Seale ${ }^{1}$

${ }^{1}$ Palo Alto Medical Foundation Research Institute, Palo Alto, CA; ${ }^{2}$ The Dartmouth Institute for Health Policy \& Clinical Practice, Hanover, $\mathrm{NH}$

Background/Aims: Shared decision-making (SDM) is vital in primary care because it is the most common setting for patients to seek medical care and is typically the first point of contact with health services. The recently developed OPTION $^{5}$ observer measure evaluates SDM during visits but has only been previously used to evaluate one topic per visit. In this study, we apply OPTION $^{5}$ to analyze the extent of SDM across the spectrum of topics presented in primary care visits. OPTION 5 allows observers to assess the following five items: how physicians 1) present options, 2) establish a partnership with the patient, 3) describe pros and cons of options, 4) elicit patient preferences, and 5) integrate patient preferences into the decision.

Methods: OPTION ${ }^{5}$ was used to evaluate SDM at four primary care clinics that are part of a large multispecialty group practice in northern California. Two researchers jointly identified "topics" requiring decisions, scored each item and then averaged across raters to create overall scores to indicate the level of SDM occurring for each topic. We used descriptive statistics and linear regression with clusterrobust standard errors to analyze the $\mathrm{OPTION}^{5}$ items in 40 audio-recorded visits. The models controlled for clinics and patient background characteristics.

Results: The 40 visits included 200 distinct topics (mean topics per visit: 5.03 , min: 1 , $\max : 13, \mathrm{SD}: 2.62)$. The mean overall OPTION $^{5}$ score was 27.2 (on a scale of $0-100$ ), and scores ranged from a minimum of 2.5 to a maximum score of 75 (SD: 15.15). Lower patient education levels were significantly associated with lower OPTION ${ }^{5}$ scores across all five items (item 1 coefficient: $-0.91, \mathrm{P}<0.05$; item 2 coefficient: $-1.68, \mathrm{P}<0.01$; item 3 coefficient: -2.04 , $\mathrm{P}<0.01$; item 4 coefficient: $-1.49, \mathrm{P}<0.01$; item 5 coefficient: $-1.36, \mathrm{P}<0.01$ ) and with the overall score (coefficient: -7.48 , $\mathrm{P}<0.001)$.

Conclusion: The relatively low overall scores suggest much room for improvement in SDM in primary care visits. The consistent association between patient education and SDM in visits suggests it is imperative to find methods to better engage patients with less education and that these patients should be a priority vulnerable population for SDM support and engagement strategies.

Keywords: shared decision-making, evaluation

B5-3:

Elements of Effective Noninvasive Ventilation Use in Patients With Chronic Obstructive Pulmonary Disease Among High-Performing Hospitals

Kimberly A. Fisher, ${ }^{1}$ Kathleen M. Mazor, ${ }^{1}$ Sarah Goff, ${ }^{2}$ Mihaela S. Stefan, ${ }^{2}$ Nicholas S. Hill, ${ }^{3}$ Michael B. Rothberg, ${ }^{4}$ Penny S. Pekow, ${ }^{2}$ Lauren A. Williams, ${ }^{2}$ Peter K. Lindenauer ${ }^{2}$

${ }^{1}$ Department of Medicine, University of Massachusetts Medical School, Worcester, MA; ${ }^{2}$ Center for Quality of Care Research, Baystate Medical Center, Springfield, MA; ${ }^{3}$ Division of Pulmonary and Critical Care Medicine, Tufts University School of Medicine, Boston, MA; ${ }^{4}$ Medicine Institute, Cleveland Clinic, Cleveland, $\mathrm{OH}$ 
Background/Aims: Noninvasive ventilation (NIV) is an important component of treatment for patients with respiratory failure due to exacerbations of chronic obstructive pulmonary disease (COPD). Proven benefits include reduced need for invasive ventilation, complications, mortality and hospital length of stay. Despite these wellestablished benefits and strong recommendations in clinical guidelines, utilization of NIV varies widely across hospitals. The purpose of this study was to identify approaches used by hospitals that have been successful in implementing NIV to treat respiratory failure among patients with COPD.

Methods: In-depth interviews were conducted with key stakeholders from a sample of high-performing hospitals selected from a large and representative network of 386 U.S. hospitals. High performers were defined as hospitals in which a high proportion of patients with COPD requiring mechanical ventilation were treated with NIV, and that achieved low risk-adjusted mortality. Interviews were audiorecorded and transcribed verbatim. Themes and subthemes were identified through iterative readings of the transcripts, generation of preliminary lists of themes and discussion among team members until the team reached consensus that all important themes and subthemes present in the first sample of 25 interviews had been identified.

Results: Interviews have been conducted with 25 participants from five hospitals, including respiratory therapists $(\mathrm{n}=12)$, physicians $(\mathrm{n}=8)$ and nurses $(\mathrm{n}=5)$. Preliminary analyses reveal three domains related to effective NIV use: 1) processes, 2) structural elements, and 3) contextual factors. Key processes included timely identification of appropriate patients and early initiation of NIV, frequent assessment of patient status and attention to patient comfort on NIV. Necessary structural elements included adequate equipment, sufficient numbers of qualified respiratory therapists and flexibility in staffing. Important contextual factors included achieving "buy-in" from key stakeholders, respiratory therapist autonomy, interdisciplinary teamwork and ongoing staff education. Hospital leaders, policies and protocols were identified as playing a supporting role in promoting essential elements.

Conclusion: These preliminary findings suggest elements of effective NIV use that characterize the experience of highperforming hospitals. By describing strategies to promote these elements, this study advances the understanding of how to successfully implement a complex intervention like NIV.

Keywords: chronic obstructive pulmonary disease, implementation science

B5-4:

Evidence-Based Mental Health Discussions During Periodic Health Exams: The Cup Is 1/3 Full

Ming Tai-Seale, ${ }^{1}$ Laura Hatfield, ${ }^{2}$ Caroline Wilson, ${ }^{1}$ Cheryl D. Stults, ${ }^{1}$ Thomas McGuire, ${ }^{2}$ Lisa Diamond, ${ }^{3}$ Richard Frankel, ${ }^{4}$ Lisa MacLean, ${ }^{5}$ Ashley Stone, ${ }^{1}$ Jennifer Elston Lafata ${ }^{6}$

${ }^{1} \mathrm{Pal}$ Alto Medical Foundation for Health Care, Research and Education, Palo Alto Medical Foundation Research
Institute, Palo Alto, CA; ${ }^{2} \mathrm{Health}$ Policy and Research, Harvard Medical School, Boston, MA; ${ }^{3}$ Memorial SloanKettering Cancer Center, New York, NY; ${ }^{4}$ Department of Medicine, Indiana University, Indianapolis, IN; ${ }^{5}$ Department of Psychiatry, Wayne State University, Detroit, MI; ${ }^{6}$ Social and Behavioral Health, Virginia Commonwealth University, Richmond, VA

Background/Aims: Periodic health examinations (PHEs) are the most common reason adults see a physician. The Affordable Care Act mandates one "free" PHE per person per year. We examine if there may be a potential value of PHE due to its role as a "safe portal" by which some patients seek mental health care. Methods: Study data came from audio recordings of 255 PHEs with patients likely to need mental health care. Coding the audio recordings and their transcripts enabled us to examine the timing of a mental health discussion (MHD), its quality and the relationship between MHD quality and physician practice styles, patient health and patient preparedness for the visit. The quality of MHD was measured by concordance with evidence-based practices as a three-level variable (evidencebased, perfunctory or none). Physician practice styles were measured from multiple visits of each physician with other patients: 1) length of visit; 2) talk time by the physician and the patient, which enabled us to measure the physician's verbal dominance; and 3) how fully the physician elicited the patient's agenda for the visit. We used electronic health records, patient surveys and direct observation to measure patient health status. The quantitative analysis used a generalized ordered logit model with partial proportional odds for the three-level ordinal dependent variable of MHD quality.

Results: Many patients came to their PHEs with mental health concerns, as over $50 \%$ of the MHDs occurred in the first 5 minutes of the visit. Only a third had an evidence-based MHD, another third had a perfunctory MHD, and the remaining third had no MHD. The analyses identified significant effects of physician practice styles. Visits with physicians who tend to spend more time with patients, fully elicit patient agendas and let patients talk (as opposed to being verbally dominant) were more likely to have a MHD.

Conclusion: If done well the PHE could be a safe portal for patients to raise mental health concerns, but most PHEs may not meet this standard. Improving the quality of PHEs may require reimbursement for longer visits, enabling physicians to more fully elicit patient agendas and to listen more attentively.

Keywords: patient-physician communication, mental health

PS1-29:

Beyond the Publishable Paper: Finding the Story in Your Research

Paige Backlund Jarquin, ${ }^{1}$ Charlene Barrientos Ortiz, ${ }^{2}$ Lisa Cuitto, ${ }^{3}$ Betty Hart, ${ }^{4}$ Crystal LoudHawk-Hedgepath, ${ }^{4}$ Lorenzo Ramirez, ${ }^{1}$ Curtis Robbins, ${ }^{5}$ Dee Smyth, ${ }^{1}$ Leslie Wright ${ }^{4}$

${ }^{1}$ Colorado Clinical \& Translational Research Institute, University of Colorado-Anschutz Medical Campus, 
Aurora, CO; ${ }^{2}$ Graduate School of Public Health, University of Colorado Denver, Denver, CO; ${ }^{3}$ Clinical Sciences Program, University of Colorado Denver, Denver, CO; ${ }^{4}$ Centers for American Indian and Alaska Native Health, University of Colorado-Anschutz Medical Campus, Aurora, CO; ${ }^{5}$ Kaiser Permanente Colorado, Englewood, CO

Background/Aims: "Beyond the Publishable Paper: Finding the Story in Your Research" was a workshop developed by the Colorado Clinical and Translational Sciences Institute Partnership of Academicians and Community for Translation to inspire the research community to think beyond journal publication as a means for translating and disseminating research findings. Aimed at attracting researchers, community research partners (present or in the future), health sciences students and trainees, the workshop objectives were to: a) demonstrate how the arts can be incorporated into the translation and dissemination process of research; b) identify elements of a good story; c) guide participants in creating a story from research results; d) identify strategies for sharing research results through storytelling; and e) practice performance art for storytelling. Methods: The 3-hour interactive, hands-on workshop taught participants the art of effective storytelling by kicking off with a performance of Loose Change, a research-based theatrical production developed from a Care Equity Project by Kaiser Permanente Colorado's Arts Integrated Resources Team. After learning about the elements of good storytelling, participants broke into groups and developed stories based on research findings in the two-page "Middle School Overview of 2013 Data" reported by Healthy Kids Colorado Survey. The workshop finale was a 5-minute performance by one of the groups illustrating the story they saw in the middle school data on "unintentional injury and violence."

Results: Participant postevaluation of the workshop was positive: a) The majority of respondents agreed or strongly agreed the workshop was a valuable use of their time (18 of 20); b) Most (15 of 20) thought the workshop was somewhat or very relevant to their work; and c) Holding the workshop in a community space, Su Teatro Cultural and Performing Arts Center, Denver, rather than on campus, supported participant learning and was worth additional effort to get there (17 of 20 respondents agreed or strongly agreed).

Conclusion: The workshop successfully modeled performance art as a tool for research dissemination, outlined a format for drawing stories out of data and provided hands-on practice in story development, theatrical performance and sharing.

Keywords: story, community-based participatory research

PS1-30:

Peer-to-Peer Toolkits Enhance Sharing of Best Practices Across an Integrated Delivery System

Randall C. Gale, ${ }^{1}$ Jeff Luck, ${ }^{2}$ Laura York, ${ }^{3}$ Steven Asch ${ }^{1,4}$

${ }^{1}$ Center for Innovation to Implementation, VA Palo Alto

Health Care System, Palo Alto, CA; ${ }^{2}$ College of Public
Health and Human Sciences, Oregon State University, Corvallis, OR; ${ }^{3}$ Center for the Study of Healthcare Provider Behavior, VA Greater Los Angeles Healthcare System, Los Angeles, CA; ${ }^{4}$ Division of General Medical Disciplines, Stanford University, Stanford, CA

Background/Aims: A peer-to-peer approach to develop and disseminate online packages ("toolkits") of locally developed system improvement resources ("tools") at Veterans Affairs (VA) medical centers across the country. Toolkits were launched between 2010 and 2015. Tools consisted of medical record-based templates, decision support resources, patient-tracking databases and service agreements intended to improve the quality and timeliness of care.

Methods: Series of online questionnaires and semistructured telephone interviews about tool use, targeting clinicians, quality managers and others involved in veteran care or quality improvement (QI).

Results: We evaluated uptake and use of the cancer/ palliative care, primary care, specialty care and emergency services for women toolkits. Since first toolkit launch in 2010, more than 30,000 unique individuals have visited with nearly 19,000 visitors to the primary care toolkit alone. We identified facilitators of toolkit awareness and use across all four titles, including: 1) presence of facility/ workgroup-level champions, 2) participation in learning collaboratives and the presence of other social networks, 3) engagement in QI initiatives, and 4) awareness of the facility's performance on quality metrics/reports. Among respondents to the cancer care toolkit questionnaire, those who were aware of their facility's performance on a VA-sponsored lung cancer study were significantly more likely to have visited the toolkit (odds ratio: 3.10 , 95\% confidence interval: 1.91-5.01). Respondents to the emergency services for women toolkit questionnaire who were involved in other QI activities were more likely to report toolkit use (odds ratio: 0.312 for uninvolved in QI, 95\% confidence interval: 0.14-0.69). Barriers to uptake included strained personnel and financial resources as well as complicated approval processes to support tool implementation.

Conclusion: User-driven online toolkits have broad appeal to providers and staff. They are a low-cost complement to other QI approaches, including gold standard toolkits whereby organizations rigorously design, test and disseminate tools. Additional work is needed identifying "less engaged" facilities and promoting toolkit use to them and others less closely tied to existing social networks. Increased opportunities for participation in learning/QI collaboratives and local support for the use of new tools may enhance uptake. Additional research is needed to increase our understanding of the link between toolkit use and clinical outcomes.

Keywords: quality, toolkit 
PS1-31:

Using the HCSRN Private Web Portal to Support Collaboration: Project-Based Experiences and User Insights

Beth Kirlin, ${ }^{1}$ Ella Thompson, ${ }^{1}$ Sarah Greene ${ }^{2}$

${ }^{1}$ Group Health Research Institute, Group Health Cooperative, Seattle, WA; ${ }^{2}$ Health Care Systems Research Network, Seattle, WA

Background/Aims: The Health Care Systems Research Network launched a password-secured web portal (HCSRN Portal) in October 2014 at www.hcsrn.org/share/. The HCSRN Portal allows project teams, scientific interest groups and committees to maintain online work and archival space, with access restricted to project-specific staff. The HCSRN Portal enables the manager of each private portal site to add or remove a variety of functions including a document library, calendar, wiki, blog, discussion board, data lists (e.g. action logs, contact directories, etc.) and quick links. To date, eight collaborative projects have established private project-specific sites within the HCSRN Portal. We describe the basic functionality available in the portal, functionality most used by project teams, and user experience and satisfaction.

Methods: We will survey individual users and site managers of the eight project-specific sites to assess their motivation for establishing a private site in the HCSRN Portal, perceived usefulness of the various portal functions, and perceived pros and cons of the portal overall as well as general user experience, degree of use, desired new or improved features and overall satisfaction with the portal.

Results: The survey is in progress; results are pending.

Conclusion: HCSRN leaders and project teams have desired a private web portal for many years. Now that this resource is available, an evaluation of its utility and general user experience could help enhance uptake and spread of the resource to other HCSRN teams in need of a system for securely sharing information across collaborating sites and identify potential improvements to support project-based use. Keywords: web portal, project communications

\section{PS1-32:}

Design Features of Successful Outpatient Chronic Disease Care Clinical Decision Support Systems

Patrick J. O'Connor, ${ }^{1}$ JoAnn M. Sperl-Hillen, ${ }^{1}$ Stephen E. Asche, ${ }^{1}$ A.L. Crain, ${ }^{1}$ Heidi L Ekstrom, ${ }^{1}$ Karen L. Margolis ${ }^{1}$

\section{${ }^{1}$ HealthPartners Institute for Education and Research,} Minneapolis, $M N$

Background/Aims: To identify key design features of pointof-care diabetes clinical decision support (CDS) that have high use rates and high provider satisfaction rates, and that have improved control of major cardiovascular risk factors.
Methods: Based on a series of National Institutes of Healthfunded projects to develop point-of-care electronic health record-linked, web-based CDS systems, we have identified design features that contribute to observed high use rates $(60-80 \%)$ at targeted visits, high primary care provider satisfaction rates (94-95\%) and positive effects on glucose and blood pressure control in adults with diabetes.

Results: The ideal outpatient chronic disease care CDS system would include the following features: a) co-designed by primary care physicians (PCPs) and researchers, b) supported by clinic and medical group leaders, c) designed to improve publicly reported quality measures, d) introspective identification of targeted encounters, e) total target encounters limited to about $20 \%$ of all adult visits, f) rooming nurse launches CDS early in encounter workflow, g) PCP sees CDS early in workflow and uses for visit planning, h) patient reviews CDS before PCP enters room, i) simple visual display of potential benefits for patients, $j$ ) prioritization of treatment options based on potential benefit to patient, k) automated feedback to PCP and clinics on CDS use rates at targeted encounters, 1) compensation to clinics to cover training costs, $\mathrm{m}$ ) location of algorithms in web service to facilitate updates and scalability, and n) built-in SmartSet to facilitate clinical actions.

Conclusion: These design features may inform future iterations of chronic disease CDS systems.

Keywords: clinical decision support, population health

PS1-33:

\section{Operationalizing a National Research Network}

Andrea D. Buchmeier, ${ }^{1}$ Christine Cameron, ${ }^{1}$ Krista Bradley, ${ }^{1}$ Jan Lowery ${ }^{1}$

${ }^{1}$ Center for Clinical Research, $\mathrm{CHI}$ Institute for Research and Innovation, Englewood, CO

Background/Aims: Catholic Health Initiatives (CHI) is one of the largest faith-based health care systems in America. In 2009, CHI established the CHI Institute for Research and Innovation (CIRI). CIRI is the research service line for CHI and works closely with $\mathrm{CHI}$ hospitals and physicians across the nationwide health system to: a) centralize administrative operations to reduce costs, b) provide training and standardize best practices to improve quality and compliance, c) increase community and physician awareness on the value of clinical research, and d) build research disease site portfolios to increase patient volumes.

Methods: The initial step in building a national research network is to establish a vision, which is then implemented through operationalizing many systematic steps to build a national network. Important stages include building the research team, the administrative infrastructure at the national level and the boots-on-the-ground clinical research staff at each local site. Standardization and streaming of processes are key to establishing a quality program and mitigating risk.

Results: The formation of CIRI was intended to improve 
the administrative management of clinical trials and reduce redundancy of functions at each site by centralizing and further streamlining these processes. One of the key results from this centralization has been a significant reduction in site research expenses; even with the addition of the centralized function expenses to the site expenses, total expense has been reduced from the original site expense by $40 \%$. This reflects that the centralized model has proven to be much more cost-effective than having multiple research sites operating independently.

Conclusion: Many lessons have been learned since the initial launch of the national network: a) National and local administrative champions are essential to success; b) Take time and build the network right — no shortcuts; c) Set SMART goals as envisioned by all stakeholders; d) Try to overcommunicate; e) Engage communities through outreach and education efforts; f) Engage industry and government partners early and often; g) Information technology infrastructure should include a clinical trial management system and website presence; and h) Standard operating procedures provide the foundation for standardization and quality monitoring.

Keywords: health services research, research administration

\section{GENETICS, GENOMICS, PRECISION MEDICINE}

\section{A5-1: \\ The Geisinger MyCode ${ }^{\circledR}$ Community Health Initiative: Experiences in Patient Recruitment, Genomic Sequencing, and Patient Engagement in a Population-Based Biobank}

\author{
Alanna Kulchak Rahm, ${ }^{1,2}$ Samantha Fetterolf,, Ryan \\ Colonie, ${ }^{2}$ Michael Murray, ${ }^{1}$ David J. Carey ${ }^{2}$
}

${ }^{1}$ Genomic Medicine Institute, Geisinger Health System, Danville, PA; ${ }^{2}$ Center for Health Research, Geisinger Health System, Danville, PA

Background/Aims: Geisinger's MyCode ${ }^{\circledR}$ Community Health Initiative is a population-based biorepository that began in 2007. Participants give broad consent for research use of their samples and electronic health record data, including genomic analysis, and permission to re-contact for additional studies. Participation in MyCode is open to all Geisinger patients, with a goal to enroll 250,000 participants. In 2013, consent was revised to allow for return of medically actionable information discovered through genomic studies to patients and providers and to include the information in the electronic health record. In 2014, a collaboration was initiated with the Regeneron Genetics Center to perform whole exome sequencing on biobank samples. The Governing Board approves all research conducted on biobank specimens, the Return of Results Oversight Committee monitors the return of clinical findings, and the External Ethics Advisory Committee provides independent feedback on MyCode operations and the clinical return of results process. Community members are engaged through research focus groups, newsletters and as members of the two committees.

Methods: MyCode is visible via signage throughout Geisinger clinics and system website. Approximately 20 MyCode consenters are stationed in outpatient clinics throughout the system. Samples are collected at the time of clinical blood draws. Consented participants who have not had clinical bloodwork may arrange to provide a MyCode sample if desired. Saliva collection may also be requested. In May 2015, an educational campaign was launched to ensure that all Geisinger employees can answer patient questions about biobank participation.

Results: At this time, approximately 1,000 patients consent per week, 90,000 patients have consented (85\% response rate), 55,000 have at least one sample in the biobank, and 50,000 exomes have been sequenced thus far. The population of consented patients is $97 \%$ white, $62 \%$ female and $50 \%$ aged 50-79 years.

Conclusion: Whole exome sequence information combined with patient medical record data is allowing Geisinger researchers and research partners to investigate new approaches to prevention, diagnosis and treatment of disease. Strong community engagement programs maintain trust in the system, keep participants engaged in research and continue to guide the evolution of the MyCode program.

Keywords: biorepository, genomic sequencing

A5-2:

\section{Parental Expectations and Attitudes Towards Receiving Genomic Information About Children Participating in a Population-Based Biorepository}

Alanna K. Rahm, ${ }^{1}$ Audrey Fan, ${ }^{1}$ Lindsay Bailey, ${ }^{1}$ Kara Fultz, ${ }^{1}$ Janet L. Williams, ${ }^{1}$ F. Daniel Davis ${ }^{2}$

${ }^{1}$ Genomic Medicine Institute, Geisinger Health System, Danville, PA; ${ }^{2 B}$ Bioethics, Geisinger Health System, Danville, $P A$

Background/Aims: Guidelines published by the American College of Genetics and Genomics recommend, when clinical sequencing studies are performed, returning genetic results in 56 genes, regardless of patient age. The American Academy of Pediatrics and standard clinical genetic testing practice recommend neither testing nor providing results to children for adult-onset conditions with no childhood manifestations or screening guidelines, such as hereditary breast and ovarian cancer (HBOC) and Lynch syndrome. Geisinger's MyCode ${ }^{\circledR}$ biorepository aims to collect samples and whole exome sequence data on 250,000 adult and pediatric participants. Consent to participate in MyCode includes consent to receive genomic results. Some genomic results have medical implications and management guidelines for children; however, the six genes related to HBOC and Lynch syndrome have no immediate health implications or recommended medical management for children. Prior studies have indicated that our adult 
participants are interested in genomic results regardless of medical actionability; however, few studies have reported on parental attitudes towards receiving results for their children that are medically actionable in childhood versus those that do not have implications until adulthood.

Methods: Multiple focus groups are being conducted with adult MyCode participants who have at least one child enrolled in the biorepository. Participants are selected on the basis of child age (0-8 or 9-17) and geographic location. A semi-structured guide was developed to elicit expectations regarding their children's participation in the biorepository and opinions and reactions to different return of results scenarios. Parents are first introduced to the issues through a scenario in which they learn that a child has a genetic condition that can be medically managed in childhood (Marfan syndrome), and then they are challenged to think about receiving genetic information about adult-onset conditions in their children, specifically HBOC and Lynch syndrome.

Results: Results from in-depth thematic analysis of focus group transcripts will be presented.

Conclusion: In developing pediatric biorepository consent processes and return of results protocols, it is crucial to elicit and understand both parental and, to the extent possible, pediatric participant attitudes and responses to different types of genomic information - specifically information that has childhood implications versus information that is medically actionable only during adulthood.

Keywords: whole exome sequencing, biorepository

A5-3:

Universal Screen for Lynch Syndrome in an Integrated Health Care System: Assessment of Patient Perspectives and Sharing Results With At-Risk Relatives

Jessica Ezzell Hunter, ${ }^{1}$ Jamilyn M. Zepp,, ${ }^{1}$ James V. Davis, ${ }^{1}$ Kellene M. Bergen, ${ }^{1}$ Kristin R. Muessig, ${ }^{1}$ Susan K. Peterson, ${ }^{2}$ Sapna Syngal, ${ }^{3}$ Louise S. Acheson, ${ }^{4}$ Georgia L. Wiesner, ${ }^{5}$ Jacob A. Reiss, ${ }^{1}$ Katrina A.B. Goddard ${ }^{1}$

${ }^{1}$ Center for Health Research, Kaiser Permanente Northwest, Portland, OR; '2Department of Behavioral Science, University of Texas MD Anderson Cancer Center, Houston, TX; ${ }^{3}$ Division of Medical Oncology, Department of Medicine, Dana-Farber Cancer Institute, Brigham and Women's Hospital, Harvard Medical School, Boston, MA; ${ }^{4}$ Departments of Family Medicine and Community Health and Reproductive Biology, Case Western Reserve University and University Hospitals Case Medical Center, Cleveland, OH; ${ }^{5}$ Hereditary Cancer Program, Department of Medicine, VanderbiltIngram Cancer Center, Vanderbilt University, Nashville, TN

Background/Aims: Lynch syndrome (LS) is the most common cause of hereditary colorectal cancer (CRC). Evidence-based recommendations promote universal tumor screening for LS among all new cases of CRC over selective screening based on family history or age of diagnosis. Maximal reduction in morbidity and mortality from universal tumor screening depends on patients with a positive screen following up with genetic counseling and testing to confirm a diagnosis of LS and sharing results with at-risk relatives.

Methods: Participants included 165 Kaiser Permanente Northwest members aged 39 to 91 years who had undergone surgery for CRC. Tumor samples were screened for microsatellite instability and participants surveyed before and after receiving screening results to assess perspectives on screening and sharing results with at-risk relatives.

Results: Most patients reported no family history of CRC; $14.1 \%$ had a first-degree relative and $7.4 \%$ had a secondor third-degree relative with CRC. However, most $(93 \%)$ wanted to know their risk for hereditary CRC. Overall, most patients endorsed potential benefits and few barriers to screening, though $62 \%$ indicated a worry about the cost of additional testing and surveillance. Before receiving screening results, most patients indicated they would likely share their result with their parents $(90 \%)$, siblings $(96 \%)$ and children (97\%), where applicable. Of the 25 patients with a positive microsatellite instability screen, $96.0 \%$ reported they shared their results with at least one relative. Most patients endorsed motivations to share results, namely: so family members could act to reduce their risk of CRC $(76 \%)$ and it was their responsibility to let family members know they might be at higher risk of CRC (68\%). None of the patients indicated that strained family relationships would prevent them from sharing results, and few indicated that they would not share their results because it would worry relative(s) $(8 \%)$ or because discussing their results could hurt relationship(s) (4\%).

Conclusion: Given universal tumor screening will target all new cases of CRC, it is important to understand motivations among patients not identified via high-risk factors such as family history or age of diagnosis. These findings provide insight into patient attitudes and perspectives toward LS screening to guide successful implementation of screening programs.

Keywords: Lynch syndrome, genetic screening

A5-4:

High-Risk Breast Cancer Clinic - A New RiskStratified, Evidence-Based, and Efficient Patient Care Model

Susan R. Snyder, ${ }^{1}$ Rosemary Leeming, ${ }^{2}$ Alanna K. Rahm, ${ }^{1}$ Jing Hao, ${ }^{1}$ James M. Pitcavage ${ }^{1}$

${ }^{1}$ Center for Health Research, Geisinger Health System, Danville, PA; ${ }^{2}$ Geisinger Medical Center, Geisinger Health System, Danville, PA

Background/Aims: There is considerable variation in adherence to available evidence-based recommendations for risk assessment, screening, genetic counseling and 
testing, surveillance and preventive care for women at risk for breast cancer. To improve adherence and patient outcomes and to facilitate appropriate referral to genetic testing, Geisinger implemented a new care model in February 2015 to systematically and efficiently deliver appropriate risk assessment and management services using midlevel providers. This "high-risk breast cancer clinic" provides any interested woman with a personal risk assessment and care plan. Validated risk assessment approaches are used to stratify women into differing risk levels. This new approach focuses on the large moderate-risk (5-year risk $\geq$ $1.7 \%$ and/or lifetime risk $\geq 20 \%$ ) and average-risk (5-year risk $\leq 1.7 \%$ ) populations, who comprise the majority of women and who are typically underserved with usual care. It provides them with evidence-based counseling, surveillance and chemoprevention care services. This clinic also serves women with dense breasts (density $>50 \%$, which includes about half of all women) who also are known to be at increased risk for breast cancer.

Methods: For women at moderate risk of developing breast cancer, a decision-analysis model compares care, outcomes and costs for the high-risk breast cancer clinic's approach to usual care. Assumptions for the model are based on Geisinger patient data, literature and expert opinion to develop estimates for clinical and economic outcomes.

Results: Adecision-analysis model comparing Geisinger's highrisk breast cancer clinic to usual care is used for a budget impact analysis to show the estimated effects of differences in patient care on outcomes and costs, including benefits of delivering riskbased prevention and treatment at earlier stages to moderate-risk patients. This analysis is used to make a business case for this new care model in an integrated health system.

Conclusion: A new targeted care model providing evidencebased evaluation and services to women at moderate and average risk for developing breast cancer offers the potential to improve delivery of appropriate care, patient experience and health outcomes with a favorable impact on cost.

Keywords: breast cancer, risk

PS-46:

Effects of Systematic Referral to Genetic Counseling
in High-Risk Women With and Without a College
Degree

Sarah Knerr, ${ }^{1,2}$ M. Robyn Andersen, ${ }^{3}$ Charles W. Drescher, ${ }^{3,4,5}$ Robert Resta, ${ }^{6,7}$ Michelle Hager, ${ }^{3}$ Carole Shaw, ${ }^{3}$ Kate Watabayashi, ${ }^{3}$ Nicole Urban ${ }^{1,3}$

${ }^{1}$ Department of Health Services, School of Public Health, University of Washington, Seattle, WA; ${ }^{2}$ Group Health Research Institute, Seattle, WA; ${ }^{3}$ Public Health Sciences, Fred Hutchinson Cancer Research Center, Seattle, WA; ${ }^{4}$ Pacific Gynecology Specialists, Seattle, WA; ${ }^{5}$ Obstetrics and Gynecology, School of Medicine, University of Washington, Seattle, WA; ${ }^{6}$ Swedish Cancer Institute, Swedish Medical Center, Seattle, WA; ${ }^{7}$ Hereditary Cancer Clinic, Swedish Medical Center, Seattle, WA
Background/Aims: Systematically identifying and referring women at high risk of hereditary breast and ovarian cancer to genetic counseling may minimize disparities in genetic services utilization. Our aims were to examine the effect of systematic referral on changes in testing-related cognitions and genetic services utilization and to test for effectmodification by education level.

Methods: In this secondary analysis of a randomized controlled trial, high-risk women $(n=625)$ were identified through electronic medical record data and mailed riskassessment questionnaires. Women that provided informed consent were randomized to either referral to genetic counseling $(n=228)$ or standard clinical care $(n=230)$.

Results: Referral was associated with a 78\% higher probability of participation in genetic counseling $(95 \%$ confidence interval: $72 \%-83 \%, \mathrm{P}<0.01$ ) and a $23 \%$ higher probability of undergoing genetic testing (95\% confidence interval: $16 \%-30 \%, \mathrm{P}<0.01)$ compared to standard clinical care. There was no evidence of differential intervention effects by education level, though exploratory analyses showed women with at least a college degree had larger increases in ovarian cancer relative risk perceptions from baseline to directly following genetic counseling.

Conclusion: Systematically identifying and referring highrisk women to genetic counseling using electronic medical record data and mailed risk-assessment questionnaires increased genetic services utilization, regardless of education level. Widespread dissemination of similar approaches may lead to more equitable genetic services delivery and improve cancer-related outcomes for high-risk women.

Keywords: genetic services utilization, health care disparities

\section{PS2-47:}

\section{Insurance Coverage Policies for Guideline- Recommended Genetic Testing for Targeted Cancer Therapies: Preliminary Results}

\author{
Christine Y. Lu, ${ }^{1}$ Rachel Ceccarelli, ${ }^{1}$ Kathy Mazoe ${ }^{2}$ \\ Ann $\mathrm{Wu}^{1}$
}

${ }^{1}$ Department of Population Medicine, Harvard Pilgrim Health Care, Boston, MA; ${ }^{2}$ Meyers Primary Care Institute, Worcester, MA

Background/Aims: Modern medicine is transitioning from empirical treatment to treatment on the basis of the underlying biology of the disease through the use of genomics-based technologies. Genomic tests are the fastest growing sector of medicine and medical science and have the potential to improve clinical practice. This study examined differences in patient access to guideline-recommended genetic tests that guide cancer treatment.

Methods: We reviewed publicly available coverage policies for 20 gene-cancer drug pairs (e.g. HER2, EFGR, BRAF tests), including 10 large private insurers and 12 Medicare contractors. We searched gene and drug names and key terms including gene, genomic and biomarker. We reviewed and extracted the following features: type of policy (gene- 
specific, drug-specific, generic policy for genetic testing); medical condition for which the test is covered; requirements for prior authorization; test methods and test result definition; and evidence basis for coverage.

Results: Across 10 private insurers, we identified 18 gene-specific policies, 63 drug-specific policies, 41 prior authorization requirements (for genetic test, drug or both) and 16 general policies for groups of genetic tests. Overall, some insurers have established gene-specific coverage policies that guide use of such tests, while most relied on policies guiding coverage of genetic testing more generally that may or may not include guideline-recommended genetic tests of interest. A few insurers only have drug coverage policies, and some of these did not recommend use of evidence-based genetic testing. Further analysis of policies is underway.

Conclusion: These preliminary findings of substantial variation in how insurers address guideline-recommended genetic tests underscore the need for additional research into the impact of these variations on cancer care and utilization. Keywords: pharmacogenomics, targeted cancer therapies

\section{PS2-48:}

Perspectives From Enrollees of a Large U.S. Health Care System on Informed Consent for Genetic Research

Monica M. Fuji, ${ }^{1}$ Lee Barr, ${ }^{1}$ Wylie Burke, ${ }^{2}$ Kenneth E. Thummel $^{3}$

${ }^{1}$ Group Health Research Institute, Seattle, WA; ${ }^{2}$ Department of Bioethics and Humanities, University of Washington, Seattle, WA; ${ }^{3}$ Department of Pharmaceutics, University of Washington, Seattle, WA

Background/Aims: To understand patient views about consent for secondary use and sharing of genetic data and specimens in the context of U.S. requirements for broad data sharing from the National Institutes of Health and the Department of Health and Human Services.

Methods: We surveyed 523 members of a large integrated health care system who previously participated in genetic research. We asked closed-ended questions about their perspectives on informed consent and biobanking.

Results: Of eligible participants, 273 completed the survey. Overall, sharing of genetic information and samples was acceptable in our participant population. Results about consent typeweremixed - no single type of consentwas overwhelmingly preferred. However, stratification by demographics showed that nonwhites, persons with higher income and men preferred study-specific over broad consent.

Conclusion: For most research participants, the broad sharing of information and biospecimens was acceptable. More work to better understand the consent preferences of populations by demographic factors may help in recruiting diverse study populations to generate more generalizable research results that benefit all members of society.

Keywords: human research subject protection, informed consent
ECl Awardee:

An Innovative Patient-Centered, Multidisciplinary Approach for the Delivery of Genomic Sequencing Results and Pharmacist-Led Lipid Management to Patients With Familial Hypercholesterolemia

Laney K. Jones, ${ }^{1}$ Eric A. Wright, ${ }^{2}$ Michael Evans, ${ }^{1}$ Mark S. Williams, ${ }^{3}$ Michael F. Murray ${ }^{3}$

${ }^{1}$ Enterprise Pharmacy, Geisinger Health System, Danville, PA; ${ }^{2}$ Center for Health Research, Geisinger Health System, PA; ${ }^{3}$ Genomic Medicine Institute, Geisinger Health System, Danville, PA

Background/Aims: Recent advances in genomic sequencing have provided new opportunities to identify individuals with familial hypercholesterolemia. The accessibility and usage of sequencing data for diagnosis and treatment of disease states has been scarce in medical practice but promises to be more widely available in the near future. Models for how to deliver genomic results to unsuspecting patients and provide postreturn care are needed. Our aim is to describe the first-ofits-kind multidisciplinary patient-centered model to deliver and provide care for patients with familial hypercholesterolemia identified through the MyCode ${ }^{\circledR}$ Community Health Initiative. Methods: At Geisinger the return of genomic sequencing results has been made possible through MyCode. The initiative began in 2006, and so far more than 50,000 patients have had exome sequencing completed. Over the next few years Geisinger aims to return results for certain actionable genetic conditions. An early-targeted population is those with familial hypercholesterolemia identified by analyzing exome sequence data obtained from MyCode participants. A review of current literature and internal care processes at Geisinger was conducted to determine the most appropriate delivery of care.

Results: Based on review of current literature and Geisinger care processes, there was substantial evidence to support a patient-centered multidisciplinary care team approach. Geisinger has previously seen excellent results in collaborative hyperlipidemia clinics. Our proposed multidisciplinary team will be comprised of two groups: 1) a core team (provider, pharmacist, dietician), and 2) an auxiliary team (cardiologist, primary care physician, obstetrician/gynecologist, genetic counselor). An initial visit will include a discussion of genetic results with patients, cascade testing of family members, baseline assessment, determination of patient-specific treatment goals and referral to cardiology if necessary. Subsequent visits will be led by clinical pharmacists and focus on maximizing familial hypercholesterolemia therapy, targeting treatment goals and responding to patient concerns. Initial qualitative feedback will be collected from team members and patients.

Conclusion: The development of this novel multidisciplinary team will allow us to provide patients with vital information about their health in a supportive environment. Future analysis will include a comparison of pharmacist-led team management to management provided by usual care on clinical, social and economic outcomes.

Keywords: familial hypercholesterolemia, patient-centered care 


\section{HEALTH DISPARITIES}

\section{C3-1: \\ Capturing Sex Partner Information in the Electronic Health Record: Implications for Research on Sexual Minorities Within an Integrated Health Care Delivery System}

Mark A. Schmidt, ${ }^{1}$ Alexandra M. Varga, ${ }^{1}$ Phillip M. Crawford, ${ }^{1}$ Sanchita Sengupta, ${ }^{1}$ Suzanne E. Gillespie, ${ }^{1}$ Judy L. Donald, ${ }^{1}$ Mary A. McBurnie ${ }^{1}$

${ }^{1}$ Center for Health Research, Kaiser Permanente Northwest, Portland, OR

Background/Aims: To better characterize and reduce health disparities faced by sexual and gender minorities (SGM), efforts to collect sexual orientation and gender identity within the electronic health record (EHR) are currently burgeoning across the United States. In 2006, Kaiser Permanente Northwest (KPNW) began collecting information about sexual activity of patients and the sex of their partners in the social history module of its EHR. The aims of our work were to describe trends in the capture of this information over a 9-year period and discuss the relevance of these findings to current sexual orientation and gender identity data collection and SGM health research efforts.

Methods: We included all KPNW members with at least one day of health plan enrollment and at least one in-person encounter (outpatient, inpatient or emergency department) from Jan. 1, 2006, to Dec. 31, 2014. We analyzed sexual activity and sex partner variables at encounter and individual levels over the study period.

Results: From 2006 to 2014, the proportion of encounters at which a patient's sexual activity was recorded increased from $1.2 \%$ to $15.1 \%$ for males and from $2.9 \%$ to $27.4 \%$ for females, with an increase among primary care encounters from $2.8 \%$ to $29.6 \%$ for males and $4.6 \%$ to $49.1 \%$ for females. Of our study population still enrolled in 2014, 37,890 males (22.1\%) had a sex partner recorded, $94.6 \%$ of which had female only, $5.1 \%$ male only and $0.3 \%$ both. At the same time, 87,851 females (40.7\%) had a sex partner recorded, $96.8 \%$ of which had male only, $2.8 \%$ female only and $0.4 \%$ both.

Conclusion: Our findings suggest an increasingly systematic capture of sexual activity and sex partner information within the KPNW EHR. Sexual behavior is only one component of sexuality orientation. As such, we are likely underestimating the size of our SGM population, especially for those reporting both male and female partners, based on previously published estimates. However, our research supports the idea that successful routine capture of sexual orientation and gender identity data within the EHR is possible. We argue that inclusion of these data would lead to a better understanding of the size and representativeness of the SGM population, more robust research studies and better interventions to reduce SGM health disparities.

Keywords: sexual orientation and gender identity, LGBT
C3-2:

A Qualitative Exploration of Perceived Health Issues Among Transgender Individuals in Atlanta and San Francisco

Ashli A. Owen-Smith, ${ }^{1}$ Cory Woodyatt, ${ }^{2}$ R. Craig Sineath, ${ }^{2}$ Enid Hunkeler, ${ }^{3}$ La Tasha Barnwell, ${ }^{4}$ Ashley Graham, ${ }^{1}$ Michael Goodman ${ }^{2}$

${ }^{1}$ Department of Health Management and Policy, Georgia State University, Atlanta, GA; ${ }^{2}$ Department of Epidemiology, Emory University, Atlanta, GA; ${ }^{3}$ Division of Research, Kaiser Permanente Northern California, Oakland, CA; ${ }^{4}$ Department of Behavioral Sciences and Health Education, Emory University, Atlanta, GA

Background/Aims: To date, health literature concerning lesbian, gay, bisexual and transgender (LGBT) persons has grouped these individuals into a single LGBT community. Lack of knowledge about transgender or gender-nonconforming ( $\mathrm{T} /$ GNC) individuals, specifically, precludes $\mathrm{T} / \mathrm{GNC}$ health from the public health agenda. To address this gap, we undertook a qualitative exploratory study to examine the perceptions of health issues among T/GNC individuals, including the perceived barriers to accessing health care and reliable information about health care services.

Methods: Data were drawn from 12 focus group discussions with 75 self-identified T/GNC men and women in Atlanta $(n=37)$ and San Francisco Bay Area $(n=38)$. Focus group discussions were stratified by gender and project site and were audio-recorded. The resulting transcripts were then reviewed for potential conceptual categories. Emergent themes were identified based on recurrence and on similarities/differences noted across the transcripts.

Results: Preliminary analyses suggest that T/GNC participants are most concerned about HIV/AIDS, mental illness related to trauma and depression, cancer risk and the long-term effects of hormone use. Participants reported experiencing many barriers to receiving timely and effective health care services. These barriers included: 1) poor access to affordable care (primarily due to cost/lack of health insurance coverage), 2) poor availability of culturally competent care, 3) perceived discrimination by health care providers, 4) difficulties navigating complex health care systems, and 5) lack of reliable information about transgender health care services. Though participants felt that the Internet can be a helpful resource for identifying information about providers and services, many participants expressed concerns that online information was not always accurate and was frequently confusing and inadequate. Word-of-mouth and social media resources were perceived to be most helpful. Additional analyses using MAXQDA software will be conducted prior to the conference.

Conclusion: Prior research demonstrates a deficiency in information regarding long-term health outcomes for $\mathrm{T} /$ GNC individuals. Our preliminary results indicate that $\mathrm{T} /$ GNC participants express concerns regarding the longterm effects of cross-sex hormones and surgery on chronic conditions like cancer, diabetes and cardiovascular disease. 
Additionally, despite recent advances in transgender care, many individuals experience significant barriers to accessing affordable, timely and culturally competent care and reliable information. Lessons learned from this study may inform future behavior-, health system- and policylevel interventions.

Keywords: transgender, health status

C3-3:

Perceptions of Barriers to and Facilitators of Participation in Transgender Health Research

Ashli A. Owen-Smith, ${ }^{1}$ Cory Woodyatt, ${ }^{2}$ R. Craig Sineath, ${ }^{2}$ Enid Hunkeler, ${ }^{3}$ La Tasha Barnwell, ${ }^{4}$ Ashley Graham, ${ }^{1}$ Michael Goodman ${ }^{2}$

${ }^{1}$ Department of Health Management and Policy, Georgia State University, Atlanta, GA; ${ }^{2}$ Department of Epidemiology, Emory University, Atlanta, GA; ${ }^{3}$ Division of Research, Kaiser Permanente Northern California, Oakland, CA; ${ }^{4}$ Department of Behavioral Sciences and Health Education, Emory University, Atlanta, GA

Background/Aims: Research regarding the health of transgender and gender-nonconforming (T/GNC) individuals and, moreover, methodological considerations for recruitment of this population, is scarce. As part of a larger effort to understand the feasibility of developing a cohort study to examine long-term health outcomes of hormonal therapy, we conducted focus group discussions to qualitatively explore attitudes toward health research involving T/GNC individuals. Specifically, we aimed to explore the potential facilitators and barriers to recruitment and enrollment and to identify points of influence that would aid future recruitment.

Methods: Data were drawn from 12 in-person focus group discussions with 75 self-identified T/GNC men and women in Atlanta $(n=37)$ and San Francisco Bay Area $(n=38)$. Discussions were stratified by gender and project site and were audio-recorded. The resulting transcripts were then reviewed for potential conceptual categories using the focus group discussion questions as initial categories. Emergent themes were identified based on both recurrence and similarities/differences noted across transcripts.

Results: Preliminary analyses suggest that individuals who identify as T/GNC experience several barriers to research participation. These include: 1) being unaware of the research, 2) having transportation and financial challenges, 3) believing that the research questions are irrelevant to the lives of T/GNC individuals, and 4) feeling distrustful of the medical and research community. Suggestions for how to improve research participation by individuals in the T/GNC community include: 1) recruiting in diverse communitybased locations using face-to-face referral/snowball-based methods; 2) scheduling research visits in locations accessible via public transportation and offering a variety of days/times to accommodate personal and professional responsibilities; 3) providing incentives including cash/gift cards, food and free/discounted health services; and 4) prioritizing transparency regarding the research questions and how the results will benefit the T/GNC community. Additional analyses of the transcripts using MAXQDA software will be conducted prior to the conference.

Conclusion: T/GNC individuals form a sizeable, unique and underserved community. Understanding the barriers and facilitators of successful enrollment and retention in research studies is critical to addressing health disparities experienced by this community. Results from these analyses will help to identify points of influence that would aid recruitment of T/GNC individuals into future observational studies and intervention trials.

Keywords: transgender, research participation

\section{C3-4:}

\section{Mobile Technology and Promoting Health Equity for Low-Income Patients}

\author{
Sharon S. Laing, ${ }^{1}$ Cindy P. Mendez-Hernandez, ${ }^{2}$ Carlota \\ Ocampo, ${ }^{3}$ Stacy Baugh ${ }^{3}$
}

${ }^{1}$ Nursing and Healthcare Leadership Programs, University of Washington Tacoma, Tacoma, WA; 'Women's Collective, Washington, DC; ${ }^{3}$ Trinity Washington University, Washington, DC

Background/Aims: Mobile health promotion (mHealth) is health practices supported by mobile devices like smartphones and wireless technology. mHealth delivers health information to increase awareness, provides guided instructions about healthy behaviors and offers self-monitoring of health status. Approximately $58 \%$ of adults and $43 \%$ of individuals earning less than $\$ 20,000 /$ year own smartphones; pervasiveness of mobile devices provides opportunity for patient selfcare management. A majority of low-income patients access services in community health centers (CHCs), thus an opportunity exists to evaluate how mobile devices can promote continuity of care in CHCs. The objectives are as follows: 1) Assess attitudes and practices concerning mobile technology; and 2) Evaluate how mobile devices can promote equity in health care access for low-income populations.

Methods: Researchers recruited a convenience sample of 103 low-income patients from CHCs in Washington State by approaching patients in waiting areas of two CHCs. After consenting, patients completed a 47-item survey in a private room and received a $\$ 10$ gift certificate. Survey items queried about: 1) knowledge and practices concerning mobile technology, and 2) perception about access to care in CHCs.

Results: Study population was $55.6 \%$ white, $67 \%$ women and had median household income of $\$ 21,192$. In terms of health care access, $80 \%$ and $83 \%$ reported easy access to specialists and health information, respectively. Approximately $89 \%$ emphasized the importance of making healthy lifestyle choices, yet only $56 \%$ and $66 \%$ received the CDC's recommended weekly physical activity and healthy eating guidance, respectively. In terms of technology knowledge and practices, 79\% used mobile devices for health information. 
Barriers to information access were: 1) lack of knowledge about use (52\%), and 2) preference for care providers' advice (42\%). Fifty-eight percent reported no knowledge about medical-based mobile apps (e.g. blood pressure monitoring), and $66 \%$ never used these devices. If medical-based apps were recommended, top preferred uses were: 1) prescription refills $(80 \%), 2)$ medication reminders $(66 \%)$, and 3$)$ mental health monitoring $(55 \%)$.

Conclusion: Some gaps in preventive care information dissemination do exist for patients receiving care in CHCs. However, patients appear ready to use technology in sophisticated ways for self-care management. Mobile devices might supplement care by providing health information and health status self-monitoring, thus helping to reduce health disparities.

Keywords: health care access, mobile technology

\section{PS2-49:}

Geographic Disparities in Influenza Vaccination Among Patients at Kaiser Permanente Mid-Atlantic States, 2014-2015

\author{
Alphonse J. Derus, ${ }^{1}$ Kevin B. Rubenstein, ${ }^{1}$ Suma \\ Vupputuri $^{1}$
}

${ }^{1}$ Mid-Atlantic Permanente Research Institute, Kaiser Permanente Mid-Atlantic States, Rockville, MD

Background/Aims: Yearly influenza epidemics can result in increased morbidity and mortality, absenteeism and productivity losses, and can overwhelm clinics and hospitals during peak illness periods. While safe and effective vaccines are available, disparities in influenza vaccinations rates are reported. The goal of this study is to examine geographic patterns of influenza vaccinations at Kaiser Permanente Mid-Atlantic States (KPMAS) (and assess the relative contribution of select covariates — data not presented here). Methods: We included members $\geq 18$ years old as of May 1, 2014, with continuous enrollment through May 31, 2015. Influenza vaccination was defined by diagnosis, procedure or medication codes, or documentation in the immunization table. Characteristics of those who did and did not receive the vaccine were reported. Vaccination rates were examined by county for Maryland (MD), District of Columbia (DC) and Virginia (VA). We will use the Blinder-Oaxaca decomposition method to determine what proportion of the geographic disparity could be reduced by equalizing select characteristics (data to be presented later).

Results: We identified 348,666 adult KPMAS members, of whom 141,238 (40.5\%) received the influenza vaccine. Vaccinated, compared to nonvaccinated, members were younger (mean age 55 [SD: 16.7] vs. 43 [SD: 16.2]), more likely to be white $(56,123[39.7 \%]$ vs. 49,435 [23.8\%]) and less likely to be African-American (47,028 [33.3\%] vs. 80,819 [39.0\%]). Education, mean distance to a medical center, body mass index and current smoking status were similar across the two groups. Vaccinated members were more likely to have had a primary care visit in the study year
$(126,677$ [89.7\%]) than nonvaccinated members $(134,011$ $[64.6 \%])$. Geographically, the lowest rates of influenza vaccinations $(<30 \%)$ were clustered in six adjacent counties located in DC and southern MD. Northern VA counties had the highest rates of vaccination. By race, vaccination rates were lower for African-Americans compared to whites in every county; however, a similar geographic pattern of low vaccination rates was observed in both races.

Conclusion: Among KPMAS members, a cluster of counties in DC and southern MD show the lowest rates of vaccinations. Examination of possible reasons for this disparity are needed. In subsequent analyses we will determine how specific characteristics contribute to the geographic disparity.

Keywords: influenza, disparities

PS-50:

Using Emergency Room Visitation Data to Identify Health Improvement Opportunities for Medicaid Enrollees

Scott B. Ode ${ }^{1}$

\section{${ }^{1}$ Medica Research Institute, Minnetonka, MN}

Background/Aims: Medicaid enrollees face many unique barriers when it comes to maintaining their health. Unfortunately, it is not always easy to identify these barriers and measure their consequences. One approach to understanding these disparities is to examine emergency room (ER) visitation patterns. By comparing ER utilization for individuals enrolled in Medicaid versus commercial insurance products, this study hopes to identify disparities in order to direct future research and shape public policy.

Methods: Claims for all ER visits in 2011 were obtained from a regional health plan for individuals enrolled in Medicaid or commercial fully insured products. Data from over 130,000 ER visits were captured. Diagnostic grouping software was used to determine the reason for each visit and visit frequencies for these groupings were calculated and compared across populations.

Results: The percentage of visits among Medicaid versus commercial enrollees was substantially larger for upper respiratory infections (URI; $10.3 \%$ vs. 5.9\%), otitis media (OM; $4.7 \%$ vs. $2.2 \%$ ) and disorders of the teeth and jaw (DTJ; 3.3\% vs. 1\%). Follow-up analysis examining these differences by age found that URI and OM accounted for the first and second highest disparity for individuals aged $0-12$ years (18\% vs. 14\% and $11 \%$ vs. $8 \%$, respectively), but this difference quickly dropped as age increased. Visits for DTJ were low among the $0-12$ age group $(<1 \%$ vs. $<1 \%)$ but were responsible for the largest visitation disparity for individuals age $13-25$ (1\% vs. $5 \%$ ) and 26-38 (2\% vs 6\%). DTJ accounted for the secondlargest disparity for individuals age 39-64 (4\% vs. 1\%).

Conclusion: A number of avenues for future research can be drawn from these findings, particularly those involving DTJ. Though patterns involving children may indicate environmental influences, they also may be a result of parents using the ER for primary care. Differences involving DTJ, however, are highly 
suggestive of unmet needs - most likely a mix of access to adequate dental care and treatment for drug addiction. Future research and policy implementation in this area will be crucial to improving the health and well-being of low-income populations. Keywords: emergency room utilization, dental care

\section{ECl Awardee: \\ Does Patient-Provider Racial/Ethnic and Language Concordance Impact Patient-Reported Experience of Care?}

Vani C. Nimbal, ${ }^{1}$ Beinan Zhao, ${ }^{1}$ Jia Pu, ${ }^{1}$ Robert J. Romanelli, ${ }^{1}$ Latha Palaniappan, ${ }^{2}$ Sukyung Chung ${ }^{1}$

${ }^{1}$ Palo Alto Medical Foundation Research Institute, Palo Alto, CA; ${ }^{2}$ Stanford University School of Medicine, Stanford, CA

Background/Aims: Patient-provider race/ethnicity or language (REL) concordance can influence quality of communication and thus patient experiences of care. We explored this relationship among the largest and fastest growing immigrant populations in the United States - Asian Indian, Chinese and Latinos compared to non-Hispanic whites (NHW).

Methods: Patient survey data (2013-2014) from the Consumer Assessment of Healthcare Providers and Systems (CAHPS) Clinician \& Group Survey were linked to electronic health records of patients in a large mixed-payer ambulatory care setting in northern California. We used multivariate logistic regressions to evaluate the effect of patient-provider REL concordance on patient experience of "Provider explanation understandable" (Yes, definitely vs. Yes, somewhat or No) and "Overall assessment of provider" ( 9 or $10 \mathrm{vs}$. $0-8$ on scale of 0 [worst possible] to 10 [best possible]) after controlling for other visit and provider characteristics influencing patient experience of care.

Results: Among 51,401 and 51,168 patients who provided answers to the two measures, respectively, 3\% were Asian Indians, 7\% Chinese, 8\% Latinos and 82\% NHW. Asian Indian and Chinese gave lower ratings for both outcomes compared to NHW, and patients with limited English proficiency gave lower ratings on "Provider explanation understandable" than English-proficient patients. Chinese providers were not rated differently from non-Chinese providers among both Chinese patients with and without limited English proficiency. Spanish-speaking Latinos gave lower ratings on "Overall assessment of provider" to non-Latino providers who speak Spanish (self-reported) (odds ratio: 0.17) compared to non-Latino providers who don't speak Spanish. Englishspeaking Latinos gave higher ratings on the measure to Latino providers (odds ratio: 2.54 ) than non-Latino providers. Conclusion: The impact of REL concordance on patient satisfaction is not uniform across racial/ethnic groups. Asians rate communication and overall assessment of providers lower than NHW in the same setting, and REL concordance did not improve their ratings. Latinos may prefer racial/ethnic concordant providers for better cultural sensitivity and a sense of shared beliefs and experiences, but Latinos with limited English proficiency may perceive a discrepancy between expectation and experience with language concordant-only providers. Greater diversity in demographics of health care professionals may not necessarily lead to improved patient experience unless truly culturally concordant care is provided. Keywords: race/ethnicity/language concordance, patient satisfaction

\section{HEALTH INFORMATICS, ANALYTICS, BIG DATA}

A3-1:

Predicting the Risk of Emergency Department Visits in Medicaid Members: Development and Temporal Validation of a Model

\author{
Eric S. Johnson, ${ }^{1}$ Xiuhai Yang, ${ }^{1}$ David H. Smith, ${ }^{1}$ Amanda \\ F. Petrik, ${ }^{1}$ Micah L. Thorp ${ }^{2}$
}

${ }^{1}$ Center for Health Research, Kaiser Permanente Northwest, Portland, OR; ${ }^{2}$ Northwest Permanente Medical Clinic, Kaiser Permanente Northwest, Portland, OR

Background/Aims: We developed and validated a model to predict the risk of emergency department (ED) visits in adult Medicaid members so that case-managers can identify the highest-risk patients and intervene. We then validated the model on newly enrolled Medicaid patients.

Methods: To develop the prediction model, we assembled a retrospective cohort of adult Medicaid members (1864 years old) enrolled at Kaiser Permanente Northwest between 2010 and 2013. We measured patient characteristics during the 90 days before the start of follow-up that might predict ED visits. We followed patients for up to 180 days to identify the first ED visit. To validate the model, we assembled a distinct cohort of adult Medicaid members who joined Kaiser Permanente Northwest in the first quarter of 2014. We developed and validated separate models for men and women using Cox regression.

Results: We observed 2,587 patients who visited the ED during the 180-day follow-up. The overall 180-day risk of an ED visit was 13.9 per 100 (men) and 17.4 per 100 (women). The models discriminated the high- and low-risk patients adequately: concordance or c-statistic was 0.72 (men) and 0.71 (women), respectively. The model's 10 predictor characteristics explained $35.2 \%$ of the variation in ED visits in men and $29.6 \%$ of the variation in women. Model calibration (agreement between observed and predicted) revealed that the mean predicted risks in the highest-risk patients underestimated the observed risks of an ED visit by approximately 11 per 100 . The model for women validated adequately in the newly enrolled cohort because the c-statistic remained constant while the model for men disappointed because the c-statistic dropped by 0.05 . For both men and women, the models continued to underestimate the absolute risk of an ED visit in the highest-risk patients.

Conclusion: The models identified the highest-risk patients with only 90 days of clinical history, and the models validated on new Medicaid patients. Time invested in managing the highest-risk patients may offer a superior return on investment 
compared with a strategy that does not stratify because the highest-risk patients suffer a disproportionate excess risk. The return on time invested may be even higher if recurrent ED visits are considered.

Keywords: prediction modeling, emergency department

\section{A3-2: \\ Integrating Technology Into Mental Health Intervention Trials}

Jennifer M. Boggs, ${ }^{1}$ Angelika D. Clarke, ${ }^{1}$ LeeAnn M. Rohm, ${ }^{1}$ Arne Beck, ${ }^{1}$ Rebecca Rossum, ${ }^{2}$ Julia Richards, ${ }^{3}$ Sona Dimidjian, ${ }^{4}$ Ursula Whiteside, ${ }^{5}$ Zindel Segal, ${ }^{6}$ Gregory Simon ${ }^{3}$

1 Institute for Health Research, Kaiser Permanente Colorado, Denver, CO; ${ }^{2}$ HealthPartners Institute for Education and Research, Minneapolis, MN; ${ }^{3}$ Group Health Research Institute, Seattle, WA; ${ }^{4}$ Department of Psychology, University of Colorado Boulder, Boulder, CO; ${ }^{5}$ School of Social Work, University of Washington, Seattle, WA; ${ }^{6}$ Department of Psychology, University of Toronto, Toronto, Canada

Background/Aims: Innovative web-based methods of delivering wide-scale mental health interventions in an efficient and sustainable manner have the potential to overcome barriers to care and increase access. Herein we describe lessons learned using an integrated informatics architecture developed to support implementation of two large randomized mental health pragmatic trials that are currently underway at three HCSRN sites. The Strategies for Overcoming Residual Depressive Symptoms (SOAR) study is evaluating the effectiveness of an online mindfulnessbased cognitive therapy (MBCT) program in 460 patients. The Suicide Prevention Outreach Trial (SPOT) is evaluating the effectiveness of two separate interventions - an online dialectical behavior therapy skills training program or risk assessment/care management using the Columbia Suicide Severity Rating Scale with secured messaging through Epic - in preventing suicide attempt/death in 16,000 patients. Each study integrates software tools for targeted patient outreach, retention and monitoring of suicide risk.

Methods: Both studies leverage real-time patient-reported outcomes data from Epic questionnaires for cohort identification and recruitment. Patients are then invited via email to visit websites to learn about the study and provide informed consent. REDCap, DatStat or Epic questionnaires are used to collect follow-up data from participants with real-time, item-level email alerts to notify study staff of suicide risk. An email service delivers study incentives to patients on survey completion. A dashboard was developed in Epic to facilitate population management for a large volume of high-risk patients.

Results: The integrated informatics architecture allows the study team to enroll patients efficiently and to maintain high patient retention with survey completion rates of $70 \%$ in the MBCT study at 6 months. We tested several approaches for suicide risk monitoring using web-based surveys to encourage patients' acceptance of subsequent outreach. Each HCSRN site adapted an Epic dashboard function that alerts care managers to high-risk patients and tracks appointment compliance.

Conclusion: Creative problem solving and effective partnerships among study staff, software and data programmers, care delivery teams, external collaborators and institutional review boards are essential for effective implementation of large pragmatic trials using multiple technologies. The integrated informatics architecture created here is essential to realize scalability, increase efficiency and support effectiveness of the interventions.

Keywords: technology, mental health

A3-3:

Visual Approaches to Bring Population Data Insights at Your Fingertips

\section{Ketan Mane, ${ }^{1}$ Jackie Blank, ${ }^{1}$ Michael Hornberg ${ }^{1}$ \\ ${ }^{1}$ Mid-Atlantic Permanente Research Institute, Kaiser Permanente Mid-Atlantic States, Rockville, MD}

Background/Aims: The data surge in electronic medical records offers a tremendous potential to leverage it to impact population/patient care delivery. However, to make sense of large data volume requires significant time commitment and attention - both of which are scarce resources for stakeholders in decision-making roles. Thus, a need exists to use technology that improves our ability to make faster inferences and decisions from data. At Kaiser Permanente Mid-Atlantic States, we are exploring novel ways to combine computational cycles and human intuitions in a learning loop to enhance care delivery experience. Our aim is simple: Instead of spending hours trying to gather information, we wanted to come up with a more user-intuitive approach that offers an easy way to sift through multidimensional data - to have the information right there at your fingertips for insights.

Methods: We focused on human immunodeficiency virus (HIV) cohort data from Kaiser Permanente Mid-Atlantic States. In contrast to our current approach of disseminating the report in tabular format, we adopted a visual data-driven strategy to best support user's tasks and to yield more information in less time. For rapid development, we used Tableau to build visual interface. From project inception, stakeholders were part of the design process to bring important perspectives. Data views that can be snapped together in a dashboard interface were built. Visual data encoding was used to reduce user's cognitive effort, and filters were added to help customize views to user's needs.

Results: The built HIV dashboard offered at a glance overview of population. Supporting interaction offered flexibility to instantly update data view to different inclusion/exclusion combinations as they came to user's mind. It offers decision makers macro-to-micro level population insights like: how the population is doing at the regional/service area/medical center level, how the population is distributed among different sites, and others.

Conclusion: The designed, visual, data-driven tool attempts 
to augment decision makers' cognition, support their ability to gather data insights and facilitate decision-making. Leveraging computational tools and designs, we show an approach to divide and conquer data, where the best of both human intuition and knowledge can be augmented by interaction with tools to boost performance.

Keywords: visualization, decision-making

\section{A3-4: \\ Data-Driven Modeling of Electronic Health Record Data to Predict Prediagnostic Heart Failure in Primary Care}

Kenney Ng, ${ }^{1}$ Walter F. Stewart, ${ }^{2}$ Christopher deFilippi, ${ }^{3}$ Sanjoy Dey, ${ }^{1}$ Roy J. Byrd, ${ }^{1}$ Steven R. Steinhubl, ${ }^{4}$ Zahra Daar, ${ }^{4}$ Heather Law, ${ }^{5}$ Alice R. Pressmnan, ${ }^{5}$ Jianying $\mathrm{Hu}^{1}$

${ }^{1}$ T.J. Watson Research Center, IBM Research, Yorktown Heights, NY; '2 Sutter Research Enterprise, Sutter Health, Walnut Creek, CA; ${ }^{3}$ University of Maryland School of Medicine, Baltimore, MD; ${ }^{4}$ Digital Medicine, Scripps Translational Science Institute, Geisinger Health System and Scripps Health, San Diego, CA; ${ }^{5}$ Research, Development, and Dissemination, Sutter Health, Walnut Creek, CA

Background/Aims: Electronic health records (EHRs) represent an opportunity for real-time early risk prediction. EHRs were used to determine the extent to which machine learning tools and a purely data-driven approach to modeling could detect heart failure (HF) subtypes, i.e. preserved ejection fraction $(\mathrm{HFpEF})$ and reduced ejection fraction (HFrEF), 12 months before a clinical diagnosis.

Methods: Incident HF cases were identified from Geisinger Clinic primary care patients aged 50-85 years, diagnosed between 2001 and 2010 and further defined as HFrEF if left ventricular ejection fraction $\leq 40$ and $\mathrm{HFpEF}$ if left ventricular ejection fraction $>50$. Controls were chosen to match HF cases by age, gender, location and primary care physician. EHR data were extracted on demographics, ICD-9 codes, medication orders, clinical and behavioral measures. Modeling was completed to detect $\mathrm{HF}$ using data from a 24-month observation window 12 months before the HF diagnosis. Patient feature vectors were generated from the data and summarized by one or more aggregation functions (e.g. counts, means). For the HF endpoint, modeling was done with and without patients who had an acute coronary syndrome event within 12 months of the diagnosis. Regularized logistic regression was applied using information gain feature selection and 10-fold cross validation. Model performance was assessed by area under the receiver operating characteristic curve and complexity by the number of selected features.

Results: Performance for HFpEF was better than for HFrEF. The HFpEF model was more complex than the HFrEF model as indicated by more EHR information that was needed to discriminate the HFpEF cases from controls. Performance with and without acute coronary syndrome cases was similar, though models including acute coronary syndrome cases were more complex than models excluding them.
Conclusion: Purely data-driven approaches to modeling can be used to detect HF 12 months before clinical diagnosis. Model performance and complexity varies with the HF subtypes, indicating differences in the complexity of modeling the HF subtypes.

Keywords: electronic health record, machine learning

PS2-51:

Physician Documentation Behaviors in Electronic Health Records as a Potential Source of Noise for Early Detection of Heart Failure

Sanjoy Dey, ${ }^{1}$ Kenney $\mathrm{Ng},{ }^{1}$ Jianying Hu, ${ }^{1}$ Roy J. Byrd, ${ }^{1}$ Steven R. Steinhubl, ${ }^{2}$ Christopher deFilippi, ${ }^{3}$ Alice R. Pressman, ${ }^{4}$ Walter F. Stewart ${ }^{4}$

${ }^{1}$ T.J. Watson Research Center, IBM Research, Yorktown Heights, NY; ${ }^{2}$ Digital Medicine, Scripps Translational Science Institute, Geisinger Health System and Scripps Health, San Diego, CA; ' University of Maryland School of Medicine, Baltimore, MD; ${ }^{4}$ Research, Development, and Dissemination, Sutter Health, Walnut Creek, CA

Background/Aims: Electronic health records (EHRs) are a potentially rich source of data for developing predictive models for early detection of heart failure. But, EHR data can vary because of both patient health and variation in clinical practice and behavior among physicians. The "noise" contributed by variable physician behaviors, such as differences in frequencies of documentation, could potentially confound predictive models for detection of heart failure. In this study, we characterized the documentation behaviors of primary care physicians (PCPs) in an effort to better understand this potential source of noise.

Methods: We used longitudinal EHR data on a stratified random sample of 5,187 patients who were: 1) 50 years of age or older, and 2) without a history of heart failure. PCPs $(\mathrm{N}=144)$ were identified and paired with the patients that they had treated for a minimum of 6 months. We derived 28 measures to characterize PCP behaviors - with documentation frequencies of assertions and denials of selected Framingham heart failure signs and symptoms (FHFSS) in office visit encounter notes adjusted for patient comorbidities. Hierarchical clustering analyses were performed on PCP documentation behaviors.

Results: PCPs were clustered into three groups with distinct documentation behaviors. Group 1 PCPs $(n=63)$ documented 10 out of 15 assertions and 11 of 13 denials of FHFSS, significantly more frequently than Group 3 PCPs $(n=20)$; Group 2 PCPs $(n=61)$ had significantly more frequent denial documentation behaviors than the other two. No significant differences were found among patients' chronic, episodic and cardiometabolic chronic disease counts (comorbidities) in each of the groups $(\mathrm{P}<0.05)$.

Conclusion: This study identified PCP groups with distinct documentation behaviors unrelated to patient complexity. This source of noise and potential confounder should be taken into account for predictive modeling.

Keywords: electronic health record, machine learning 
PS2-53:

Watson Health: A New Approach to Population Health and Research

Sharon Hensley Alford, ${ }^{1}$ John Piccone, ${ }^{1}$ Mark Sexton, ${ }^{1}$ Jason Gilder, ${ }^{1}$ Michele Pesanello ${ }^{1}$

\section{${ }^{1}$ Healthcare and Life Sciences, IBM Watson Health, Cambridge, MA}

Background/Aims: The population health management movement requires key strategies be employed by some of the nation's largest and most prestigious physician groups and health systems (most included in the HCSRN) to automate the management of their populations and improve health outcomes. Watson Health represents a health information technology (IT) and big data insights approach to enhance patient experience, management and outcomes. We bring together common data sources for evidence that is consistent across platforms, including payers, providers and pharmaceuticals.

Methods: Utilizing advanced analytics and IT structures, Watson Health data infrastructure management allows physicians and researchers to gain insights from existing data assets. Built on top of the enterprise data warehouse, interface strategies such as Explorys and Phytel have been built and instituted at several organizations. We have established trusted data for all stakeholders in the health care ecosystem to provide evidence that is needed for the collaboration between all aspects of health management and life sciences development.

Results: With the Explorys and Phytel tools, we were able to build tools to assist physicians and other care-team members. Data from registries, other providers, pharmacies, claims and laboratory sources was used to identify gaps in care that needed to be filled. Identification of specific patient populations can easily be identified for care alerts, clinical trial eligibility, research enrollment or follow-up at a huge time savings for the care team and research staff.

Conclusion: To create a sustainable health care system that provides affordable, high-quality health care to all, a population health management approach like that offered by Watson Health needs to be adopted by physicians and outcomes validated by health care research. Current electronic health records lack many of the features required to improve population health; therefore, new technologies are important to reach the overarching goal to improve population health.

Keywords: big data, population health

PS2-54:

\section{OpenNotes ${ }^{\circledR}$ - Building Relationships With Patients for Better Health and Health Care}

Arvind Ramaprasan, ${ }^{1}$ James D. Ralston, ${ }^{1}$ Robert Penfold ${ }^{1}$

${ }^{1}$ Group Health Research Institute, Seattle, WA

Background/Aims: OpenNotes ${ }^{\circledR}$ is a nationwide initiative supported by Robert Wood Johnson Foundation that offers patients ready access to progress notes of doctors and other clinicians after an office visit. In the original OpenNotes demonstration and evaluation project, patients were invited over email to read a note once it was signed by a primary care physician. The majority of patients in the study viewed notes and reported clinically relevant benefits. We know little about how many patients will view notes in the absence of a notification from a provider once the note is signed. The objective of this poster is to perform descriptive study on Epic/Clarity page views data and provide insights on how many notes are hidden and viewed using desktop/mobile devices.

Methods: We tracked all the face-to-face visits to the providers and associated notes data in the electronic health record (EHR) from April 10, 2015, to Sept. 10, 2015. We then linked EHR data to web server activity log based on medical record number and date to quantify notes accessed via desktop/mobile devices and mobile applications. We used Venn diagrams and Microsoft Excel graphs for visualization of results.

Results: Of the 679,111 outpatient visits during the period, $90 \%(610,625)$ had any associated note, $10 \%(68,486)$ did not, $96 \%(608,249)$ were accessible and $4 \%(2,376)$ were hidden from patient. Only $5.7 \%(34,780)$ of notes were viewed at least once during study period. Overall, there were 104,071 note views, i.e. 54,755 notes viewed at least once during the 5 months regardless of visit date, of which 19,975 notes were related to visits prior to April 10, 2015. We were able to match $104,019(99.95 \%)$ EHR data to web activity log data. There were $52(0.05 \%)$ unmatched records specific to Epic/Clarity alone. Web browsers accounted for $91.7 \%(95,473)$ of views [80.4\% (83,662) desktop, $11.35 \%$ (11,811) mobile], 4.7\% $(1,470)$ were AVS views on Group Health mobile application and $6.8 \%(7,076)$ were viewed on unknown device.

Conclusion: Desktop and mobile browsers continue to be popular platforms for viewing clinicians' notes. One of the reasons for low progress notes views is attributed to lack of active promotion on availability of EHR notes by Group Health Cooperation at that time. Group Health clinicians will continue to inform their patients that they can review the after-visit notes online.

Keywords: progress notes, after visit summary

PS2-56:

Tracking Health Care Team Response to Electronic Health Record Asynchronous Alerts: Role of InBasket Message Burden

Laura M. Sandford, ${ }^{1}$ Hassan Fouayzi, ${ }^{1}$ Devi Sundaresan, ${ }^{2}$ Jerry H. Gurwitz, ${ }^{3}$ Terry S. Field, ${ }^{4}$ Kathy M. Mazor, ${ }^{4}$ Lawrence Garber ${ }^{5}$

${ }^{1}$ Meyers Primary Care Institute, University of Massachusetts Medical School, Worcester, MA; ${ }^{2}$ Research Department, Reliant Medical Group, Worcester, MA; ${ }^{3}$ Division of Geriatric Medicine, University of Massachusetts Medical School, Worcester, MA; ${ }^{4}$ Department of Medicine, University of Massachusetts Medical School, Worcester, MA; ${ }^{5}$ Clinical Informatics, Reliant Medical Group, Worcester, MA 
Background/Aims: Electronic health record (EHR)based messaging allows communication of important patient information to primary care providers, but high message burdens may limit effectiveness and safety of this communication mode.

Methods: We analyzed EHR data for 1,282 discharges of elderly patients (age $\geq 65$ ) from a Massachusetts hospital in 2010-2011. For 799 of these discharges, time-sensitive automated EHR messages were generated on Day 3 postdischarge. These messages highlighted actionable concerns associated with medication changes and were sent to secure EHR In-Baskets belonging to the patients' primary care physicians. We tracked timing of message reviews and calculated associations between primary care physician InBasket message burden (i.e. number of messages in In-Basket at time of message delivery) and time to message opening. We also examined whether messages were opened by the intended physician, by a covering physician or by staff members.

Results: Of the 799 messages, 376 (47.1\%) were opened by the intended primary care physician within one business day, with an additional $2.0 \%$ (16) opened by covering physicians. In some cases, staff members opened messages and generated follow-up communication. These follow-up communications were read by the intended provider $(2.8 \%, \mathrm{n}=22$ messages $)$ or a covering provider $(0.9 \%, \mathrm{n}=7)$ within 24 hours of delivery. Overall, $41.1 \%$ of these time-sensitive messages were not opened by anyone within 24 hours; an additional $6.3 \%$ were opened by staff within the first 24 hours with no evidence that communications were passed along within the EHR. Rates of opening within 24 hours were inversely related to size of In-Basket at the time of message delivery, with $61.8 \%$ of messages opened within 24 hours among providers whose total number of In-Basket messages were in the bottom quartile (157 messages) $(\mathrm{P}<0.0001)$.

Conclusion: Rates of opening of time-sensitive In-Basket messages were suboptimal; less than two-thirds of messages were opened within 24 hours. In-Basket message burden may impact physicians' ability to devote timely attention to patient-related messages. Reducing In-Basket volume should be explored as a means of improving quality and safety of patient care.

Keywords: electronic health records, health care communication

\section{PS2-57:}

\section{Geographical Displays of Health Care Data}

\section{Christopher D. Mack ${ }^{1}$}

\section{${ }^{1}$ Group Health Research Institute, Seattle, WA}

Background/Aims: The HCSRN Virtual Data Warehouse has geocoded data on all of its members and associated data from the U.S. Census and American Community Survey. We wish to show several novel ways of using that information to benefit health care research.

Methods: We will be using SAS software data from our Virtual Data Warehouse, geographic information systems
(GIS) software and scripting languages to display existing data in ways that elucidate patterns of health care utilization and health outcomes that would otherwise be difficult to visualize. Results: Results of this study are pending.

Conclusion: Our project will determine whether geographical displays of data can be an important way to show data relationships and visualize those relationships in the clearest and most meaningful manner.

Keywords: geography, Virtual Data Warehouse

PS2-58:

Sulfonamide Use During the First Trimester of Pregnancy and Risk of Selected Congenital Anomalies Among Live Births

Craig Hansen, ${ }^{1}$ Susan E. Adrade, ${ }^{2}$ Heather Freiman, ${ }^{3}$ Sascha Dublin, ${ }^{4}$ Katie Haffenreffer, ${ }^{5}$ William O. Cooper, ${ }^{6}$ Craig Cheetham, ${ }^{7}$ Darren Toh, ${ }^{5}$ De-Kun Li, ${ }^{8}$ Marsha A. Raebel, ${ }^{9}$ Jennifer L. Kuntz, ${ }^{10}$ Nancy Perrin ${ }^{10}$

${ }^{1}$ South Australian Health and Medical Research Institute, Adelaide, Australia; ${ }^{2}$ Meyers Primary Care Institute, University of Massachusetts Medical School, Worcester, MA; ${ }^{3}$ Department of Emergency Medicine, Emory University School of Medicine, Atlanta, GA; ${ }^{4}$ Group Health Research Institute, Seattle, WA; ${ }^{5}$ Department of Population Medicine, Harvard Pilgrim Health Care, Boston, MA; ${ }^{6}$ Departments of Pediatrics and Health Policy, Vanderbilt University School of Medicine, Nashville, TN; 'Department of Research \& Evaluation, Kaiser Permanente Southern California, Pasadena, CA; ${ }^{8}$ Division of Research, Kaiser Permanente Northern California, Oakland, CA; 9 Institute for Health Research, Kaiser Permanente Colorado, Denver, CO; ${ }^{10}$ Institute for Health Research, Kaiser Permanente Northwest, Portland, OR

Background/Aims: Sulfonamide antibacterials are widely used in pregnancy, but evidence about their safety is mixed. Our aim was to assess the association between first-trimester sulfonamide exposure and risk of specific congenital malformations.

Methods: This retrospective cohort study included 1.2 million live born deliveries between 2001 and 2008 at 11 U.S. health plans participating in the Medication Exposure in Pregnancy Risk Evaluation Program, which has linked health plan with birth certificate data. Deliveries to mothers with first-trimester sulfonamide exposure were randomly matched 1:1 to those exposed to penicillins or cephalosporins (primary comparator) or no antibacterials (secondary comparator). We examined anomalies that in prior studies were associated with sulfonamide use: cardiovascular abnormalities, cleft lip/ palate, clubfoot, neural tube defects (NTDs), and urinary tract abnormalities. Outcomes were validated by medical record review. Analyses used conditional logistic regression.

Results: There were 6,688 deliveries in each exposure group included in primary analyses. Cardiovascular defects occurred in $1.6 \%$, urinary system defects in $0.7 \%$, clubfoot in $0.2 \%$ 
and cleft lip/palate in $0.1 \%$. NTDs were too rare for further evaluation $(\mathrm{n}=5)$. Sulfonamide exposure was not associated with significantly elevated risks for these anomalies. The adjusted odds ratio (OR) for cardiovascular defects was 0.94 (95\% confidence interval: $0.71-1.23$ ) for sulfonamide exposure compared to penicillin/cephalosporin exposure. The comparable odds ratios were $0.83(0.18-3.88)$ for cleft lip/ palate and $1.08(0.70-1.65)$ for urinary defects. For clubfoot, the odds ratio was elevated but not statistically significant (1.58 [0.71-3.50]). In comparison, analyses comparing sulfonamide exposure to no antibiotic use yielded estimates that were further from the null though still statistically nonsignificant.

Conclusion: First-trimester sulfonamide exposure was not associated with a higher risk of the congenital anomalies studied compared to exposure to penicillins or cephalosporins. Our results highlight the need to use active comparator groups exposed to medications with the same indication to minimize confounding.

Keywords: pregnancy, medication safety

\section{PS2-59:}

Division of Research Data-on-Demand: The
Development Process for a Research Data
Warehouse Rapid-Querying Tool

\section{Daniel Ng, ${ }^{1}$ Dimitri Buzkov, ${ }^{1}$ Srivardhan Chimmula, ${ }^{1}$} Jamila Gul ${ }^{1}$

\section{${ }^{1}$ Division of Research, Kaiser Permanente Northern California, Oakland, CA}

Background/Aims: Even simple Virtual Data Warehouse (VDW) queries require writing a program in SAS (or other software). While this is a necessary part of a study's overall analytical effort, investigators often need answers to basic questions prior to undertaking a formal study to develop hypotheses, estimate the population for a potential study, and ascertain other "what if" scenarios. Engaging an analyst can slow down the researcher's initial explorations and distract the analyst from more complex duties. A means for researchers to run their own simple VDW queries can enhance the efficiency of both researchers and analysts and contribute to faster, higher-quality research.

Methods: The DOR Strategic Programming Group engaged in a process that took over a year to develop a VDW rapidquerying tool for researchers. This involved a process of gathering user requirements, development of technical requirements, assessment of technology alternatives, creation of conceptual solution architecture, logical data model and physical solution architecture, interface design, security design, user training, user beta testing and enterprise security assessment.

Results: The result of this development process was the successful implementation of DOR Data-on-Demand (D3) within the Division of Research. D3 has nearly three dozen active users. Based on user testing and feedback, D3 has been enhanced with additional data domains beyond HCSRN's
VDW data model, including custom metadata (e.g. text description search for diagnoses and procedures, a custom medication list), medication orders, vaccine data and disease registry data. D3 also has been adopted by medical group analysts to provide rapid response to urgent research questions impacting clinical operations and quality of care. We continue to explore other ways that such a rapid-querying tool can be used to improve the health of Kaiser Permanente Northern California members.

Conclusion: The creation of D3 has given researchers the means to create and run their own rapid VDW queries. In addition, research analysts and data managers have found it useful for running more complex queries, validating data and improving data quality. Beyond research, it can enhance the analytic capabilities of quality and operations support groups. Finally, it can potentially assist clinicians in providing better evidence-based care.

Keywords: Virtual Data Warehouse, analytics

\section{PS2-77: \\ Use and Customization of Risk Scores for Predicting Cardiovascular Events Using Electronic Health Record Data}

Patrick J. O'Connor, ${ }^{1}$ Julian Wolfson, ${ }^{2}$ David Vock, ${ }^{2}$ Sunayan Bandyopadhyay, ${ }^{3}$ Gabriela Vazquez Benitez, ${ }^{1}$ Paul Johnson, ${ }^{4}$ Gediminas Adomavicius ${ }^{4}$

${ }^{1}$ HealthPartners Institute for Education and Research, Minneapolis, MN; ${ }^{2}$ Division of Biostatistics, School of Public Health, University of Minnesota, Minneapolis, $M N ;{ }^{3}$ Department of Computer Science and Engineering, University of Minnesota, Minneapolis, MN; ${ }^{4}$ Department of Information and Decision Sciences, Carlson School of Management, University of Minnesota, Minneapolis, MN

Background/Aims: The Framingham Risk Score (FRS) and the ACC/AHA Pooled Cohort Score (PCS) are widely used in clinical practice to guide individual patient care decisions. However, these risk scores have been estimated and validated mostly using data from longitudinal cohort studies; their performance when applied to patient data extracted from electronic health records is less well-established.

Methods: Risk factor data were obtained from the electronic medical record and insurance claims of 84,116 adults receiving care at a large health care delivery and insurance organization from 2001 to 2011. We assessed calibration and discrimination for four risk scores: the published versions FRS and PCS, and versions obtained by refitting the FRS and PCS using Cox regression models. Population subgroups in which the various models gave highly divergent risk predictions were identified using recursive partitioning techniques.

Results: The original FRS was well-calibrated (calibration statistic $K=7.4)$, but the original PCS was not $(K=39)$. Discrimination was similar in both models $(\mathrm{C}$-index $\mathrm{C}=0.740$ vs. $\mathrm{C}=0.747$ for original FRS and PCS). The refitted FRS $(\mathrm{K}=4.6, \mathrm{C}=0.754)$ yielded better calibration and discrimination than the original FRS; the refitted PCS $(\mathrm{K}=15.1, \mathrm{C}=0.746)$ 
was better calibrated than the original PCS. Individual risk predictions differed between original and refitted models for some subgroups, but were similar when comparing refitted models.

Conclusion: Both the FRS and PCS are appropriate for use in clinical decision support systems that rely on electronic health data, though it may be advisable to refit the models they are based on using available data from the target population to optimize performance.

Keywords: cardiovascular risk, risk prediction

\section{HEALTH SERVICES, HEALTH POLICY, COST}

\section{A2-1: \\ Assessing the Capacity of Primary Care Providers to Care for Vulnerable Populations After the Affordable Care Act Using Geoanalytical Spatial Mapping in a Large Hospital System in Northern California}

\author{
Maria R. Moreno, ${ }^{1}$ Dale Ainsworth ${ }^{2}$
}

${ }^{1}$ Research, Development, and Dissemination, Sutter Health, San Francisco, CA; ${ }^{2}$ California State University, Sacramento, CA

Background/Aims: The Affordable Care Act (ACA) was enacted to provide increased health care access to uninsured populations, many of whom represent the most vulnerable communities. Health insurance coverage does not automatically equate to access to health care. Understanding where capacity shortages are most pronounced, especially for low-income residents, gives community benefit professionals tools to make impactful, strategic decisions to facilitate the expansion of capacity in the most underserved communities.

Methods: Total number of Medi-Cal beneficiaries for Sutter's 23 counties pre- and post-ACA was estimated using California Department of Health Care Services state statistics. Total number of primary care physicians (PCP) was obtained from an online database through a customized search of primary specialties that included family medicine, general practice, internal medicine, obstetrics and gynecology, and pediatrics. Each physician was researched to establish accepted insurance types and eligibility criteria. We compared the distribution of PCP capacity and Medi-Cal populations at the ZIP code level using the allocation tools for ArcGIS geoprocessing toolbox with ESRI's ArcGIS 10.1 geographic information systems (GIS) software and the Network Analyst extension.

Results: Approximately $36.5 \%$ of the uninsured population became Medi-Cal eligible following ACA implementation for the Sutter communities. This location/allocation model identified the number of Medi-Cal patients per ZIP code that could not be assigned to any PCP because of the capacity limit for any PCP that was both within the county and within a 30-minute drive time. A series of maps were made that show the number of underserved patients within a 30-, 45and 60-minute drive time of each of Sutter's 24 hospitals. There is a considerable clinician shortage and a substantial number of people who do not have a medical home postACA. Within Sutter Health's 23 counties, approximately 2.1 million residents will be enrolled in Medi-Cal post-ACA, with approximately $610,000(29 \%)$ of these without access to a PCP.

Conclusion: While many Northern California communities have sufficient primary care, others have substantial shortages. Among nationally high emergency room usage for low acuity care, results of this analysis are being used by Sutter Health to facilitate the expansion of primary care capacity into these highly vulnerable communities.

Keywords: Affordable Care Act, GIS mapping

A2-2:

Who Are the Users of Medicare's New Preventive Visits Under the Affordable Care Act?

\section{Sukyung Chang, ${ }^{1}$ Jia Pu, ${ }^{1}$ Robert Romanelli, ${ }^{1}$ Hal Luft ${ }^{1}$ \\ ${ }^{1}$ Palo Alto Medical Foundation Research Institute, Palo Alto Medical Foundation for Health Care, Research and Education, Palo Alto, CA}

Background/Aims: We found previously that under the Affordable Care Act's new Medicare preventive visit coverage, called "Annual Wellness Visit," preventive visits among Medicare fee-for-service (FFS) beneficiaries nearly doubled from $18 \%$ to $34 \%$. The new coverage, however, may have differentially impacted subgroups of seniors. We hypothesized that preventive visit uptake would be greater for seniors who previously had frequent primary care visits, as they may have been "squeezing" preventive issues into office visits for acute and chronic care needs.

Methods: Medicare FFS beneficiaries aged 65-75 years who were primary care patients in a large, mixed-payer outpatient organization in northern California were studied. We examined patient characteristics associated with increased preventive visits between the period prior to (2007-2010) versus post (2011-2013) Medicare's coverage expansion. The unit of analysis was patient-years $(\mathrm{N}=112,888)$. Logistic regression was used with a dependent variable of "any preventive visit," including both Medicare-covered and any privately paid preventive visits. Random effects model was used to account for multiple observations per patient. To identify subgroups of seniors who were more likely affected by the coverage expansion, we used interactions between "post period" and key patient characteristics: age 70-75 (vs. 65-69), frequency of nonpreventive primary care visits $(1,2$, or $3+$ vs. 0$)$, and comorbidity by Charlson index ( 1 or $2+$ vs. 0$)$.

Results: Older seniors (odds ratio [OR]: 0.57 for age 70-75 vs. 65-69), males (OR: 0.92), and those who previously made frequent primary care visits (OR: 0.18 for 1 vs. 0 ; OR: 0.13 for 2 vs. 0 ; OR: 0.11 for $3+$ vs. 0 ) and had more comorbidities (OR: 0.80 for 1 vs. 0 ; OR: 0.78 for 2 vs. 0 ; OR: 0.51 for $3+$ vs. 0 ) were less likely to use annual preventive visits $(\mathrm{P}<0.01$ for each) during the study period (2007-2013). In contrast, after Medicare coverage expansion there was a significantly larger increase in the likelihood of using annual preventive visits 
among older seniors (OR: 1.5), males (OR: 1.1) and those who previously made frequent primary care visits (OR: 1.2 for 1 vs. 0 ; OR: 1.3 for 2 vs. 0 ; OR: 1.2 for $3+$ vs. 0 ; $\mathrm{P}<0.01$ for each).

Conclusion: Seniors who were older and frequent users of primary care had been underutilizing preventive care. Medicare's preventive service coverage expansion particularly benefited such seniors with needs for preventive care, but who may not have made separate preventive visits due to prior lack of coverage.

Keywords: Affordable Care Act, preventive care

A2-3:

Emergency Department Use in Alameda County, California, Before and After the Implementation of the Coverage Provisions of the Affordable Care Act

\author{
Alice Pressman, ${ }^{1}$ Anjali Franco, ${ }^{1}$ Sarah Robinson, ${ }^{1}$ Lisa \\ Dean-Gilley ${ }^{1}$
}

${ }^{1}$ Research, Development, and Dissemination, Sutter Health, Walnut Creek, CA

Background/Aims: Hospital emergency departments (EDs) across the United States struggle with overcrowding, a problem reported to be associated with decline in quality of care and patient satisfaction, decreased revenue and increased patient mortality. The 2010 Affordable Care Act (ACA), upon its full implementation on Jan. 1, 2014, extended health coverage to 32 million Americans. Initial studies have reported a rise in ED utilization post-ACA, but no formal analysis has been conducted in the Sutter Health system. We sought to characterize the change in ED use at two Sutter Health hospitals before and after ACA.

Methods: We reviewed demographic and hospital accounts data from California's Office of Statewide Health Planning and Development (OSHPD) as well as electronic health records for patients at two Sutter Health hospitals in Alameda County, California. We compared utilization and health care costs during comparable 6-month periods before and after the ACA expansion, and also examined trends over time from 2010 to 2014.

Results: From 2010 to 2013, encounters from OSHPD not resulting in a hospital admission were steady at the two hospitals; in 2014, they rose by $9 \%$. This was comprised of a $2 \%$ increase in commercial, $44 \%$ increase in Medicaid and $32 \%$ decrease in self-pay encounters. From records comparing the last 6 months of 2013 to the last 6 months of 2014 , total encounters rose by $11 \%$. While there were 3,143 fewer encounters by uninsured individuals, there were 7,478 more encounters by the insured (including individuals with Medicare, Medicaid and commercial). Charges increased by 20\% (commercial 29\%, Medicaid 59\%, Medicare 23\%, selfpay $-52 \%$ ) between the time periods.

Conclusion: ED use at the two hospitals increased after the implementation of the ACA's coverage expansions. The jump in aggregate ED visits came after at least three years of relatively stable ED use and was attributable to a shift in uninsured patients gaining Medicaid or commercial insurance coverage. Further research is required to determine the mechanism behind the increased utilization specific to insurance type and the possible implications for this extra burden on an already overcrowded ED.

Keywords: Affordable Care Act, emergency department overcrowding

A2-4:

Provider-Level Variation and Determinants of Outpatient Generic Drug Prescribing in a MixedPayer Health Care System

Robert Romanelli, ${ }^{1}$ Vani Nimbal, ${ }^{1}$ Jodi B. Segal ${ }^{2}$

${ }^{1}$ Palo Alto Medical Foundation Research Institute, Palo Alto Medical Foundation for Health Care, Research and Education, Palo Alto, CA; ${ }^{2}$ Department of Internal Medicine, Johns Hopkins School of Medicine, Baltimore, $M D$

Background/Aims: While $86 \%$ of all drugs in the United States are dispensed as generics, this rate is not uniform across all therapeutic classes and drug products. The role of the health care provider in influencing generic use is not well understood. We sought to quantify variation between providers and provider-specific determinants of generic prescribing in an outpatient setting.

Methods: This was a cross-sectional analysis of electronic health record data from a mixed-payer health care system in northern California in 2013. We focused on 12 therapeutic classes that had the potential for suboptimal generic utilization, including stimulants, antidepressants, thyroids, vasodilators, vascular 5HT1 agonists, antiplatelets, sympathomimetics, smooth-muscle relaxers, eyes/ears/nose/ throat drugs, estrogens, antigout agents and cardiac agents. Generic prescribing rates for each class were calculated, regardless of generic availability. For each class, we applied mixed-effect logit regression models with generic prescribed (yes/no) as the dependent variable and random effects (intercepts) for providers. Models included provider-level determinants: provider type (specialist vs. primary care), and continuous measures of average weekly prescription count and patient visits over the previous 3 months. We further adjusted for patient-level characteristics (age, sex, race/ ethnicity, insurance, comorbidities, prescription burden). For each model we calculated the intraclass correlation (ICC) as a measure of random provider-level variation and adjusted odds ratio (OR) for provider-level fixed-effect covariates.

Results: We identified 162,819 prescriptions in 2013 for 150,881 patients in the 12 therapeutic classes of interest. Generic prescribing rates across therapeutic classes ranged from $9.3 \%$ (sympathomimetics) to $95.1 \%$ (antidepressants). After controlling for provider and patient characteristics, significant between-provider variation in generic prescribing was observed; ICC ranged from $7.6 \%$ (vasodilators) to $72.9 \%$ (eyes/ears/nose/throat drugs). Specialists had lower odds of prescribing generic antidepressants than primary care 
providers (OR: 0.48), but higher odds of prescribing generic estrogens (OR: 1.55) and sympathomimetic agents (OR: 2.53). Across classes, a provider's prescription volume or patient volume had little or no effect on generic prescribing.

Conclusion: In this cross-sectional analysis of electronic health record data from a mixed-payer health system, we observed large heterogeneity across providers in outpatient generic prescribing for select therapeutic classes, even after accounting for patient characteristics. Health system initiatives may be warranted to better educate providers on generic prescribing.

Keywords: generic prescribing, provider variation

\section{PS1-07: \\ Classification and Mapping of Emergency Department Use in Three San Francisco Bay Area Hospitals}

\author{
Alice R. Pressman, ${ }^{1}$ Anjali Franco, ${ }^{1}$ Sarah Robinson, ${ }^{1}$ \\ Lisa Dean-Gilley, ${ }^{1}$ Maria Moreno ${ }^{1}$
}

${ }^{1}$ Research, Development, and Dissemination, Sutter Health, Walnut Creek, CA

Background/Aims: Preventable or nonurgent encounters to the emergency department (ED) disrupt patient flow and contribute to the burden of overcrowding. Furthermore, overcrowding is expected to increase as insurance coverage expands. We sought to create two categorization schema for nonurgent and/or preventable encounters to the ED and to visualize the resulting patient segments geographically in order to identify gaps in health care delivery services.

Methods: From electronic health records we extracted demographics, payer type and diagnoses for all encounters from June 2013 to May 2014 at three Sutter Health EDs in Alameda County, California. Based on primary diagnosis, we developed an algorithm to classify each encounter as urgent/nonurgent, and preventable/nonpreventable, collectively defined as inappropriate. We geocoded patient addresses and the addresses of all federally qualified health centers (FQHCs) in the area.

Results: During the study period, 69,595 individuals made 111,535 encounters to the study EDs; $36 \%(n=25,349)$ of individuals had at least one nonurgent encounter, whereas only $19 \% \quad(n=13,132)$ had one or more preventable encounters. Overall, approximately half of all patients had at least one type of inappropriate encounter. Demographics differed between those who used the ED inappropriately and those who did not. Nonurgent users were younger and those with preventable encounters were older compared to urgent and nonpreventable users, respectively. The distribution of minorities differed with more African-Americans having inappropriate encounters of both types. The proportion of uninsured did not vary by type of user $(n=57,290$ [82\%] of all patients had some type of insurance). We identified that $49 \%$ $(23,535$ of 48,156$)$ of inappropriate encounters were made by individuals who lived within one mile of an FQHC.

Conclusion: Using the methods described, we found that individuals who used the ED for nonurgent and/or preventable conditions tended to have some type of insurance coverage and were disproportionately African-American. By mapping the encounters and FQHCs, we were able to define catchment areas for each hospital and estimated that half of all inappropriate encounters could have been diverted to nearby, more suitable resources. Further identification and mapping of alternative health care and community resources will likely show that even more of these patients have alternative options available to them.

Keywords: emergency department overcrowding, inappropriate utilization

PS1-08:

Scaling Lean in Primary Care and Impacts on Organizational Performance

Dorothy Y. Hung, ${ }^{1}$ Michael I. Harrison, ${ }^{2}$ Meghan C. Martinez, ${ }^{1}$ Harold S. Luft ${ }^{1}$

${ }^{1}$ Palo Alto Medical Foundation Research Institute, Palo Alto Medical Foundation for Health Care, Research and Education, Palo Alto, CA; ${ }^{2}$ Center for Delivery, Organization, and Markets, Agency for Healthcare Research and Quality, Rockville, MD

Background/Aims: Despite increasing adoption of lean practices in health care, there are few rigorous studies of its impacts on delivery system performance over time, particularly in fee-for-service ambulatory settings. This study examines the impact of lean implementation on performance metrics, including workflow efficiencies, physician productivity, cost, quality and satisfaction in primary care departments of a large ambulatory care delivery system.

Methods: This was an observational study of phased lean implementation among 328 physicians in 46 primary care departments housed within 17 geographically distinct clinic locations. Performance metrics included: workflow efficiency, productivity, operating expenses, clinical quality and patient, physician and staff satisfaction. Interrupted time series analysis using generalized linear mixed models was used to examine lean impacts on organizational performance over time.

Results: Systemwide improvements were observed in workflow efficiencies, physician productivity and clinical quality metrics $(\mathrm{P}<0.05)$. Patient satisfaction with access to care, handling of personal issues and overall experience of care also increased, but decreased with respect to interactions with care providers $(\mathrm{P}<0.05)$. Departmental operating costs decreased, though this was not statistically significant. Finally, annual staff and physician satisfaction scores increased in key domains, ranging from employee engagement and connection to purpose to relationships with staff and physician time spent working.

Conclusion: Lean system redesigns resulted in improvements on a variety of metrics ranging from provider workflow efficiencies to satisfaction among patients and staff. This study contributes to a currently sparse literature on the impact of lean in primary care. In examining a comprehensive set of performance metrics, our findings suggest several improvements in areas targeted by lean redesigns.

Keywords: lean process improvement, delivery systems 
PS1-09:

\section{Patient Experiences in Selecting a Medicare Part D Prescription Drug Plan}

\author{
Cheryl D. Stults, ${ }^{1}$ Alison Baskin, ${ }^{2}$ Ming Tai-Seale,,${ }^{1} \mathrm{M}$. \\ Kate Bundorf ${ }^{3}$ \\ ${ }^{1}$ Palo Alto Medical Foundation Research Institute, Palo Alto \\ Medical Foundation for Health Care, Research and Education, \\ Palo Alto, CA; ${ }^{2}$ National Institutes of Health, Bethesda MD; \\ ${ }^{3}$ School of Medicine, Stanford University, Stanford, CA
}

Background/Aims: To obtain publicly subsidized prescription drug insurance, Medicare beneficiaries must enroll in one of the 28-39 private plans offered by their state. We sought to understand the experiences of individuals selecting a Part D plan, specifically what resources and which factors were of greatest importance to them.

Methods: Participants were patients of a large multispecialty group practice in northern California. Potential participants were recruited through flyers, several events for seniors and an article in the group practice's newsletter. Four focus groups were conducted from June through October 2014 until thematic saturation was reached. The 17 participants were all in a Medicare Part D plan, with ages ranging from 65 to 91 years (average of 74), primarily wealthy, retired and Caucasian. Focus groups were transcribed and qualitatively analyzed for main emergent themes.

Results: Most found the process of selecting a plan to be a "nightmare" to "extremely difficult" and "confusing" due to "too many options." Participants expressed that the characteristic of most importance to them in choosing a plan was the lowest cost, mainly through lower copays for medicines. Another important feature was the reputation of the company providing the plan to learn if "they make mistakes often," "handle claims satisfactorily," and "can you reach somebody if you need to." The main resources used in the decision-making process were other people (e.g. spouse, friend, family), books, brokers, AARP, the Health Insurance Counseling and Advocacy Program - a volunteer-supported program run by the California Department of Aging that provides unbiased information to help Medicare beneficiaries make the best choices for their individual health care needs - and the Internet, specifically the Medicare.gov website.

Conclusion: While low cost was a top priority for some, other participants reported that other characteristics like reputation of the company was so important to them that they were willing to pay a higher cost. More help from individuals and clearer information are necessary to assist Medicare beneficiaries in selecting a Medicare Part D prescription drug plan. Given that highly educated and affluent Medicare beneficiaries are finding the Part D plan selection process so difficult and complex, this poses a greater challenge for those with fewer resources.

Keywords: qualitative research, Medicare Part D
PS1-10:

Managing Multiple Conditions During an Acute Episode: Impacts on Costs and Clinical Quality

\author{
Su-Ying Liang, ${ }^{1}$ Laura J. Eaton, ${ }^{1}$ Harold S. Luft'
}

${ }^{1}$ Palo Alto Medical Foundation Research Institute, Palo Alto Medical Foundation for Health Care, Research and Education, Palo Alto, CA

Background/Aims: Managing multiple conditions during an acute visit often requires more comprehensive evaluation of concurrent conditions and is associated with higher pervisit cost. However, little is known about whether managing multiple conditions during a single visit affects overall costs or quality. This study examines this potential impact on the annual cost per patient and clinical quality.

Methods: Data were from electronic health records and claims of a large ambulatory group practice with mixed payment sources. We studied primary care physicians (PCPs) in 2010-2013 ( $\mathrm{n}=309)$ who had 1,099 PCP-years, the unit of analysis. Annual costs were expressed as the ratio of the observed costs to the expected costs adjusted for patient age, sex and clinical conditions. Costs included standardized fees for physicians, laboratory/imaging orders, specialist services and medications. For the measure of clinical quality, we combined various indicators pertinent to primary care practice (e.g. management of chronic conditions and preventive service) and created a composite average score (number of patients who met the target/ number of eligible patients for that measure). We analyzed the ratio of the observed score to the expected score adjusted for the composition of new (vs. established) patients in the PCP panel. The management of multiple conditions was identified through the diagnosis codes for treating "other" conditions during acute episodes. PCPs were classified into three tertiles based on the average number of other conditions addressed per episode across all acute episodes. We then tested whether costs and quality differed across the tertiles.

Results: The risk-adjusted costs were not significantly different across tertiles of "other conditions" (0.997 [ \pm $0.174], 1.005[ \pm 0.191]$ and $1.010[ \pm 0.269]$ for the low, middle and high groups, respectively). Managing other conditions during acute episodes was associated with higher quality: PCPs in the top tertile of other conditions addressed had higher quality scores than the middle (1.048 vs. 1.033 , $\mathrm{P}=0.06$ ) and low groups (1.048 vs. $1.030, \mathrm{P}=0.05)$.

Conclusion: Our preliminary findings suggest that managing multiple conditions during acute episodes may be associated with better care (higher quality but not higher costs per year). More refined analyses will be conducted to confirm the robustness of findings.

Keywords: cost, clinical quality 
PS1-11:

\section{The Impact of Narrow Provider Networks on Patterns} of Care

\section{Caroline C. Carlin, ${ }^{1}$ Bryan Dowd, ${ }^{2}$ Lucas Higuera $^{1}$}

${ }^{1}$ Medica Research Institute, Minnetonka, MN; ${ }^{2}$ Division of Health Policy and Management, University of Minnesota, Minneapolis, MN

Background/Aims: Our objective is to determine factors affecting choice of narrow provider network plans, and whether care covered by a narrow network preferred provider organization (PPO) plan differs from care provided in a plan with a broader PPO network. Narrow provider networks, when built around a single vertically integrated system, hold the potential for improvements in clinical care due to better information flow among providers, reduced duplication of tests and a more holistic knowledge of the patient.

Methods: The study sample includes employees enrolled through a private health insurance exchange who had coverage through one of the 93 employers offering network choice beginning in 2013. We follow enrollees through 2014 to identify the short-term impact of narrow network choice on patterns of health care delivery. Using claims data (2010-2014) from a regional health insurer offering a single-carrier private insurance exchange for employmentbased coverage, we predict enrollment in a narrow network plan option and use both instrumental variable methods and difference-in-differences methods to identify the association between narrow network enrollment and future patterns in health care delivery. Network options include a broad PPO network covering $95 \%$ of the primary care physicians in the region, and four narrow provider networks. Each narrow network is built around a single vertically integrated delivery system.

Results: Preliminary results indicate a statistically significant decline in both primary care and specialty care visits. The probability of accessing any care during the year was not impacted by narrow network enrollment, but overall utilization levels declined for those enrolled in a narrow network. There were no associations between narrow network enrollment and the risk of hospital-based care (emergency department visits, avoidable emergency department visits, inpatient admission) and no associations with the probability of preventive cancer screening (breast, cervical, colorectal).

Conclusion: It is encouraging to see reductions in specialty care visits without increases in emergency department or inpatient care or decreases in the probability of accessing any care in the year. While reductions in primary care visits indicate a need for caution, the pattern of reduced utilization may be a result of better provider knowledge of the patient due to care concentrated within a single care system.

Keywords: narrow provider networks, health care utilization
PS1-12:

\section{Cost-Minimization Analysis of Risk-Stratified Hematuria Evaluation}

Jing Hao, ${ }^{1}$ Tullika Garg,,${ }^{2}$ Susan R. Snyder, ${ }^{1}$ Amanda J. Young, ${ }^{1} \mathrm{H}$. Lester Kirchner ${ }^{1}$

${ }^{1}$ Center for Health Research, Geisinger Health System, Danville, PA; ${ }^{2}$ Geisinger Medical Center, Geisinger Health System, Danville, $P A$

Background/Aims: Up to $30 \%$ of adults have hematuria; however, $5 \%$ or fewer have a significant finding such as bladder or kidney cancer. Current guidelines recommend cystoscopy and imaging for all who meet criteria; however, the guidelines acknowledge evidence gaps resulting in substantial unnecessary testing with adverse financial implications for patients and society. Although riskstratified approaches have the potential to reduce testing, few are available and none have been rigorously evaluated. The objective of this study is to compare the direct health care costs of two hematuria evaluation approaches: American Urological Association (AUA) guidelinebased standard of care versus a theoretical risk-stratified algorithm.

Methods: We will apply decision-analysis modeling and simulation to conduct a cost-minimization analysis, a type of cost-effectiveness analysis that compares interventions of similar effectiveness, to compare the two hematuria evaluation approaches. Model inputs including assumptions and parameter values will be obtained from an established Geisinger Health System hematuria patient database. The database includes patient-level clinical information compiled from extensive chart review of 3,000 patients for whom hematuria evaluations were conducted from 2004 to 2014. Costs for services are based on Medicare reimbursement rates. Data from published literature and inputs from a clinical expert panel will supplement the model inputs.

Results: The results to be presented are a decisionanalysis model with assumptions, parameters and input values documented from the Geisinger hematuria database, literature review and clinical expert inputs. The analysis of deterministic decision model provides base-case clinical and economic results for the two approaches.

Conclusion: The finalized results of the cost-minimization analysis will enable threshold analysis to identify critical parameter values (e.g. sensitivity, specificity) for the performance of a theoretical risk-stratified approach to match the effectiveness of the current AUA guideline-based standard of care, and compare the direct health care costs of the two hematuria evaluation approaches from payer perspective. These results will provide a foundation of evidence and a model to evaluate risk-stratified approaches. Keywords: hematuria evaluation, cost-minimization analysis 
PS1-13:

\section{Pent-Up Demand After the Affordable Care Act}

Angela R. Fertig, ${ }^{1}$ Caroline S. Carlin, ${ }^{1}$ Scott B. Ode,${ }^{1}$ Sharon K. Long ${ }^{1}$

\section{${ }^{1}$ Medica Research Institute, Minnetonka, MN}

Background/Aims: Many state agencies, insurers and providers expected newly insured individuals under the Affordable Care Act (ACA) to inundate the health care market with high demand for health care. High health care utilization among newly insured individuals could indicate higher health risk and/or pent-up demand, defined as initial utilization caused by foregoing or delaying care while uninsured or underinsured. This study provides evidence regarding the relative health risk and pent-up demand for health care among newly insured adults in Medicaid as health reform rolls out.

Methods: We used claims data from a large health insurer to examine the first six months of Medicaid coverage for Minnesota adults 18-64 years old who were newly enrolled between January and March $2014(\mathrm{n}=4,252)$. The comparison group was nonelderly Minnesota adults with Medicaid coverage with this insurer in $2013(\mathrm{n}=21,556)$. We compared seven types of health care utilization over six months for new and ongoing enrollees: all office visits, new patient office visits, emergency department visits, inpatient stays, diagnostic procedures, all prescriptions filled, and new prescriptions filled. We estimated logit models of the probability of having each type of health care utilization adjusting for age, gender, race/ethnicity and enrollee neighborhood characteristics. We used the coefficient estimates from these models to predict the likelihood of utilizing services as new enrollees compared to ongoing enrollees.

Results: We found lower average predicted rates of health care utilization among new Medicaid enrollees compared to similar ongoing enrollees, with one exception - new enrollees were more likely to have a new patient visit. In addition, we found that utilization among new enrollees declined during the first six months of coverage in every category except prescriptions filled. Conclusion: Our analysis of the first six months of Minnesota's 2014 Medicaid expansion suggests both lower health care needs relative to similar ongoing enrollees and the presence of pent-up demand. This preliminary evidence suggests that both the longterm costs of covering the newly insured and the ongoing pressure on provider supply under the ACA may be lower than expected. Keywords: health reform, Medicaid

PS1-14:

The Effect of Hard-Stop Medicaid Payment Reform on Early Elective Deliveries

Heather M. Dahlen, ${ }^{1}$ Mac McCoullough, ${ }^{2}$ Angela R. Fertig, ${ }^{1}$ Bryan Dowd, ${ }^{3}$ William Riley ${ }^{2}$

${ }^{1}$ Medica Research Institute, Minnetonka, MN; ${ }^{2}$ School for the Science of Health Care Delivery, Phoenix, AZ; ${ }^{3}$ Health Services Research, University of Minnesota, Minneapolis, $M N$
Background/Aims: We assessed the impact of Texas's Medicaid payment reform for early elective delivery on clinical care practices and perinatal outcomes.

Methods: We used National Vital Statistics System data for the years 2009-2013, which contained birth certificate records from all U.S. states and the District of Columbia. Data were merged to the 2014-2015 Area Health Resource File, which provided information on county-level economic and primary care provider characteristics for the five years in our study. Our key outcomes included early elective deliveries (induced and cesarean sections combined as well as cesarean section only), gestational age, birth weight (total, low birth weight, very low birth weight, large for gestational age) and early nonelective deliveries. We employed a difference-in-differences strategy to isolate the effect of the hard-stop policy in Texas from unrelated underlying trends present in control states. Models were adjusted for characteristics of the delivery and mother, if a father was not present on the birth certificate, county-level economic and provider trends, state-specific time trends, state fixed effects and linear time trends.

Results: Relative to the control states, there were significant reductions in early elective inductions of labor among Medicaid deliveries in Texas, including reduced rates of cesarean births. Of Texas births paid by Medicaid, $11.2 \%$ were the result of early elective induction of labor prior to hard-stop legislation; after adjusting for changes in early inductions in comparison states, this share dropped by 1.1 percentage points $(\mathrm{P}<0.001)$. Birth outcomes also improved for babies covered by Medicaid in Texas, with relative increases in average gestational age and average birth weight.

Conclusion: Findings from this study suggest that the Medicaid hard-stop policy in Texas was effective in reducing the rate of early elective deliveries among the Medicaid population. As a result, babies covered by this policy reached an older gestational age and greater gestational weight. Since Texas had one of the highest rates of early elective delivery prior to enacting hard-stop, we would expect that states with similar baseline rates also would have relatively large reductions in early elective delivery rates should they pass hard-stop legislation.

Keywords: Medicaid payment reform, perinatal health

PS1-15:

Contraceptive Choice After the Affordable Care Act

Angela R. Fertig, ${ }^{1}$ Caroline S. Carlin, ${ }^{1}$ Bryan Dowd ${ }^{1,2}$

${ }^{1}$ Medica Research Institute, Minneapolis, MN; ${ }^{2}$ University of Minnesota, Minneapolis, MN

Background/Aims: The Affordable Care Act (ACA) mandated that, starting in late 2012, private health insurance plans that are not exempt or grandfathered were required to cover all contraceptive methods approved by the U.S. Food and Drug Administration as prescribed for women without a patient copayment. This policy has the potential to save 
billions in health care expenditures by reducing the number of unintended pregnancies, thereby reducing the number of deliveries and elective abortions. This study investigated the effect of the ACA-induced change in cost-sharing for contraceptive care on the rate of contraceptive use, the choice between long- and short-term contraceptive methods and the probability of elective abortion.

Methods: We used longitudinal health insurance claims data on female enrollees $18-45$ years old $(\mathrm{N}=29,990)$ in insurance plans obtained through 499 employer groups with at least 50 enrollees. Medical and pharmacy claims from 2008 through 2014 were extracted for the sample. Our control group was women covered by the employers who had not yet complied with the ACA-mandated cost sharing as of the end of 2014. We modeled their contraceptive choice using a multinomial probit regression with individual random effects to control for the woman's time-invariant unobserved characteristics. In a second regression, we modeled elective abortion using a binary probit regression with random effects.

Results: We found that when the copay for contraceptives fell to $\$ 0$ for those in compliant plans, contraceptive use rose substantially more than for those in noncompliant plans. Moreover, the mandate has increased the probability that a woman chose a long-term contraceptive method above and beyond the general increasing trend for these methods. We also observed a marginally significant decline in elective abortions.

Conclusion: These findings suggest that women are pricesensitive with regard to contraception choice, and thus the ACA mandate will likely significantly reduce the rate of unintended pregnancy. Because unwanted pregnancy is associated with poor birth outcomes, this policy also has the potential to reduce the fraction of high-cost births and children in poor health.

Keywords: health reform, maternal and child health

PS1-16:

\section{Patient Loyalty in a Mature IDS Market: Is Population Health Management Worth It?}

\section{Caroline S. Carlin ${ }^{1}$}

\section{${ }^{1}$ Medica Research Institute, Minneapolis, MN}

Background/Aims: The objective was to understand patient loyalty to providers over time in order to inform effective population health management. We studied patient careseeking patterns over a 6-year timeframe in Minnesota, an environment where care systems are motivated to practice population health management through shared-saving contracts with public and private payers.

Methods: A regional health plan provided data from their administrative files, matched to U.S. Census-based characteristics of the patient's neighborhood. Patients were retrospectively attributed to health care systems by the health plan based on patterns of primary care. Weibull duration and probit models were used to examine patterns of patient attribution to a care system and the continuity of patient affiliation with a care system. Clustering of errors within family unit was used to account for within-family correlation in unobserved characteristics that affect patient loyalty.

Results: We found significant patient loyalty, with past loyalty a very strong predictor of future relationship. Relationships were shorter when the patient's health status was complex and longer when their care system provided primary and multispecialty care.

Conclusion: Continuity of provider-patient relationships mean population health management can be beneficial to the care system that makes this investment, particularly for patients exhibiting prior continuity in care system choice. The results suggest that co-located primary and specialty services are important in maintaining primary care loyalty.

Keywords: organization and delivery of care, physician payment

PS1-17:

"Aging Out" of Dependent Coverage and the Effects on U.S. Labor Market and Health Insurance Choices

Heather M. Dahlen ${ }^{1}$

\section{${ }^{1}$ Medica Research Institute, Minnetonka, MN}

Background/Aims: We examined how labor market and health insurance outcomes were affected by the loss of dependent coverage eligibility under the Patient Protection and Affordable Care Act (ACA).

Methods: We used National Health Interview Survey data and regression discontinuity models to measure the percentage-point change in labor market and health insurance outcomes at age 26 . The sample was restricted to unmarried individuals 24 to 28 years old and to a period of time before the ACA's individual mandate (2011-2013). Models were run separately for men and women to determine if there were differences based on gender.

Results: Aging out of this provision increased employment among men, employer-sponsored health insurance offers for women and reports that health insurance coverage was worse than it was one year previously (overall and for young women). Uninsured rates did not increase at age 26, but there was an increase in the purchase of nongroup health coverage, indicating interest in remaining insured after age 26 .

Conclusion: Many young adults will turn to state and federal health insurance marketplaces for information about health coverage. Because young adults (age 18-29) regularly use social media sites, these sites could be used to advertise insurance to individuals reaching their 26th birthdays.

Keywords: Affordable Care Act, dependent coverage provision 
ECl Awardee:

Changes in Adolescent HPV Vaccination Following Incorporation Into California's Pay-for-Performance Program

Katharine A. Rendle, ${ }^{1}$ Caroline A. Thompson, ${ }^{1}$ Jennifer Ahern, ${ }^{2}$ Heidi M. Bauer, ${ }^{2}$ Sandra R. Wilson ${ }^{1}$

${ }^{1}$ Palo Alto Medical Foundation Research Institute, Palo Alto Medical Foundation for Health Care, Research and Education, Palo Alto, CA; ${ }^{2}$ Division of Epidemiology, School of Public Health, University of California, Berkeley, Berkeley, CA

Background/Aims: Pay-for-performance programs (P4P) are intended to improve care by aligning incentives with targeted outcomes; however, findings regarding their impact are mixed. Adolescent female human papillomavirus (HPV) vaccination was added to the measures reported by California's P4P program in 2012. Using electronic health record data from a mixed-payer, multispecialty health care organization in northern California, we examined on-time completion of the three-dose vaccination series by the recommended age of 13 before and after P4P implementation among 10,020 adolescent females.

Methods: We included all eligible female patients who turned 13 years old two years before (2010-2011 cohort) and two years after P4P implementation (2012-2013 cohort), excluding those patients with documented history of any adverse vaccine reaction. We used hierarchical logistic regression models to account for patient clustering within primary care providers. We estimated odds ratios (OR) associated with on-time vaccination completion with $95 \%$ confidence intervals (CI) based on robust estimation of standard errors, adjusting for patient race, insurance type and length of patient-provider relationship as well as provider experience (in years), gender, specialty and location.

Results: We found a positive association between on-time completion of HPV vaccination and P4P reporting of HPV vaccination. Patients who turned 13 after $\mathrm{P} 4 \mathrm{P}$ implementation had $27 \%$ higher odds of completing vaccination by age 13 than those who turned 13 before (OR: $1.27,95 \%$ CI: $1.12-1.45$ ), adjusting for all covariates. Compared with other groups, African-American and Asian Indian patients had significantly lower odds of on-time vaccination, and patients with public or HMO insurance had significantly higher odds of on-time vaccination. Despite increases, the proportion of patients who completed the series on time remained remarkably low $(12.5 \%$ [538/4,312] in the 2010-2011 cohort and $15.0 \%[855 / 5,708]$ in the 2012-2013 cohort).

Conclusion: Although further research is needed to establish any causal effects of P4P implementation on HPV vaccination uptake, these findings provide support for the continued use of incentive programs as a means to help improve health care quality and outcomes. On-time vaccination completion rates, however, remain suboptimal. Additional efforts, such as quality improvement initiated by this health care system in early 2014, may be needed to encourage parents and providers to vaccinate by age 13 .

Keywords: cancer prevention, performance incentives

\section{MENTAL HEALTH, ALCOHOL AND SUBSTANCE USE/ABUSE}

C4-1:

Gender Differences in Alcohol Screening, Brief Intervention, and Referral to Treatment in Primary Care

Cynthia Campbell, ${ }^{1}$ Constance Weisner, ${ }^{1}$ Felicia W. Chi, ${ }^{1}$ Thekla Ross, ${ }^{1}$ Stacy Sterling, ${ }^{1}$ Jennifer Mertens ${ }^{2}$

${ }^{1}$ Division of Research, Kaiser Permanente Northern California, Oakland, CA; ${ }^{2}$ Pediatric Developmental Disabilities Office, Kaiser Permanente Northern California, Oakland, CA

Background/Aims: Alcohol Screening, Brief Intervention, and Referral to Treatment (SBIRT) in adult primary care is efficacious in reducing hazardous drinking and is widely recommended. This study examines whether there are gender disparities in receipt of alcohol SBIRT among adult primary care patients by analyzing data from the ADVISe study, a clustered randomized controlled trial on SBIRT implementation by primary care physicians (PCP) and nonphysician providers (NPP) in a large, integrated health care delivery system.

Methods: This observational prospective cohort study examined electronic health record data on 639,593 adult patients who had a primary care visit at an ADVISe study clinic during Year 1 of the study. We conducted bivariate analyses to examine differences between males and females in demographic and clinical characteristics, screening rates and $\mathrm{BI} / \mathrm{RT}$ rates among those who screened positive. Within each intervention arm, multilevel logistic regression models were fitted to further assess the associations between patient gender and receipt of alcohol SBIRT while adjusting for other covariates at patient, physician and system levels and accounting for clustering within physician and within clinic correlation of patient outcomes.

Results: Findings from multilevel logistic regression models suggested that women were less likely to be screened in both intervention arms (odds ratios [95\% confidence intervals]: 0.78 [0.75-0.82] for the PCP arm and 0.82 [0.77-0.87] for the NPP arm). Among those who screened positive, women were less likely to receive $\mathrm{BI} / \mathrm{RT}$ in both intervention arms (odds ratios [95\% confidence intervals]: 0.60 [0.48-0.76] for the PCP arm and 0.62 [0.51-0.77] for the NPP arm). The presentation also will include findings of outcomes by gender and age.

Conclusion: Better understanding of the unique patient-, provider- and system-level factors that are associated with receipt of alcohol SBIRT and outcomes for males and females, and strategies to address such factors, are needed.

Keywords: alcohol screening, gender differences 
C4-2:

Alcohol Screening and Brief Intervention and Improved Health Outcomes in Hypertensive Patients: Results From an Implementation Study in Primary Care Setting

Felicia W. Chi, ${ }^{1}$ Constance Weisner, ${ }^{1}$ Stacy Sterling, ${ }^{1}$ Derek Satre, ${ }^{2}$ Thekla Ross ${ }^{1}$

${ }^{1}$ Division of Research, Kaiser Permanente Northern California, Oakland, CA; ${ }^{2}$ Department of Psychiatry, University of California, San Francisco, San Francisco, CA

Background/Aims: Many hypertensive patients drink at levels above recommended limits, which is associated with adverse blood pressure changes. Alcohol screening and brief intervention in adult primary care has been found efficacious in reducing hazardous drinking, yet little is known about the impacts on health outcomes. This study aims to evaluate the effect of alcohol screening and brief intervention on blood pressure control among adult hypertensive patients by analyzing data of the ADVISe study, a clustered randomized controlled trial on Screening, Brief Intervention, and Referral to Treatment (SBIRT) implementation by primary care physicians (PCP) and nonphysician providers in a large, integrated health care delivery system.

Methods: This observational prospective cohort study examined electronic health record data during 2-year followup on 4,086 hypertensive primary care patients $18+$ years old who screened positive for past-year risky drinking at baseline, of which 1,478 had a blood pressure measure at both the index screen and 18-month follow-up (36\%). We examined associations between documented brief intervention and change in blood pressure by fitting general linear models and addressed issues of potential selection and attrition bias by using inverse probability weighting.

Results: Findings suggested a positive association between receiving brief intervention for positive hazardous drinking screen and better blood pressure control at 18 months for those in the PCP arm, for those with less severe drinking level at index screen and for those with poor blood pressure control at the index screen. For hypertensive patients with systolic/diastolic blood pressure at $140+/ 90+\mathrm{mmHg}$ who reported 1-7 hazardous drinking days in the past year, the adjusted mean decline of systolic blood pressure was 37.9 $\mathrm{mmHg}$ for those who received brief intervention compared to $17.2 \mathrm{mmHg}$ among those who did not receive brief intervention $(\mathrm{P}=0.0003)$. For those in the $\mathrm{PCP}$ arm who had less severe drinking level at index screen, those who received brief intervention had 10 times higher odds of having hypertension under control than those who did not. Conclusion: Preliminary results suggest that alcohol screening and brief intervention may be beneficial for adult primary care patients with hypertension.

Keywords: alcohol screening and brief intervention, hypertension
C4-3:

Association of OPRM1 Polymorphisms With DSM5-Defined Prescription Opioid Use Disorder Among Chronic Pain Patients on Opioid Therapy for Noncancer Pain

Joseph A. Boscarino, ${ }^{1}$ Stuart N. Hoffman, ${ }^{1}$ John J. Han, ${ }^{2}$ $\mathrm{Xin} \mathrm{Chu}^{3}$

${ }^{1}$ Center for Health Research, Geisinger Health System, Danville, PA; '2Department of Pain Medicine, Geisinger Health System, Danville, PA; ${ }^{3}$ Weis Center for Research, Geisinger Health System, Danville, PA

Background/Aims: The OPRM1 $118 \mathrm{~A}>\mathrm{G}$ polymorphism (rs1799971) has been associated with susceptibility to drug misuse. Previously we assessed the association between single nucleotide polymorphisms (SNPs) located in OPRM1 genetic locus, including rs1799971 and rs6923231, and susceptibility to substance misuse among outpatients receiving long-term opioid therapy.

Methods: We identified outpatients receiving opioid therapy for noncancer pain within a large health care system using electronic health records. Patients included those $18+$ years old who received opioids for $4+$ months in the past 12 months. We completed diagnostic interviews with 705 subjects to identify substance use disorders including opioid use disorder and history of illicit drug use. Genotyping was performed on an Applied Biosystems ${ }^{\mathrm{TM}} 7500$ real-time polymerase chain reaction platform, using TaqMan ${ }^{\text {TM }}$ kits.

Results: Mean age of patients was 55 years (SD: 13.4), and $60.9 \%$ (95\% confidence interval [CI]: 55.9-65.7) were female. Based on Diagnostic and Statistical Manual of Mental Disorders, Fourth Edition (DSM-IV) criteria, the lifetime prevalence of opioid dependence was 35.6\% (95\% CI: 32.1-39.1). Lifetime illicit drug use was $12.8 \%$ (95\% CI: $10.3-15.2)$ for cocaine and $14.9 \%$ (95\% CI: $12.3-17.5)$ for amphetamines/methamphetamines. Using ordinal logistic regression that controlled for age, gender, education and marital status, lifetime opioid use disorder by DSM- 5 criteria (classified: none, mild, moderate, severe) was associated with the carriers of the G allele (coded 0,1 or 2) at SNP rs6923231 (odds ratio: 1.48, $\mathrm{P}=0.002$ ), but the OPRM1 $118 \mathrm{~A}>\mathrm{G}$ polymorphism (rs1799971) was not associated $(\mathrm{P}=0.544)$. Multivariate linear regressions suggested the number of days the patient used cocaine (range: $0-100$ days) was significantly associated with SNP marker rs6923231 ( $\beta=0.72, P=0.0000565$ ), as was the number of days the patient used amphetamines/methamphetamines (range: 0-500 days; $\beta=0.09, P=0.046$ ). When we assessed opioid disorders based on DSM-IV criteria, neither SNP marker rs6923231 nor rs1799971 was associated.

Conclusion: We detected genetic liability for addiction based on the DSM- 5 criteria at the OPRM1 genetic locus for SNP rs6923231, a previously unreported polymorphism for drug misuse. Since the SNPs for this study were identified using a tagging algorithm that was correlated with addiction phenotypes, SNP rs6923231 is likely a valid marker for addiction liability. To our knowledge, this is the first study to 
report genetic associations for OPRM1 variants based on the DSM-5 criteria for prescription opioid use disorder.

Keywords: prescription opioid use disorder, chronic pain patients

\section{C4-4: \\ Implementing Collaborative Care Management for Depression, Diabetes, and Cardiovascular Disease Across Eight Health Care Systems}

Arne Beck, ${ }^{1}$ Jennifer Boggs, ${ }^{1}$ Angelika Clarke, ${ }^{1}$ Karen Coleman, ${ }^{2}$ Claire Neely, ${ }^{3}$ Tani Hemmila, ${ }^{3}$ Kris Ohnsong, ${ }^{4}$ Leif I. Solberg ${ }^{4}$

${ }^{1}$ Institute for Health Research, Kaiser Permanente Colorado, Denver, CO; ${ }^{2}$ Department of Research and Evaluation, Kaiser Permanente Southern California, Pasadena, CA; ${ }^{3}$ Institute for Clinical Systems Improvement, Bloomington, $\mathrm{MN}$; ${ }^{4}$ HealthPartners Institute for Education and Research, Minneapolis, MN

Background/Aims: Collaborative care management is effective for improving care of patients with depression and chronic medical conditions. The Care of Mental, Physical, and Substance use Syndromes (COMPASS) project implemented evidence-based core collaborative care management components needed to achieve improvement in patients with both depression (PHQ-9 > 10) and uncontrolled diabetes (hemoglobin A1c > 8.0) or cardiovascular disease (systolic blood pressure $>145$ or low-density lipoprotein $>100$ ). Components included measurement-based care, use of a care manager and registry, systematic case reviews, treatment intensification when indicated, relapse/exacerbation prevention and data evaluation for quality improvement. Supported by an award from the Centers for Medicare \& Medicaid Services, COMPASS was implemented through a collaborative of eight health systems and over 190 clinics in eight states. We used qualitative data to describe variation in the implementation of core COMPASS components across sites.

Methods: Implementation data across the eight COMPASS sites were obtained from annual site visit reports prepared by the Institute for Clinical Systems Improvement and qualitatively analyzed using Atlas.ti software, yielding emergent themes related to implementation facilitators and impediments. The Consolidated Framework for Implementation Research (CFIR) was used to organize qualitative data from site visits and as a conceptual framework for understanding implementation variation across COMPASS sites. CFIR includes six broad categories of implementation: intervention characteristics, outer setting, inner setting, implementation climate, characteristics of individuals, and process. Each broad category contains three to eight implementation subcategories.

Results: Four overarching themes were identified from site visit reports: 1) between-site differences; 2) challenges to implementation; 3) COMPASS learning and impact on health system; and 4) staff (characteristics, turnover issues, training, background). Nine additional subthemes were identified that were mapped to CFIR implementation categories and that demonstrated considerable cross-site variation: 1) primary care physician engagement; 2) prior experiences with care coordination; 3) length of patient enrollment in COMPASS model; 4) team dynamics; 5) care manager characteristics; 6) quality improvement reports; 7) registry use; 8) patient social needs; and 9) organizational environment where COMPASS was implemented.

Conclusion: Understanding the sources of variation in largescale collaborative care management implementation is critical to increase the odds for further successful dissemination of similar models.

Keywords: collaborative care, implementation research

PS1-34:

\section{Antidepressant Adherence Across Diverse Populations and Health Care Settings}

Rebecca C. Rossom, ${ }^{1}$ Susan Shortreed, ${ }^{2}$ Karen J. Coleman, ${ }^{3}$ Arne Beck, ${ }^{4}$ Beth Waitzfelder, ${ }^{5}$ Christine Stewart, ${ }^{2}$ Brian K. Ahmedani, ${ }^{6}$ John Zeber, ${ }^{7}$ Greg Simon ${ }^{2}$

${ }^{1}$ HealthPartners Institute for Education and Research, Minneapolis, MN; ${ }^{2}$ Group Health Research Institute, Seattle, WA; ${ }^{3}$ Department of Research \& Evaluation, Kaiser Permanente Southern California, Los Angeles, CA; ${ }^{4}$ Institute for Health Research, Kaiser Permanente Colorado, Denver, CO; 5 Institute for Health Research, Kaiser Permanente Hawaii, Honolulu, HI; ${ }^{6}$ Center for Health Policy and Health Services Research, Henry Ford Health System, Detroit, MI; ${ }^{7}$ Center for Applied Health Research, Baylor Scott \& White Health, Temple, TX

Background/Aims: Much of the work in antidepressant adherence relies on self-report and small sample sizes with limited racial/ethnic representation. Our study aimed to determine factors associated with poor early adherence to antidepressants in a large diverse sample of patients using pharmacy refill data.

Methods: Electronic medical record data for patients 18 and older with depression who filled a new outpatient antidepressant prescription between Jan. 1, 2010, and Dec. 31, 2012, in one of six Mental Health Research Network health care systems were obtained. Self-reported race/ethnicity and pharmacy fill data were obtained from electronic medical records. Patients were considered to have early adherence if they had a second antidepressant fill within 180 days of the first.

Results: 177,469 adult patients had 184,967 new episodes of depression associated with a filled antidepressant prescription. Patients refilled their antidepressants within 180 days of the first dispensing in $71 \%$ of treatment episodes. Race/ethnicity was a strong predictor of early adherence, with patients who self-identified as Asian, non-Hispanic black, Hispanic or Native Hawaiian/Pacific Islander significantly less likely to refill their antidepressant prescriptions than were non-Hispanic whites or Native Americans/Alaskan Natives. Other apparent predictors of early adherence, including neighborhood income and education, gender and prior mental 
health hospitalizations, were no longer significant after adjusting for race/ethnicity.

Conclusion: Race/ethnicity was a robust predictor of antidepressant adherence, with racial/ethnic minority groups other than Native Americans/Alaskan Natives less likely to be adherent. Patients from these racial and ethnic minority groups may have the greatest potential benefit from targeted interventions to improve early antidepressant adherence.

Keywords: depression, adherence

PS1-35:

Early Identification of Autism Spectrum Disorders Using Patterns of Service Use Prior to Diagnosis

Frances L. Lynch, ${ }^{1}$ Lisa A. Croen, ${ }^{2}$ Ashli Owen-Smith, ${ }^{3}$ Robert L. Davis, ${ }^{4}$ Ginger C. Hanson, ${ }^{1}$ Phillip M. Crawford, ${ }^{1}$ Kristal C. Ruse,${ }^{1}$ Yinge X. Qian ${ }^{2}$

${ }^{1}$ Center for Health Research, Kaiser Permanente Northwest, Portland, OR; '2Division of Research, Kaiser Permanente Northern California, Oakland, CA; ${ }^{3}$ Center for Health Research, Kaiser Permanente Southeast, Atlanta, GA; ${ }^{4}$ Health Science Center, University of Tennessee, Memphis, TN

Background/Aims: Early intervention services in the first few years of life significantly improve outcomes for children with autism spectrum disorders (ASD). Yet there are often delays in the identification and diagnosis of ASD. If health systems were better able to identify children at high risk for ASD and bring them in for comprehensive evaluation earlier, more children could be able benefit from early intervention.

Methods: This study used data from three Kaiser Permanente sites (Northern California, Georgia, Northwest). All children born between Jan. 1, 2000, and Dec. 31, 2009, were included in the study population. All data stored in the electronic medical records up to June 2012 were collected. Children with an ASD diagnosis from an ASD specialist or two or more ASD diagnoses from nonspecialists were considered to have a valid ASD diagnosis. Controls were matched to cases at a 10:1 ratio and were matched on age, birth month and membership in the 12 months prior to the case's first ASD diagnosis. We used two-part regression models and cluster analysis to examine whether children diagnosed with ASD have different patterns of service use prior to their first ASD diagnosis than children who do not go on to receive an ASD diagnosis.

Results: Standard two-part regression models and cluster analyses identified differential patterns of service use prior to diagnosis for children with ASD compared to children without ASD. Regression models indicated that children with ASD have much greater use of specialty care (e.g. mental health services, neurology) prior to first ASD diagnosis. Cluster analyses provided a more nuanced picture. For example, early-onset ASD cases had a distinctive pattern separate from those who received a diagnosis at an older age.

Conclusion: Using data on patterns of service use prior to the initial ASD diagnosis, it may be possible to identify children who are likely on a trajectory to get an ASD diagnosis. By developing and applying algorithms that can identify children most at risk in the first few years of life, comprehensive evaluation could be done at an earlier age, improving the outcomes for these children.

Keywords: autism spectrum disorders, diagnosis

PS1-36:

Mobile Assessment of Depression Treatment Response: Recruitment Challenges and Possible Solutions

Ashli A. Owen-Smith, ${ }^{1}$ Michelle Panneton, ${ }^{2}$ Patricia Arean, ${ }^{3}$ Joaquin A. Anguera, ${ }^{4}$ Shelby Reetz, ${ }^{5}$ Greg N. Clarke $^{5}$

${ }^{1}$ Department of Health Management and Policy, Georgia State University, Atlanta, GA; ${ }^{2}$ Center for Clinical and Outcomes Research, Kaiser Permanente Southeast, Atlanta, GA; ${ }^{3}$ Department of Psychiatry and Behavioral Sciences, University of Washington, Seattle, WA; ${ }^{4}$ Department of Psychiatry, Department of Neurology, University of California, San Francisco, San Francisco, CA; ${ }^{5}$ Center for Health Research, Kaiser Permanente Northwest, Portland, OR

Background/Aims: Effective treatments to depression exist, but treatment response is variable. Persons likely to respond to antidepressants often show early change in emotional processing and attentional bias. Methods to assess these constructs passively and at low burden/cost are now available via smartphone apps, with potential for broad dissemination. Methods: This Mental Health Research Network-supported study is testing the feasibility, acceptability and utility of assessing behavior and neuropsychological performance for prediction of depression treatment response using smartphone behavioral apps. Kaiser Permanente Northwest $(\mathrm{N}=75)$ and Georgia members $(\mathrm{N}=75)$ starting new antidepressant treatment are recruited via secure online messaging through their www.kp.org personal health record. Participants complete assessments at baseline, 4, 8 and 12 weeks. App data is shared with their provider.

Results: We have experienced several challenges related to study enrollment. First, read rates of the recruitment message are at $\sim 60 \%$. Second, of those who read the message, only $12 \%$ visit the study website. Third, among those who visit the study website, only $\sim 3 \%$ consent and enroll in the study. Finally, among enrolled participants, approximately 33\% successfully download and use the apps. We have implemented several strategies aimed at increasing enrollment: 1) revising recruitment messaging to highlight the unique features of the apps and emphasize that results may help improve their treatment, 2) sending additional recruitment messages via email/text, 3) initiating recruitment calls using Kaiser Permanente's automated telephone system, 4) providing follow-up phone calls to offer technical assistance for those experiencing difficulties with downloading/using the apps, and 5) introducing tiered incentives to motivate completion of each phase of the study. These efforts appear to be 
improving our recruitment efforts. Additional interviews will be conducted with participants, and thus additional data about barriers to/facilitators of recruitment and retention will be available prior to the conference.

Conclusion: The use of mobile technologies to predict depression treatment response may have significant implications for effective and timely depression care. Understanding the barriers/facilitators of successful enrollment and retention is critical to the implementation of this intervention. Lessons learned from the present study may aid others in similar efforts and in different settings.

Keywords: depression, mobile assessments

\section{PS1-37:}

\section{Making Health Changes: Following Preventive Service Guidelines and Changing Behavior Among Individuals With Serious Mental IIInesses}

\section{Scott P. Stumbo, ${ }^{1}$ Bobbi Jo Yarborough, ${ }^{1}$ Micah T. Yarborough, ${ }^{1}$ Nancy Perrin, ${ }^{1}$ Carla A. Green ${ }^{1}$}

\section{${ }^{1}$ Center for Health Research, Kaiser Permanente Northwest, Portland, OR}

Background/Aims: People with serious mental illnesses generally experience greater morbidity and earlier mortality than individuals without mental health diagnoses. Understanding factors that influence preventive service use and assist people in making healthy behavior changes may reduce health disparities in this population.

Methods: This mixed-methods study, conducted in an integrated health system, federally qualified health centers and safety net clinics, derived diagnoses and needed preventive services from the electronic medical record to identify a sample $(\mathrm{N}=142)$ representing a range of prevention needs. Individuals from four diagnostic groups (schizophrenia spectrum disorders $[n=41]$, bipolar disorders/affective psychoses $[n=48]$, major depressive disorders $[n=33]$, anxiety disorders $[n=20]$ ) completed questionnaires and semi-structured interviews. Survey data were analyzed using SPSS; interviews were transcribed verbatim and coded using Atlas.ti.

Results: Nearly $50 \%$ of participants reported they would be "extremely likely" to make changes their doctor recommended on completing lab work, mammogram screenings, getting a flu shot and completing colon cancer screening. Only 20\% reported they were extremely likely to improve nutrition or increase exercise, even with doctor recommendations. Among smokers, 28\% reported they were "not at all likely" to follow doctor recommendations to stop or cut back. Reported likelihood did not differ by gender, diagnostic group or study site. While $46 \%$ reported they were "extremely" or "very confident" in following through with recommended health changes, $78 \%$ reported they could use at least "a little more support" with making recommended changes. Narrative analyses revealed three themes related to making changes: 1) making and sustaining health-related behavioral changes (e.g. losing weight/stopping smoking) were difficult compared with completing preventive services (e.g. doing lab work), 2) additional targeted support was needed to make and sustain complex changes (e.g. weight loss), and 3) lack of knowledge about reasons for preventive care inhibits completion of some services (e.g. colorectal screening).

Conclusion: Among people with serious mental illnesses, likelihood of following doctor recommendations is relatively low for important behavioral changes such as stopping smoking or increasing exercise. Additional support is needed to encourage completion of some difficult or invasive preventive services and for making and sustaining significant changes in health-related behaviors.

Keywords: behavior change, preventive service use

PS1-38:

Provider Perspectives on Behavioral Health Care for Adolescents: Barriers and Suggestions for Improvement

\section{Martina Li, ${ }^{1}$ Jinnan Li, ${ }^{1}$ Amy Meehan, ${ }^{1}$ Judith Chuang, ${ }^{1}$ Ellis C. Dillon, ${ }^{1}$ Ming Tai-Seale ${ }^{1}$}

${ }^{1}$ Palo Alto Medical Foundation Research Institute, Palo Alto Medical Foundation for Health Care, Research and Education, Mountain View, CA

Background/Aims: Onset of behavioral health needs commonly occurs at adolescence, and best practices call for early detection and coordinated care. Practices for assessing and treating these needs are not standardized and may vary widely. This research explores current provider practices and perspectives as a first step towards designing better strategies for adolescent behavioral health care.

Methods: Primary care providers (PCPs) within a multispecialty delivery system were surveyed and interviewed about assessment and treatment of adolescent behavioral health needs, barriers to appropriate care and suggestions for system improvement.

Results: Of 123 PCPs who completed the survey, 103 $(83.7 \%)$ felt their teen patients were willing to discuss behavioral health, $94(76.4 \%)$ felt their teen patients would want them to treat their behavioral health problems and 95 (76.9\%) were confident in their ability to identify behavioral health needs. However, 61 (49.6\%) PCPs were unaware of available resources, $63(51.2 \%)$ lacked confidence in making effective referrals, $65(52.8 \%)$ reported limited time was a barrier, and $72(58.5 \%)$ found behavioral health evaluations overburdening. Analyses of interviews with 21 PCPs revealed a lack of confidence among PCPs in assessing and treating less commonly seen adolescent behavioral health needs such as bipolar disorder, schizophrenia, substance abuse and suicidality. Other barriers to adolescent behavioral health care included: lack of time during appointments, lack of standardized referral process, and difficulties in accessing specialty behavioral health providers. PCPs desired improved care coordination, accessible behavioral health specialists within the delivery system for better continuity and coordination of care in contrast to outside specialists, 
and additional resources to help teen patients and families navigate the system.

Conclusion: While confident about identifying behavioral health needs among their teen patients, many PCPs report multiple challenges to providing necessary and appropriate care to treat their patients. Further research and quality improvement efforts are needed to improve existing resources and provide adequate support for providers to ensure adolescent behavioral health needs are expertly addressed.

Keywords: adolescent behavioral health, barriers to care

\section{PS1-39:}

\section{Adolescent Psychiatric Comorbidities Prospectively Predict Greater Asthma-Related Emergency Health Care Visits}

\section{Megan Flynn, ${ }^{1}$ Scott B. Ode ${ }^{1}$ \\ ${ }^{1}$ Medica Research Institute, Minneapolis, MN}

Background/Aims: Mismanagement of asthma, a prevalent chronic illness in which breathing airways are narrowed, can be life-threatening. Although psychiatric diagnoses are known to hinder self-care, less is known about the individual and combined impact of three relatively common psychiatric diagnoses (i.e. depression, anxiety, attention deficit disorder) on the management of asthma during adolescence, a developmental stage at which risk for psychiatric diagnoses is heightened and enduring health habits are established. The goal of this study was to examine whether the co-occurrence of adolescent psychiatric diagnoses (i.e. comorbidities) interferes with the management of asthma, specifically by contributing to more asthma-related emergency health care visits across three years.

Methods: Health insurance claims records were extracted for a sample of 1,158 adolescents (age 10-19 years; 47\% female) diagnosed with asthma who had continuous enrollment across three years in a regional health plan. Diagnostic grouping software identified diagnoses of depression, anxiety and attention deficit disorder as well as their comorbidities. Place of service codes captured asthma-related emergency department and urgent care visits. General linear modeling was used to examine whether psychiatric comorbidities predicted the average number of asthma-related emergency health care visits across three years. Specifically, for the focal independent variable, adolescents were categorized according to the number of psychiatric diagnoses present; for the focal dependent variable, the number of emergency department and urgent care visits were averaged for each month of enrollment across the three study years.

Results: In the comprehensive model, which controlled for potential covariates (i.e. gender, age, SES, months of enrollment), the number of psychiatric comorbidities significantly positively predicted the average number of emergency visits across three years $\left(\chi^{2}=8.41, \mathrm{P}<0.01\right)$.

Conclusion: Adolescents with greater psychiatric comorbidities experienced significantly more asthma-related emergency health care visits over three years. Findings highlight the need to consider the complex interplay between psychological health and chronic illness management during adolescence.

Keywords: adolescent mental health, asthma

PS1-40:

Adolescent Psychiatric Comorbidities Contribute to Diabetes-Related Emergency Health Care Visits

\section{Megan Flynn, ${ }^{1}$ Scott B. Ode ${ }^{1}$ \\ ${ }^{1}$ Medica Research Institute, Minneapolis, MN}

Background/Aims: Type 1 diabetes (T1D) is a chronic illness involving failure to produce insulin, and requires a demanding management regime to protect against serious ailments including stroke and cardiovascular disease. Although psychiatric diagnoses hinder selfcare, less is known about the impact of three relatively common psychiatric diagnoses (i.e. depression, anxiety, attention deficit disorder) on the management of T1D during adolescence, a developmental stage at which risk for psychiatric diagnoses is heightened, metabolic control declines and enduring health habits are established. The goal of this study was to examine whether the co-occurrence (i.e. comorbidity) of psychiatric diagnoses interferes with the management of T1D, specifically by contributing to a higher number of T1D-related emergency health care visits across three years in adolescence.

Methods: Health insurance claims records were extracted for a sample of 145 adolescents (age 10-19 years; $52 \%$ female) diagnosed with T1D who had continuous enrollment in a regional health plan across the three study years. Diagnostic grouping software identified diagnoses of depression, anxiety and attention deficit disorder, and their comorbidities. Place of service codes captured T1D-related emergency department and urgent care visits.

Results: A random effect logistic regression was used to examine whether psychiatric comorbidities increased the probability of T1D-related emergency health care visits within a study year. Potential covariates (i.e. gender, age, insurance policy type, months of enrollment) were included; the no psychiatric diagnosis group was used for comparison. Although single psychiatric diagnoses did not increase the probability of an emergency health care visit $(\mathrm{P}>0.50)$, psychiatric comorbidities significantly positively predicted emergency health care visits $(\mathrm{P}<0.001)$. Followup analysis revealed a marginal effect of $+22 \%$, indicating that the presence of psychiatric comorbidities substantially increased the likelihood of an emergency health care visit.

Conclusion: Results supported the hypothesis that psychiatric diagnoses have a synergistic effect on adolescents' ability to successfully manage T1D. These findings highlight the need to consider the complex interplay between psychological health and chronic illness management across adolescence. Keywords: adolescent mental health, diabetes 


\section{PATIENT, PROVIDER AND HEALTH SYSTEM ENGAGEMENT}

\section{C5-1: \\ Pathway to Measuring Patient and Family Engagement: Prioritizing Engagement Behaviors in the Hospital Setting}

Emily A. Elstad, ${ }^{1}$ Mangrum Rikki, ${ }^{1}$ Pam K. Dardess,,${ }^{1}$ Eric Johnson ${ }^{2}$

${ }^{1}$ American Institutes for Research, Chapel Hill, NC; ${ }^{2}$ American Institutes for Research, Washington, DC

Background/Aims: Growing evidence suggests that patient and family engagement (PFE) can help achieve the "Triple Aim" of improving population health and patient experiences of care while reducing costs. One critical gap as we work toward these goals is the lack of a clear approach to PFE measurement to assess associated outcomes and gauge health care performance. In this project, we draw on an existing framework to define behaviors that constitute PFE in the hospital setting and identify a subset of these behaviors that are of high priority for measure development and implementation. Methods: We identified engagement behaviors for hospital leadership, clinicians and patients/families through: a literature scan of publications and existing measures; key informant interviews; and input from an eight-member advisory group that included patients and families, clinicians, measurement experts and hospitals. To prioritize engagement behaviors, we categorized these behaviors into thematic domains, surveyed our advisory group and other experts in PFE and synthesized feedback to select a set of high-priority behaviors for PFE measurement that address multiple domains, are feasible to measure and are important for driving change or increasing accountability.

Results: Eight domains of engagement in the hospital setting were prioritized: access to medical record, creating opportunities for engagement, communication, discharge planning, family presence, goal-setting, shared care planning, and transparency. These domains include specific highpriority engagement behaviors at the hospital, clinician and patient levels. For example, priority behaviors in the "access to medical record domain" include: hospitals make medical records easily accessible and involve patients and families in designing medical record access mechanisms and policies; clinicians record patient medical information in plain language (free of jargon and abbreviations); and patients access and use their medical record.

Conclusion: PFE measurement is at a nascent stage. To our knowledge, this is the first work to systematically identify behaviors that constitute PFE in the hospital setting and prioritize those that are feasible and important to measure. The resulting matrix of high-priority measurement behaviors mapped against domains and levels of PFE provides a comprehensive approach for measuring PFE in the hospital setting, including the selection and development of measures. Keywords: patient and family engagement, hospital quality improvement
C5-2:

An Examination of Mentoring and Training in the Health Care Systems Research Network

\author{
Sharon L. Larson, ${ }^{1}$ Marc Williams, ${ }^{1}$ Ella Thompson, ${ }^{2}$ \\ Amber Eruchalu, ${ }^{3}$ Lela McFarland ${ }^{4}$ \\ ${ }^{1}$ Center for Health Research, Geisinger Health System, \\ Danville, PA; ${ }^{2}$ Group Health Research Institute, Seattle, \\ WA; ${ }^{3}$ Catholic Health Initiatives, Englewood, CO; \\ ${ }^{4}$ Catholic Health Initiatives, Baltimore, MD
}

Background/Aims: The Health Care Systems Research Network (HCSRN) commissioned a survey to characterize mentoring and training within member organizations and to identify gaps in mentoring and training at the member organization and network levels. In an era of increasing focus on team science and multidisciplinary teams, it is important for organizations such as HCSRN to understand the capacity of its member organizations to train and develop a new generation of researchers. The goals of this study were to: describe mentoring within organizations, identify gaps as well as strong programs of mentoring, and identify other meetings that our researchers attend to understand the education and training of interest.

Methods: A 14-item electronic survey was distributed to HCSRN board members for distribution to all researchers in each organization. This represents a descriptive analysis of responses.

Results: A total of 157 researcher/scientists and executives from 19 organizations responded to the survey. Responses by career level included 12 institutional leaders, 35 junior faculty, 33 midlevel faculty and 60 senior faculty. About half of all respondents indicated that there is a formal mentoring program in their organization. The most frequently reported characteristics of mentoring programs included: 1) development of joint applications by junior and senior researchers (49\%), 2) advocacy by mentor (40\%), 3) methods training (39\%), and 4) formal mentoring meetings $(39 \%)$. Further analysis of characteristics of mentoring will be presented.

Conclusion: There are a number of opportunities to shape a network approach to mentorship by capitalizing on existing approaches taken by individual organizations within the network. There were several suggestions for the development of mentoring networks and mentorship training and to provide senior researchers with tools and resources to mentor their protégés. Additionally, the network may offer didactic training in grantsmanship, project management and portfolio development for investigators at the junior, midcareer and senior levels of research within the purview of training that HCSRN can provide to its membership. Finally, many respondents requested training in specific methodologies that may warrant the development of a webinar series for all career levels.

Keywords: mentor, scientific education 
C5-3:

\section{A Clinical Decision Support System Promotes Shared Decision-Making and Cardiovascular Risk Factor Management}

JoAnn M. Sperl-Hillen, ${ }^{1}$ A.L. Crain, ${ }^{1}$ Heidi L. Ekstrom, ${ }^{1}$ Karen L. Margolis, ${ }^{1}$ Patrick J. O'Connor ${ }^{1}$

${ }^{1}$ HealthPartners Institute for Education and Research, Minneapolis, MN

Background/Aims: Cardiovascular (CV) Wizard is a web-based electronic health record-integrated point-ofcare clinical decision support (CDS) system that presents personalized CV risk information to providers and patients in both a low-numeracy visual format and a high-numeracy quantitative format. We report primary care provider perspectives on how this CDS system affected shared decision-making and $\mathrm{CV}$ risk factor management.

Methods: Twenty clinics were randomized to either usual care or use of the CDS system with diabetes, heart disease or high-reversible $\mathrm{CV}$ risk adults. The CDS system targeted $20 \%$ of office visits and was used at $70-80 \%$ of targeted visits over a 2-year period. Consented providers $(\mathrm{N}=102)$ were surveyed at baseline and 18 months after implementation. Corrected survey response rates were $90 \%$ at baseline and $82 \%$ at follow-up. Generalized linear mixed models were used to compare usual care and CDS responses to common questions at baseline and follow-up, and CDS users were queried on their perceptions of the CDS system at followup only.

Results: Compared to usual care providers, those in the CDS group reported increased follow-up rates of $\mathrm{CV}$ risk calculations while seeing patients ( $73 \%$ vs. $28 \%, \mathrm{P}=0.006$ ), being better prepared to discuss $\mathrm{CV}$ risk reduction priorities with patients $(98 \%$ vs. $78 \%, \mathrm{P}=0.03)$, providing accurate advice on aspirin for primary prevention $(75 \%$ vs. $48 \%$, $\mathrm{P}=0.02$ ) and more often discussing $\mathrm{CV}$ risk reduction ( $60 \%$ vs. $30 \%, \mathrm{P}=0.06$ ). CDS users reported that the CDS system improved CV risk factor control (98\%), saved time when talking to patients about CV risk reduction (93\%), efficiently elicited patient treatment preferences $(90 \%)$, was useful for shared decision-making (95\%), influenced treatment recommendations (89\%) and helped initiate CV risk discussions (94\%); $85 \%$ of providers reported that their patients liked CV Wizard.

Conclusion: The CV Wizard CDS system was successfully integrated into the workflow of primary care visits with high sustained use rates, high primary care provider satisfaction, high patient satisfaction and positive impacts on providerreported clinical processes related to $\mathrm{CV}$ risk factor management.

Keywords: clinical decision support, patient engagement
C5-4:

Connecting Primary Care Patients to Community Resources: Lessons Learned From the Development of a New Lay Primary Care Team Role

Clarissa W. Hsu, ${ }^{1}$ Erin Hertel, ${ }^{1}$ June BlueSpruce, ${ }^{1}$ Tyler R. Ross, ${ }^{1}$ Allen Cheadle, ${ }^{1}$ Eric Johnson, ${ }^{1}$ Juno Matthys, ${ }^{1}$ Kelly Ehrlich, ${ }^{1}$ Katie Coleman, ${ }^{1}$ Janice Tufte, ${ }^{1}$ Michelle Robbins, ${ }^{1}$ Paul Fishman ${ }^{1}$

${ }^{1}$ Group Health Research Institute, Seattle, WA

Background/Aims: Social determinants of health play a critical role in both the prevalence and outcomes of a wide range of chronic illnesses. The growing recognition of both the role that social determinants play in population health outcomes and the need to address these requires stronger partnerships between primary care teams and community organizations. In support of this goal, the Learning to Integrate Neighborhoods and Clinical Care (LINCC) project, funded by the Patient-Centered Outcomes Research Institute and implemented at Group Health, convened patients and staff to co-design a new role on the primary care team aimed at helping patients better connect with community resources through one-on-one coaching and follow-up. We implemented this program in three pilot clinics to assess the challenge of incorporating this new team member into primary care practices and the potential impact this program would have if scaled to the entire delivery system.

Methods: We used a mixed-methods approach to document and evaluate the design and implementation of this new role. Data collection included patient surveys, review of electronic health record documentation, site visits, staff interviews and patient focus groups.

Results: We will present a case study on the implementation and evaluation of this role (analysis in progress) with a focus on findings and lessons learned that will enable others to learn from or replicate this pilot. Our findings will include documentation of the patient-centered design and specific scope of work for the role, its integration into the primary care team, and specific tools and approaches developed. We also will report process measures including patient volume, descriptions of the patients served, percentage of patients who completed an action plan, progress made toward accomplishing goals, resources referred and used, and patient satisfaction with the services received.

Conclusion: The LINCC project helped patients connect to community resources by providing deep knowledge of local resources, personalized coaching, goal setting and followup, and an ongoing connection to the primary care team. Preliminary findings highlight the challenges and successes associated with implementing this role and show promising results in terms of patient receptivity and satisfaction with the service.

Keywords: implementation science, primary care teams 
PS2-60:

Integration of Clinical Research Into Health Care Operations: A Comparison of Two Systems

Steven Ziemba, ${ }^{1}$ Brent Ibata ${ }^{2}$

${ }^{1}$ Marshfield Clinic Research Foundation, Marshfield Clinic, Marshfield, WI; '2Cardiac Research Foundation, Sentara Heart Hospital, Norfolk, VA

Background/Aims: Clinical research has become a common component of many health care systems, whether or not they embrace research on a strategic level. However, the nuances of clinical research differ from those of health care, opening the door for inefficiency, poor study outcomes and increased participant risk. We present here a comparative case study on two unaffiliated health care systems that successfully engage in clinical research via different models.

Methods: We examine the clinical research operations of two health care systems, Marshfield Clinic in Marshfield, WI, and Sentara Healthcare in Norfolk, VA. Each system brings unique attributes to the administration of clinical research, including patient population, support infrastructure, physician engagement, regulatory compliance, financial performance, and overall study selection and conduct. Each of these factors are assessed and presented in a comparison of the models each health care system utilizes.

Results: The clinical research operations of the two health care systems described present successful models for the integration of research into health care. Similarities exist, but an analysis of their different approaches will provide valuable information to other sites faced with comparable challenges.

Conclusion: Clinical research is engaged in an environment of contrasts. It shares some common elements such as regulatory requirements and compliance. Drastic differences also can exist between organizations, such as the types of patients served and the engagement of physicians and organization leadership. Understanding how to identify and address these differences can facilitate more successful research overall, providing benefit to all health care systems.

Keywords: clinical research, system model

\section{PS2-61:}

WIC Nutritionist Perspectives on Opportunities and Challenges Regarding Care Coordination With Primary Care Providers for Early Childhood Obesity Prevention

Lisa Bailey-Davis, ${ }^{1}$ Lindsey B. Hess, ${ }^{2}$ Michele Marini, ${ }^{2}$ Jacob Mowery, ${ }^{3}$ Shawnee Lutcher, ${ }^{1}$ Jennifer S. Savage ${ }^{2}$

${ }^{1}$ Obesity Institute, Geisinger Health System, Danville, PA; ${ }^{2}$ Center for Childhood Obesity Research, Pennsylvania State University, University Park, PA; ${ }^{3}$ Center for Health Research, Geisinger Health System, Danville, PA

Background/Aims: Economically disadvantaged children experience disparities in obesity rates despite having numerous points of contact with the broader health care community, namely primary care and the Woman, Infants, and Children (WIC) federal assistance program. Our purpose was to evaluate the perspectives of WIC regarding the potential to coordinate community-based care with the primary care.

Methods: We used a semi-structured interview guide to gather perspectives of WIC nutritionists $(\mathrm{N}=35)$, including state program leaders, through seven focus groups in a MidAtlantic state. Investigators used a grounded-theory approach to independently open-code transcripts. Next, categories were identified and emerging themes reported.

Results: Key themes include: a) WIC is challenged by a clutter of feeding messages from health care, media, family and friends; b) Coordinating care across sectors could enhance patient-centeredness and outcomes; and c) Health information technology strategies are but one piece of a coordinated care system. WIC nutritionists, experts in feeding education, described their role in care but perceive limited power and influence to resolve conflicts in messaging that function as barriers to learning. Bidirectional data sharing (e.g. measured weight and length, immunizations) would be a first step forward toward coordination of care between sectors. Core educational messages, parent written consent, time-sensitivity, defining the scope of practice, relationship building and training are additional issues to be addressed to coordinate care.

Conclusion: WIC perceives opportunity for exponential benefits in reducing message conflict by coordinating care for mother and child-centered needs. Health care provider and patient perceptions should be explored.

Keywords: health system engagement, community providers

PS2-62:

Weight-Related Messages in Well-Child Visits: What Do Teens Desire?

Andrew S. Bossick, ${ }^{1}$ Gwen Alexander, ${ }^{1}$ Tanya Troy, ${ }^{1}$ Heather Olden, ${ }^{1}$ Charles Barone,${ }^{2}$ Andrea E. Cassidy-Bushrow ${ }^{1}$

${ }^{1}$ Department of Public Health Sciences, Henry Ford Health System, Detroit, MI; '2Department of Pediatrics, Henry Ford Health System, Detroit, MI

Background/Aims: With the United States' prevalence of childhood obesity at approximately $17 \%$, well-child visits are a critical time to discuss well-being, physical activity and other concerns. Discussion of healthy weight is a key component of these pediatric visits. Appropriate delivery of weight-related messages to adolescents and their families is critical. Accusatory messages may distance adolescents from health care, whereas nondirect approaches could suggest unhealthy weight is nonproblematic. Feedback on weightrelated messages during the well-child visit may improve health care delivery in this setting.

Methods: Opinions on weight, weight-related messaging, blood pressure and lipid testing knowledge were collected through focus groups with adolescents 14-18 years old. Eligible adolescents had a body mass index $>85$ th percentile and/or a diagnosis of high blood pressure or high cholesterol. A framework matrix method was used for data analysis. 
Results: Four focus groups, stratified by gender, were held (16 participants [9 male]; mean age $15.7 \pm 1.05$ years). Teens expressed that a relationship based on provider trust, built over time, with personal and parental responsibility, are important aspects of the well-child "team." Adolescents voiced strong desire for parental inclusion in health-related discussions. Ideal parental involvement was described as supportive and encouraging to provider recommendations. Teens expressed a lack of knowledge and awareness, along with misconceptions, about health consequences due to unhealthy weight, blood pressure and cholesterol. Providers were described as a resource to discuss weight-related topics; teens expected and welcomed weight-related conversations. Adolescents appreciated casual conversations based on activities and interests, although a serious approach was considered helpful to encourage meaningful changes. Discussions about blood pressure, cholesterol and diabetes were categorized as "scary" and "overwhelming."

Conclusion: Ourdatasuggesta provider-adolescentrelationship built on trust, team work, support and encouragement creates a positive atmosphere for weight-related messages for teens during a well-child visit. Adolescents described long-standing patient-provider histories, suggesting a pediatric medical home concept may be critical in positive discussions of weight. Next steps include analyzing similar parent and provider focus group data and incorporating focus group concepts into a patientcentered, brief, educational intervention for providers focusing on weight-related messages during the well-child visit.

Keywords: well-child visit, qualitative data

\section{PS2-63:}

Leveraging Core Competencies of Collaboration and Innovation to Create a Health System Patient Advisor Resource

\author{
Karen E. Kippen, ${ }^{1}$ Heather Olden ${ }^{1}$
}

\section{${ }^{1}$ Department of Public Health Sciences, Henry Ford Health System, Detroit, MI}

Background/Aims: The Institute of Medicine's Six Aims for Improvement include health care that is patient-centered. With this foundation, Henry Ford has established a unique network rooted in patient, community and health system priorities to create a patient advisor group housed in its public health sciences department. This sustainable health system resource recruits, trains and engages four types of patient/family advisors (PFA): health system advisors, research advisors, e-advisors, and focus group advisors. These advisors are integrated into patient-centered outcomes research, quality improvement projects and service excellence teams, with an increasing demand for more advisors throughout the system. Methods: Located in a vertically integrated health system, our innovation is making patient-centered approaches central to our research and to the health care the system provides. By leveraging our core competencies of collaboration and innovation, this group was built on the foundation of shared patient, community and health system interests with an overall goal to create advisory councils at all system locations. This framework recruits and trains a representative group of PFAs and embeds them in projects where their perspective is incorporated into patient care, work processes and current/future research. Through collaboration with PFAs, researchers, community leaders, system senior leadership, and clinical, operational and corporate support services, we are continuously evaluating our processes, learning and making improvements.

Results: A key success story example is our process improvement project for head and neck cancer patients begun as a PFA council to develop a patient resource guide, which evolved using our engagement model into a Patient-Centered Outcomes Research Institute-funded award to study qualityof-life outcomes. Total PFAs $=110$, total system improvement teams $=8$, and total research project teams $=5$.

Conclusion: Developing a PFA resource has provided three key benefits. Researchers benefit by executing patientcentered research through engagement of advisors in all phases of projects, including the development of new grant ideas. PFAs benefit by sharing their stories with providers to create evidence-based improvements and results. Finally, learning how we can improve our health system through the eyes of patients can improve outcomes through safe, effective and patient-centered care processes.

Keywords: patient advisor, engagement

PS2-64:

Formalizing and Optimizing Patient Engagement in a Large Health Care System

\author{
Janet L. Williams, ${ }^{1}$ Alanna Kulchak Rahm, ${ }^{1}$ F. Daniel \\ Davis $^{2}$ \\ ${ }^{1}$ Genomic Medicine Institute, Geisinger Health System, \\ Danville, PA; '2Bioethics, Geisinger Health System, \\ Danville, $P A$
}

Background/Aims: Patient engagement and patientcenteredness have become catchphrases used as core principles in reference to the Affordable Care Act, the Learning Healthcare System workshop, value-driven care and the Patient-Centered Outcomes Research Institute. Patient engagement is not a new concept; in 2001, the Institute of Medicine defined patientand family-centered care as care based on continuous healing relationships, customized according to patient needs and values, patient control, information equipoise, transparency and anticipation of patient needs. Increased attention is also being paid to include patient-defined outcomes, demonstrated shared decision-making and patient assessed value in their care as benchmarks for reimbursement.

Methods: At Geisinger Health System, we have developed a framework to guide patient engagement across the continuum of care and in research and discovery to meet the strategic aim of enhancing the experience and quality of care for patients and their families. We have launched several initiatives and working groups to improve engagement of patients, families and clinical stakeholders to continue evolving as a learning health care system. Initiatives include assessment of existing expertise to assist in engagement across the research continuum, multiple 
advisory boards and development of purposeful community engagement activities. Workgroups developed include projectspecific patient advisory committees, a patient engagement workgroup comprised of stakeholders across research and health care operations, and workgroups to design specific engagement tasks such as symposia and knowledge assessments regarding the learning health care system environment.

Results: We will present the overarching framework and strategies developed by the patient engagement workgroup and share materials and strategies employed to encourage meaningful patient engagement in study design, measures, analysis and conclusions. Strategies include pre-engagement activities, identifying patient research partners, supporting patient partners and defining the role of patient partners in dissemination and implementation.

Conclusion: Effective patient and family engagement in research depends on adequate technical, programmatic and human resources that collectively constitute an integral infrastructure.

Keywords: patient engagement, learning health care system

\section{PS2-65:}

\section{Building a Patient Stakeholder Council and Patient Partner Registry: Resources for Patients and Researchers}

\section{Sarah Madrid, ${ }^{1}$ Leslie Wright, ${ }^{1}$ Heather Tabano ${ }^{1}$ \\ ${ }^{1}$ Institute for Health Research, Kaiser Permanente Colorado, Denver, CO}

Background/Aims: Patient input is critical to ensuring that research studies and their outcomes are relevant to patient priorities and needs. Research teams need a way to quickly identify appropriate patient partners for engaged research, and patients anxious for a voice in research need a way to access research teams and organizations. The Patient Stakeholder Council (PSC) and Colorado Patient Partners in Research (CoPPiR) network address these needs. The PSC broadly informs research agendas while the CoPPiR network connects patients with research teams, giving teams a way to rapidly identify, contact and recruit patient partners with specific characteristics.

Methods: Kaiser Permanente Colorado's Institute for Health Research, Denver Health's Center for Health Systems Research (CHSR), and the CHSR's Community Advisory Panel are partnering equally to create these resources, and methods will be the focus of this presentation. All three partners defined the purpose and goals of the PSC and are collaborating to recruit and train all members of the PSC. Simultaneously, the three partners are building the CoPPiR network infrastructure. The CoPPiR website provides a centralized means of providing information about the network, facilitating recruitment of members and responding to requests from individual research teams. Both resources explicitly prioritize recruitment of members reflecting the demographic, socioeconomic and racial/ ethnic characteristics of these two health system memberships. Results: The outcomes of the successful creation of both the PSC and the CoPPiR network include improving research teams' abilities to write patient-centered research proposals and conduct studies whose outcomes are more relevant to patient populations. Equally important is the empowerment of patient research partners to influence overall research agendas and, in individual research studies, to voice concerns, insights and preferences of patients.

Conclusion: Tackling the needs of both patients and researchers in the development of engagement resources provides a winwin in the effort to increase the quality and meaningfulness of patient-centered outcomes research. Further, establishing both a broadly focused panel and a mechanism for matching patient partners with specific research teams to provide deeper insights on a particular disease burden or health condition effectively addresses distinct needs of research organizations and provides a model for replication elsewhere.

Keywords: patient engagement, patient-centered outcomes research

PS2-66:

Evidence-Based Practice Integration Designed to Optimize Patient Care and Outcomes to Achieve the Triple Aim

\section{Jeffrey Hansen, ${ }^{1}$ Camille Haycock, ${ }^{1}$ Elizabeth M. Evans ${ }^{2}$ \\ ${ }^{1} \mathrm{CHI}$ Evidence-Based Practice/Care Continuum, CHI Institute for Research and Innovation, Englewood, $\mathrm{CO} ;{ }^{2} \mathrm{CHI}$ Foundation, $\mathrm{CHI}$ Institute for Research and Innovation, Englewood, CO}

Background/Aims: Evidence-basedpractice(EBP)integration at Catholic Health Initiatives (CHI) is a multidisciplinary collaborative approach to optimize patient care and outcomes. The process and structure for $\mathrm{EBP}$ integration at $\mathrm{CHI}$ involves translating research-based, evidence-supported knowledge into clinical practice and process flows. In 2011, CHI founded the National EBP Advisory Council (NEBPAC) to facilitate multidisciplinary collaborative identification of and support for organizational EBP initiatives. The first organizational EBP initiative addressed by the NEBPAC was to review published EBP models for facilitated development of a custom organizational EBP model and algorithm that would align with CHI's core values. In 2012, a five-phase custom EBP Model $^{\circ}$ and Algorithm $^{\circ}$ was deployed by the NEBPAC to guide EBP integration across the organization.

Methods: In 2013, the NEBPAC facilitated multidisciplinary development of 13 new organizational EBP toolkits with inclusion of referential hyperlinks to resources that provide data-driven decision-making and directional guidance for addressing identified organizational clinical quality improvement opportunities. Potential considerations for accurately identifying organizational clinical quality improvement opportunities include the following: a) appraisal of organizational culture and readiness for EBP integration, b) provider/clinician/staff awareness of foundational clinical practice guidelines published by national organizations and/or regulatory agencies, c) accuracy of clinical documentation with ease of electronic abstraction for analysis and regulatory reporting purposes, and d) evaluation of clinician resourcing based on patient acuity designed to improve 
clinical quality outcomes.

Results: Potential results associated with EBP integration include the following: a) improved patient outcomes with reduced cost of care involving complications, length of stay or mortality; b) increase in government-sponsored incentives with avoidance of penalties; and c) achievements in accreditations and designations for excellence.

Conclusion: CHI's approach to EBP integration allows for data-driven decision-making that promotes clinical quality improvement. This approach contributes to achievement of the Triple Aim.

Keywords: evidence-based practice, Triple Aim

\section{VDW METHODS AND PROCESSES}

B2-1:

\section{Transition to ICD-10 Diagnoses and Procedures in the HCSRN Virtual Data Warehouse}

Don Bachman, ${ }^{1}$ Michael J. Allison, ${ }^{1}$ Alan E. Bauck, ${ }^{1}$ Catherine Cleveland, ${ }^{1}$ Phil Crawford, ${ }^{1}$ Suzanne Gillespie, ${ }^{1}$ Mary Ann McBurnie, ${ }^{1}$ Mark C. Hornbrook, ${ }^{1}$ Alexandra M. Varga, ${ }^{1}$ Xiahai Yang, ${ }^{1}$ Roy Pardee $^{2}$

${ }^{1}$ Center for Health Research, Kaiser Permanente Northwest, Portland, OR; ${ }^{2}$ Group Health Research Institute, Seattle, WA

Background/Aims: Federal regulations require that all U.S. health care providers transition from ICD-9 to ICD-10 for diagnosis and hospital procedure coding on Oct. 1, 2015. The changes between the two versions are dramatic; the new version expands the number of diagnosis codes from about 13,000 to 68,000 and increases the number of procedure codes from approximately 4,000 to 90,000 . These changes require programmers to modify their file-building software when creating the diagnosis and procedure tables in the Health Care Systems Research Network (HCSRN) Virtual Data Warehouse (VDW). We will describe the transition to ICD-10 diagnostic and procedure data in the VDW as well as development of data quality reports to check on completeness of ICD-10 conversion at the participating HCSRN sites.

Methods: The ICD-10 transition team from Kaiser Permanente Northwest's Center for Health Research worked with subject matter experts and research project staff to develop categories of ICD-9 and ICD-10 diagnosis and procedure codes that all signify the same general condition (e.g. diabetes, congestive heart failure). The team then developed a program that plots daily counts of patients with one or more of the codes in each category. These plots show the consistency of counts of patients within each category before and after the transition date for the two ICD coding systems. This program will be distributed to the HCSRN sites to use as an early warning system for issues in the transition and to validate the VDW tables. Sites shared their results with the HCSRN Utilization Work Group.

Results: We expect to find that the counts of patients with specific ICD diagnosis and procedure categories are consistent before and after the transition to ICD-10 for most HCSRN sites. We also believe the monitoring reports will be useful for identifying issues early, which should prompt corrections to the VDW before research teams begin using ICD-10 data.

Conclusion: We expect that HCSRN sites will use these reports to validate ICD-10 diagnostic and procedure data and demonstrate these data are ready to be used in the VDW. Research teams will need to create crosswalks between ICD-9 and ICD-10 data if their projects span Oct. 1, 2015.

Keywords: ICD-10 diagnoses, ICD-10 procedures

B2-2:

Application of Propensity Score Models in Observational Studies Using VDW Data

Nikki M. Carroll ${ }^{1}$

\section{${ }^{1}$ Institute for Health Research, Kaiser Permanente Colorado, Denver, CO}

Background/Aims: Treatment effects from observational studies may be biased since the patients were not randomly allocated to a treated or untreated group. Propensity score methods are increasingly being used to address this bias. The propensity score is the probability of treatment assignment conditional on observed baseline characteristics. After appropriately adjusting for the propensity score, the distribution of observed baseline covariates will be similar between treated and untreated patients.

Methods: The estimation of propensity scores and how they can help in adjusting the treatment effect for differences in baseline imbalances are reviewed. Variable selection for the propensity score model, balancing the propensity score, and suggestions on what to do when the propensity score does not balance also are reviewed. The strengths and limitations of each of the different methods of propensity score adjustment are presented (e.g. matching, covariate adjustment, stratification, inverse probability of treatment weighted [IPTW], stabilized IPTW) as well as recommendations for different sensitivity analyses to look at the magnitude of hidden biases. Finally, suggestions are provided on what to present in the report of the final analysis.

Results: Propensity score methods are illustrated by estimating the effect of angiotensin-converting enzyme inhibitor (ACEI) and angiotensin receptor blocker (ARB) use in stage IIIb/IV non-small cell lung cancer (a project funded by Kaiser Permanente's Center for Effectiveness \& Safety Research). All steps of the propensity score methods used to analyze the effect of ACEI and/or ARB antihypertension treatment on survival in advanced stage non-small cell lung cancer patients are described.

Conclusion: Although propensity scores cannot control for unobserved or unmeasured confounding, propensity score analyses can address residual confounding by simulating randomized control trials and are a tool one can use when comparing two treatment groups.

Keywords: propensity score, observational study methods 
B2-3:

\section{Mapping Medication Orders to RxNorm Concepts}

Jenny Staab, ${ }^{1}$ Andy Karnopp ${ }^{1}$

${ }^{1}$ Center for Health Research, Kaiser Permanente Northwest, Portland, OR

Background/Aims: There is a multitude of coding or classification systems for medications. While dispensed medications have traditionally been identified using National Drug Codes (NDCs), there has been no equally universal vocabulary for ordered medications. However, RxNorm, a nonproprietary drug terminology system developed by the National Library of Medicine, has become increasingly popular. Both PCORnet and the Observational Medical Outcomes Partnership's Common Data Models have adopted RxNorm as a standard vocabulary for medications. RxNorm concepts range in specificity from branded packaged drugs to simple ingredients and can be linked to many proprietary and nonproprietary vocabularies such as NDCs or Medi-Span Generic Product Identifier (GPIs). We developed a method to map drugs from drug names and other drug terminologies to RxNorm concept unique identifiers (CUIs).

Methods: Data on ordered medications were extracted from Kaiser Permanente Northwest's Clarity database. These data contained text strings identifying drugs and generic ingredient names as well as codes from different drug vocabularies including RxNorm, GCN Sequence Numbers, GPIs and NDCs. These vocabularies were available for overlapping subsets of the medications list. We used directly available CUIs and crosswalks linking other vocabularies to RxNorm. For drugs that could not be mapped satisfactorily, we also employed approximate string matching using RxMix. Potential concept matches were ranked using a scoring algorithm and the best match chosen. CUIs deemed highly reliable, such as those provided directly from Clarity or deemed a perfect match by RxMix, scored higher. CUIs scored lower if they were derived from obsolete NDCs or scored poorly on RxMix.

Results: We found CUIs for over $95 \%$ of orders; $75 \%$ of matches were fully specified drugs, the remaining were mostly ingredients. About $10 \%$ of CUIs were found only via string match, with an average confidence score of $75 \%$.

Conclusion: There are multiple ways to map medications to RxNorm concepts. Using direct crosswalks based on standardized terminology is preferable, as it affords higher certainty. However, approximate string matching can provide mappings for medications without available standardized terminology. It also can improve data quality by mapping to more specific RxNorm concepts and supporting validation of the direct mapping results. The adopted level of certainty for inclusion in the dataset should be well documented.

Keywords: RxNorm, pharmacy
B2-4:

The CHI Institute for Research and Innovation Virtual Data Warehouse - A Case Study of Health Operations Partnership in Development of Data Assets

\author{
Lela McFarland, ${ }^{1}$ Deborah O'Dell, ${ }^{2}$ Matthew Taylor, ${ }^{3}$ \\ Laurie Jenson ${ }^{4}$
}

${ }^{1}$ Clinical Data Research Management, $\mathrm{CHI}$ Institute for Research and Innovation, Englewood, CO; ${ }^{2}$ Business Intelligence, $\mathrm{CHI}$ Institute for Research and Innovation, Englewood, CO; ${ }^{3}$ Clinically Integrated Networks, $\mathrm{CHI}$ Institute for Research and Innovation, Englewood, CO; ${ }^{4}$ National Oncology Service Line, CHI Institute for Research and Innovation, Englewood, CO

Background/Aims: The Virtual Data Warehouse (VDW) was created as a mechanism for producing comparable data across different health care organization sites for purposes of proposing and conducting research. At the core of the VDW are a series of standardized file definitions. The content areas and data elements included in the VDW data specification are commonly required for research studies.

Methods: The VDW specifications were used as a foundational guide for the development of an organizationally shared Catholic Health Initiatives (CHI) enterprise data store. This shared organizational data capability became the upstream source from which the CHI Institute for Research and Innovation was able to establish a VDW, along with other research industry standard models such as Observational Medical Outcomes Partnership or Informatics for Integrating Biology and the Bedside databases.

Results: In order to collaboratively build a repository with our parent health care organization, several key elements needed to be established. First, effective governance structures needed to be employed including cooperative buy-in into shared timelines and priorities. Second, VDW specifications had to be translated to be more easily consumable by a nonresearch audience. Third, organizational reporting needs were compared against VDW model capabilities to understand their relevancy beyond research analytics and identify enhancements that were needed to make base VDW model capabilities to broaden the applicable audience of the model. Fourth, cooperative approaches to understanding data quality had to be developed. Finally, the process of ongoing governance and prioritization of improvements and expansions were established.

Conclusion: The approach of research analytics sharing in the development of organizational health operations data assets has brought a number of benefits in establishing a VDW. However, the shared approach also highlighted areas where additional work is needed to establish synergy between research analytics and health operations analytics efforts.

Keywords: Virtual Data Warehouse, analytics 
PS2-67:

\section{Estimating Generic Drug Utilization With Electronic Health Records From a Mixed-Payer Ambulatory Health Care Delivery System}

\author{
Robert J. Romanelli, ${ }^{1}$ Vani Nimbal, ${ }^{1}$ Trang Le,${ }^{2}$ Jodi B. \\ Segal ${ }^{3}$
}

${ }^{1}$ Palo Alto Medical Foundation Research Institute, Palo Alto Medical Foundation for Health Care, Research and Education, Palo Alto, CA; ${ }^{2}$ Department of Clinical Pharmacy, University of California, San Francisco, San Francisco, CA; ${ }^{3}$ Department of Medicine, Johns Hopkins School of Medicine, Baltimore, MD

Background/Aims: Generic drug utilization in an outpatient setting is typically measured through reconciled pharmacy claims data; however, not all health care systems have access to these data for their populations. We sought to develop and validate an automated algorithm to estimate generic drug utilization with electronic health records (EHR) data.

Methods: We chose 25 therapeutic classes to study due to their potential for poor generic uptake. We developed a generic utilization algorithm using EHR prescribing data (i.e. medication name and indicator of a dispense-as-written request) from a mixed-payer health care system in northern California in 2013. These data were combined with information on generic product availability from Drugs@FDA and Redbook ${ }^{\mathrm{TM}}$ at the time prescriptions were written. The algorithm was validated in a cohort of ambulatory managed-care beneficiaries for whom prescribing data and pharmacy claims data (i.e. gold standard) are both available. An estimated generic utilization rate (GUR) across therapeutic classes was calculated by applying the algorithm, and its performance was assessed. We next applied the algorithm to electronic prescribing data from a cohort of non-managed-care beneficiaries for whom pharmacy claims data are not available.

Results: We identified 20,750 managed-care beneficiaries with an electronic prescription for a drug product in the therapeutic classes of interest in 2013 and a corresponding pharmacy claim. We also identified 119,153 non-managedcare beneficiaries with electronic prescriptions for products in the same therapeutic classes. Managed-care and nonmanaged-care beneficiaries were similar with respect to age, sex and race/ethnicity. Applying the algorithm, we estimated a GUR of $58.7 \%$ among managed-care beneficiaries; the actual GUR based on pharmacy claims was $57.2 \%$. Sensitivity (96\%) and specificity (94\%) of the algorithm were high, and total percent agreement was $96 \%$. In the cohort of non-managedcare beneficiaries, a similar GUR was estimated (56.1\%).

Conclusion: A generic utilization algorithm that combined EHR data with information on generic product availability was validated and performed well in estimating the GUR for outpatient prescriptions in a cohort of managed-care beneficiaries, independent of pharmacy claims data. Health care delivery systems may apply the methods described herein to quantify generic utilization in their ambulatory populations for quality improvement and research initiatives.

Keywords: generic drugs, electronic health records
PS2-68:

Using SmartTools to Capture Structured Data From Text: The LINCC Case Study

Tyler R. Ross, ${ }^{1}$ June BlueSpruce, ${ }^{1}$ Erin Hertel, ${ }^{1}$ Woody

Favinger, ${ }^{1}$ Clarissa $\mathrm{Hu}^{1}$

${ }^{1}$ Group Health Research Institute, Seattle, WA

Background/Aims: The widespread adoption of modern electronic heath records (EHR) has digitized medical information previously captured on paper. However, research and analysis requires use of narrative text and involves resource intensive chart review and/or natural language processing. An emerging alternative solution is to use SmartTools in Epic's EHR. We present a case study of a research project using linked SmartTools in Epic to create structured data from text and describe the strengths and challenges of these tools.

Methods: The Learning to Integrate Neighborhoods and Clinical Care (LINCC) study collaborated with clinic staff to develop SmartPhrases with embedded SmartLists to be used by community resources specialists (CRS). The CRS were trained to use these SmartPhrases to document their patient interactions and capture critical information for process improvement and evaluation. The LINCC study worked with information technology (IT) to preserve SmartList selections in discrete fields in the EHR reporting database. The study team retrieved both the CRS notes and the linked SmartList selections and created an abstraction system in which values for relevant variables could be coded.

Results: The CRS began documenting patient encounters using SmartPhrases before their embedded SmartLists were linked to the reporting database. As a result, only free-text data were available from the early part of the study period. As the CRS position evolved, the SmartPhrases were modified and new SmartLists required linkage. This iterative process created inconsistency in measurement over the study period and occasional loss of information due to linkage timing. Inconsistency in how SmartPhrases were used within and across the CRS led to further measurement error. Ultimately, the team abstracted variable values from free-text notes for the final set of measures for analysis.

Conclusion: In this case study, despite clinic leadership and IT support and the opportunity to use SmartTools, abstraction from each CRS note was necessary to obtain some analytic measures. Although SmartTools did not eliminate the need for note review, they helped structure documentation, reduced the number of abstracted measures and provided supplemental information that aided the abstraction process. Extensive planning, coordination and training are critical for SmartTools to be used effectively for gathering research quality metrics.

Keywords: informatics, electronic health records 
PS2-69:

Design, Implementation, and Response Rates From an Online Patient Survey to Assess Genitourinary Symptoms and Related Health Care Experiences of Postmenopausal Women

Joanna Bulkley, ${ }^{1}$ Ashley Stoneburner, ${ }^{1}$ Michael Leo, ${ }^{1}$ Amanda Clark, ${ }^{2}$ Kate Beadle, ${ }^{2}$ Kimberly K. Vesco ${ }^{1}$

${ }^{1}$ Center for Health Research, Kaiser Permanente Northwest, Portland, OR; '2Obstetrics and Gynecology, Kaiser Permanente Northwest, Portland, OR

Background/Aims: Nearly 50\% of postmenopausal women experience symptoms related to genitourinary syndrome of menopause (GSM), including vulvovaginal dryness and irritation, painful intercourse and urinary incontinence. As part of a clinician-focused intervention to improve diagnosis and management of GSM at Kaiser Permanente Northwest, we conducted an online survey of women with a wellwoman visit to primary care and obstetrics/gynecology (OB/ GYN) to assess patient vulvovaginal, urinary and sexual symptoms and related health care experiences. Our goal was to maximize patient participation while minimizing staffing requirements.

Methods: Electronic communication was used for all contact with patients, including recruitment, consent, eligibility screening, survey and thank you gift cards. REDCap was used for everything except e-gift card (\$5) delivery, which required a separate system. We sent an email invitation to all women $\geq 55$ years old with a well-woman visit to primary care or $\mathrm{OB} / \mathrm{GYN}$ during the previous week and an email address on file. Eligible patients were identified from the electronic medical record and uploaded to REDCap weekly. An initial recruitment email, which included a link to the survey, was sent each Tuesday morning. Patients who did not respond were re-sent the email the next Monday afternoon. Patients who clicked the survey link were taken to the REDCap site, which explained the study and contained the IRB-required consent language. Participants who actively agreed to participate then completed an eligibility screening page and, finally, the survey. For security, only one-time access was allowed to the survey. E-gift cards were sent weekly to all new respondents. REDCap and SAS software reports were run weekly to track recruitment patterns and watch for data anomalies.

Results: Email addresses were found for $80 \%$ of eligible women $(5,961 / 7,483)$. The overall response rate was $25 \%$ $(1,483 / 5,961)$ and varied across the 18 randomized clinics from $19 \%$ to $34 \%$. The response rate was lower for women $\geq 75$ years old $(18 \%$ vs. $26 \%$ for $<75)$. The active refusal rate was $2 \%$.

Conclusion: Electronic surveys can successfully be used at relatively low cost, even for topics that are sensitive in nature. Lessons learned and copies of recruitment, consent, screening and survey materials as well as recruitment reports will be presented.

Keywords: patient survey methodology, genitourinary syndrome of menopause
PS2-70:

A Novel Method for Estimating Transgender Status Using EMR Data

Douglas W. Roblin, ${ }^{1}$ Michael Goodman, ${ }^{2}$ Lee Cromwell, ${ }^{1}$ Laura Schild, ${ }^{1}$ Enid Hunkeler, ${ }^{3}$ Virginia Quinn, ${ }^{3}$ Brandi Robinson, ${ }^{1}$ Hayley Braun, ${ }^{2}$ Rebecca Nash, ${ }^{2}$ Josh Gerth, ${ }^{2}$ Joshua Barzilay, ${ }^{4}$ Vin Tangpricha ${ }^{5}$

${ }^{1}$ Center for Clinical Outcomes Research, Kaiser Permanente Southeast, Atlanta, GA; ${ }^{2}$ Rollins School of Public Health, Emory University, Atlanta, GA; ${ }^{3}$ Division of Research, Kaiser Permanente Northern California, Oakland, CA; ${ }^{4}$ Endocrinology, Kaiser Permanente Southeast, Atlanta, GA; ${ }^{5}$ Department of Medicine, Emory University, Atlanta, GA

Background/Aims: "Transgender" broadly describes individuals who either do not identify with their biological sex or do not conform to binary gender categories. Studies of transgender persons are impeded by lack of consistent terminology or coding to identify the population. Thus, transgender prevalence, direction of change (male-to-female [MTF] or female-to-male [FTM]) and health outcomes are not well known. We describe a novel algorithm for identifying a cohort of transgender persons from diverse electronic medical record (EMR) system data sources.

Methods: Provider visits for Kaiser Permanente Georgia members enrolled between 2006 and 2014 were collected from EMR databases. The method to build a cohort involved an SAS software algorithm, which scanned these databases for relevant ICD-9 codes and presence of specific keywords in digitized provider notes. The first step was to identify transgender status (e.g. keywords "transgender" or "gender identity") and the second step to determine natal sex (e.g. keywords of "ovaries" or "testes") given confirmed transgender status. Trained reviewers confirmed transgender status and, if confirmed, determined natal sex from a limited, focused review of keyword-containing text strings $( \pm 50-100$ characters around the keyword, stripped of protected health information). Accuracy of identification and proportions of MTF and FTM subjects were computed, along with $95 \%$ confidence intervals (CI).

Results: Of 823,104 members, 271 were identified as possibly transgender by the SAS algorithm: 137 through keywords only, 25 through ICD-9 codes only, and 109 through both ICD9 and keywords. Of these 271, 185 (68\%, 95\% CI: 62-74) were confirmed as transgender: $62(45 \%, 95 \%$ CI: 37-54), 14 (56\%, 95\% CI: 35-75), and 109 (100\%; 95\% CI: 96-100) for keywords only, ICD codes only, and both, respectively. Of the 185 confirmed transgender persons, 95 (51\%, 95\% CI: 44-59) were MTF and 76 (41\%, 95\% CI: 34-49) were FTM; natal sex remained unknown for the remaining 14 (8\%, 95\% CI: 4-13).

Conclusion: This method for support of research on transgender persons is low cost, rapid and valid - saving substantial time and cost that would have been required of review of nearly 1 million medical records. The overall approach, and the SAS algorithm, can be easily transferred to other health care systems.

Keywords: disparities, transgender 
PS2-71:

VDW Data Sources: CHI Institute for Research and Innovation

Lela L. McFarland, ${ }^{1}$ John Weeks, ${ }^{1,2}$ Penne Perry, ${ }^{3}$ Rebecca Towne ${ }^{3}$

${ }^{1}$ Clinical Data Research Management, $\mathrm{CHI}$ Institute for Research and Innovation, Englewood, CO; ${ }^{2}$ Perficient Inc., Englewood, CO; ${ }^{3}$ National Oncology Service Line, $\mathrm{CHI}$ Institute for Research and Innovation, Englewood, CO

Background/Aims: The Virtual Data Warehouse (VDW) was created as a mechanism for producing comparable data across sites for purposes of proposing and conducting research. It is "virtual" in the sense that the data remain at the local sites rather than at a centralized data coordinating center.

Methods: At the core of the VDW are a series of standardized file definitions. Content areas and data elements that are commonly required for research studies are identified, and data dictionaries are created for each of the content areas, specifying a common format for each of the elements variable name, label, description, code values and value labels. Local site programmers have mapped the data elements from their HMO's data systems into this standardized set of variable definitions, names and codes as well as onto standardized SAS software file formats. This common structure of the VDW files enables a SAS analyst at one site to write one program to extract and/or analyze data at all participating sites.

Results: The CHI Institute for Research and Innovation (CIRI) local implementation of the VDW contains detailed medical information on Catholic Health Initiatives patients. The initial establishment of the VDW data files focused on the development of patient demographics, provider demographics, code mapping capabilities, utilization data, chem laboratory data, vital signs, social history and tumor data sets. Phase I also included the development of a non-VDW dataset for pathology labs. Phase II development focused on pharmacy, census, decedent and enrollment data as well as the non-VDW dataset for clinical notes.

Conclusion: This poster demonstrates the range of data sources used at CIRI to feed information into our local implementation of the VDW datasets.

Keywords: Virtual Data Warehouse, standardization

\section{PS2-72:}

Identifying Race/Ethnicity Data via Natural Language Processing Among Women in a Uterine Fibroid Cohort Study

Jane R. Grafton, ${ }^{1}$ Onchee Yu, ${ }^{1}$ David Carrell, ${ }^{1}$ Susan Reed, ${ }^{1}$ Renate Shulze-Rath, ${ }^{2}$ Kelly Hansen, ${ }^{1}$ Delia Scholes ${ }^{1}$

${ }^{1}$ Group Health Research Institute, Seattle, WA; ${ }^{2}$ Bater Health Care, Leverkusen, Germany

Background/Aims: Uterine fibroids are associated with morbidity including abnormal bleeding, anemia, pelvic/bladder symptoms and adverse reproductive outcomes. Symptomatic fibroids may affect $25 \%$ of women in their late 40 s. Race is among the most consistent risk factors known. The Uterine Fibroid Study aims to use automated data to estimate fibroid incidence rates/trends during 2005-2014 in a retrospective cohort of women at Group Health. Race/ethnicity captured from automated structured data has improved yet remains incomplete, particularly with use of retrospective data.

Methods: The study included women 18-65 years old without hysterectomy, continuously enrolled with evidence of encounter in 3 years before study entry. Incidence estimates required absence of fibroid history. We collected fibroid diagnoses, demographics and other data from the Virtual Date Warehouse (VDW). VDW demographic race/ethnicity data is sourced from data collected from Group Practice patients, at time of encounter, via entry in the electronic health record. Additionally, Group Health collects race/ethnicity data from breast cancer screening program and tumor registry data. To complement traditional structured race/ethnicity data from VDW, we augmented with race/ethnicity extracted from freetext clinical notes via natural language processing (NLP). Our NLP system used a rule-based dictionary look-up approach to identify common terms used to describe patient race/ethnicity and custom rules to disambiguate race/ethnicity terms that also have other clinical meanings (e.g. the term "white" in "54-year-old white female" as opposed to "Her white blood cell count improved"). We conducted a partial validation of the NLP system in a sample of patients with known structured race/ethnicity data.

Results: Prior to amending race/ethnicity data with NLP, in the cohort of 277,821 women, $37.4 \%$ had race/ethnicity unknown. Fibroid incidence rates (per 10,000 person-years) were 156 for Hispanics, 133 for whites, 265 for AfricanAmericans, 152 for Asian/Pacific Islanders and 108 for unknown race. NLP work on identifying race/ethnicity in the unknown race group is ongoing and results are pending.

Conclusion: Race/ethnicity is an important risk factor for a number of conditions, including uterine fibroids. Improving capture of race/ethnicity from available automated data sources potentially could improve accuracy of research findings and enhance patient care by providing a better understanding of the burden of disease in subgroups of affected patients.

Keywords: race, natural language processing

PS2-73:

The Learning Healthcare (Data) System: Virtual Data Warehouse Data Capture Revisited

Roy E. Pardee, ${ }^{1}$ Don Bachman, ${ }^{2}$ Mark C. Hornbrook, ${ }^{2}$ Catherine R. Cleveland, ${ }^{2}$ Priyam Mathur, ${ }^{3}$ Dan $\mathrm{Ng}^{4}{ }^{4}$ Susan M. Aumer, ${ }^{5}$ William H. Harding, ${ }^{6}$ Celia Jordan, ${ }^{7}$ Jeremy Meier, ${ }^{8}$ Carmen C. Wong, ${ }^{9}$ Brian A. Hoch ${ }^{10}$

${ }^{1}$ Group Health Research Institute, Seattle, WA; ${ }^{2}$ Center for Health Research, Kaiser Permanente Northwest, Portland, OR; ${ }^{3}$ Meyers Primary Care Institute, Worcester, MA; ${ }^{4}$ Division of Research, Kaiser Permanente Northern California, Oakland, CA; ${ }^{5}$ HealthPartners Institute for 
Education and Research, Bloomington, $\mathrm{MN} ;{ }^{6}$ Institute for Health Research, Kaiser Permanente Colorado, Denver, CO; ${ }^{7}$ Mid-Atlantic Research Institute, Kaiser Permanente Mid-Atlantic States, Rockville, MD; ${ }^{8}$ Essentia Institute of Rural Health, Essentia Health, Duluth, MN; ${ }^{9}$ Center for Health Research, Kaiser Permanente Hawaii, Honolulu, HI; ${ }^{10}$ Marshfield Clinic Research Foundation, Marshfield Clinic, Marshfield, WI

Background/Aims: At the 2014 HCSRN annual meeting, Bachman and colleagues presented an excellent investigation into rates of encounters and drug fills at Virtual Data Warehouse (VDW) sites in order to evaluate (among other things) the VDW enrollment file's "OUTSIDE UTILIZATION" field, which purported to flag periods during which complete data capture of either pharmacy or encounter data was suspect. That investigation revealed serious problems with the flag, calling its usefulness into question. Taking this to heart, the VDW enrollment workgroup proposed removing this field and adding a suite of six new flags intended to express confidence in the capture of pharmacy, laboratory, outpatient encounter, inpatient encounter, tumor and electronic medical record data individually. These flags are assigned by local VDW analysts on the basis of their knowledge of data capture limitations at their respective sites for identifiable subgroups of patients. VDW programs were written and tested for creating these new data incompleteness variables. All HCSRN sites were invited to run these programs and share their results.

Methods: Modeled after Bachman et al.'s work, we calculated rates of pharmacy fills, lab results, encounters, tumor records and vital signs by the appropriate new flag. We then plotted these rates over time to see whether in fact the people/periods flagged as having suspect data capture did in fact have lower rates compared to those who/that were not.

Results: At the sites that implemented the flags, data capture rates generally varied in line with expectations - suspected incomplete groups had markedly lower rates. Of the six flags, "incomplete_rx" saw the best implementations, with all seven implementing sites showing clear distinctions between people whose data capture was suspect and those for whom it was not. "Incomplete tumor" had the most variable implementations, with clear distinctions at some sites but not others.

Conclusion: On balance, the new flags stand to improve the quality of data-based research in the HCSRN. Projects needing to define populations at risk of exposure to particular pharmacy fills, tumors or lab result values, for example, would do well to use the new flags to screen out people for whom exposure risk may not be completely captured.

Keywords: data capture, population-based research
PS2-74:

Validation of the Automated Diagnosis, Intractability, Risk, Efficacy (DIRE) Opioid Risk Assessment Tool

Irina V. Haller, ${ }^{1}$ Colleen M. Renier, ${ }^{1}$ Paul Hitz, ${ }^{1}$ Jeanette A. Palcher, ${ }^{1}$ Thomas E. Elliott ${ }^{2}$

${ }^{1}$ Essentia Institute of Rural Health, Essentia Health, Duluth, MN; ${ }^{2}$ HealthPartners Institute for Education and Research, Minneapolis, MN

Background/Aims: Despite the drastic increases in prescription opioid misuse and abuse, risk assessment for aberrant drug-related behaviors prior to initiating opioid therapy for chronic noncancer pain management continues to be underutilized in clinical practice. The purpose of the study was to investigate availability of data elements in the electronic health record that could be used to assess risk for aberrant drug-related behaviors with the automated Diagnosis, Intractability, Risk, Efficacy (DIRE) opioid risk assessment tool.

Methods: DIRE is a 7-item tool usually administered by a clinician and used to predict efficacy of analgesia and patient compliance with long-term opioid therapy. Each factor is rated from 1 (least favorable case) to 3 (more favorable case for opioid prescribing). The total score is used for risk stratification with scores $<14$ being an unsuitable candidate and scores $\geq 14$ being possible candidate for opioid therapy. The validation of the automated process versus clinicianadministered rating was conducted using kappa analysis and test characteristics (sensitivity, specificity, positive and negative predictive values).

Results: We developed structured data queries, natural language processing (NLP) algorithms for unstructured data, and data mapping strategies to populate the DIRE for a cohort of chronic noncancer pain patients who were on long-term opioid therapy and who had a clinician-administered DIRE documented in the electronic health record prior to signing the most recent opioid agreement. We used ICD-9 diagnosis codes and NLP to populate diagnosis, psychological and chemical risk items. Encounter data and NLP were used for the reliability item. Intractability and social support items were populated using NLP only. Information on oral morphine equivalents, length of treatment, changes in pain scores and NLP were used to populate the efficacy item. If no information was found, most items were scored as 3 and efficacy as 2 . The results of the NLP versus clinician-administered validation kappa analysis and test characteristics are pending.

Conclusion: Among major barriers to appropriate management of chronic noncancer pain with opioids are inadequate time and resources available to clinicians at a point of care for risk assessment. Novel approaches, such as NLP, may support clinical decision-making by automating the process of data extraction.

Keywords: natural language processing, risk assessment 
PS2-75:

\section{Automating Research Data Using Metadata- Driven Processes to Dynamically Create Loader Programs}

\section{Shannon Leitch, ${ }^{1}$ Alan E. Bauck, ${ }^{1}$ Mike Allison, ${ }^{1}$ Catherine Cleveland, ${ }^{1}$ Matt Hornbrook ${ }^{1}$ \\ ${ }^{1}$ Center for Health Research, Kaiser Permanente Northwest, Portland, OR}

Background/Aims: The growing complexity and heterogeneity of source data systems makes maintaining a research data warehouse challenging, especially with the increasing need for current data with shorter refresh cycles and consistent results. A stable extract-transformload (ETL) environment is essential to maintain consistent results. To address inconsistency, high maintenance costs and the need to rapidly adapt to data changes, Kaiser Permanente Northwest's Center for Health Research (CHR-NW) undertook an effort to automate the ETL loading process.

Methods: CHR-NW developed a dynamic loader program to generate, validate and execute on-demand programs that load data into the production version of the Virtual Data Warehouse. It was developed in SQL Server Integration Services, and SQL Server Agent is used for scheduling and execution. Transform programs add entries to a task table upon completion, which the dynamic loader uses to identify staging tables with new data and then generate a loader program. Programs are generated using metadata about the staging tables, allowing adaptation to changes in the data structures and rapid deployment of new content.

Results: The dynamic loader approach enables CHR-NW to generate loader code without manual intervention. Its use of metadata in the staging environment provides the mechanism to adapt to changes in the data structures. It has reduced the effort needed to maintain the load step of the ETL process by reducing the number of programs from 55 to 1 . As a reference, in the first half of 2015, those 55 loader programs were updated an average of four times each and each program change took approximately 2 hours to change, review and test. That effort has largely been eliminated. The load process also runs more consistently, reducing the error rate from 0.1 to 0.005 . Business logic and new and developing best practices can be applied in one place and are automatically implemented. In one recent example, CHRNW was able to reduce load times for large tables by $50 \%$ (34 minutes to 15 ).

Conclusion: Developing the dynamic loader has increased programmer productivity and the consistency of data load results, allowing CHR-NW to rapidly apply techniques to improve computing processing efficiency.

Keywords: Virtual Data Warehouse, dynamic programming
PS2-76:

Direction of Type 2 Diabetes Mellitus Progression

\author{
Era Kim, ${ }^{1}$ David S. Pieczkiewicz, ${ }^{2}$ Pedro J. Carballo, ${ }^{3}$
} Regina Castro, ${ }^{3}$ Gyorgy J. Simon ${ }^{3}$

\section{${ }^{1}$ Medica Research Institute, Minnetonka, MN; ${ }^{2}$ Institute for Health Informatics, University of Minnesota, Minneapolis, MN; ${ }^{3}$ Mayo Clinic, Rochester, MN}

Background/Aims: Due to the progressive nature of type 2 diabetes mellitus (T2DM), deterioration in a patient's general health is inevitable. Hence, the overall health of a T2DM patient is poor. Markers of worsening overall health such as metabolic abnormalities are predominant in the progression to any of the T2DM complications, which mask patterns of progression to individual complications, thereby making it difficult to predict which particular complication a patient will progress to (i.e. direction of progression). We aim to unmask the patterns and develop the most distinctive and predictive markers of progression to each individual complication.

Methods: We take a two-step modeling approach using 94,536 T2DM patients' 10 -year, de-identified data from the OptumLabs $^{\mathrm{TM}}$ Data Warehouse, a database that includes retrospective administrative claims data on commercially insured and Medicare Advantage enrollees, as well as electronic medical record data from a nationwide network of provider groups. First, we build a "general progression" model that predicts development of any new complication and captures markers of general health deterioration. Next, we develop "differential progression" models that predict progression to individual complications. We use least absolute shrinkage and selection operator (LASSO)-penalized Cox regressions to select variables and penalize the "differential progression" models against the "general progression" model such that "differential progression" patterns become unmasked. Significance of markers is determined via permutation test and predictive performance is evaluated through bootstrap estimation.

Results: For a 6-year follow-up period, 31,968 (34\%) of all patients progressed to at least one complication: chronic kidney disease $(12 \%)$, ischemic heart disease $(11 \%)$, cerebrovascular disease $(8 \%)$, congestive heart failure $(5 \%)$, peripheral vascular disease $(5 \%)$, and renal failure (1\%). In this ongoing research, we anticipate our predictive model to demonstrate improved interpretation of progression to each individual complication without causing reduced predictive performance.

Conclusion: We study the progression of T2DM to multiple complications systematically and comprehensively by applying data mining techniques on the large amount of claims and clinical data from the OptumLabs Data Warehouse. By unmasking previously masked patterns and developing markers that suggest progression to a specific target complication, our study findings will assist clinicians in the management and treatment of T2DM.

Keywords: data mining, predictive model, progression of type 2 diabetes mellitus

(C) 2016 Aurora Health Care, Inc. 